\title{
MICRO PROPULSION IN LIQUID BY OSCILLATING BUBBLES
}

\author{
by
}

\section{Jian Feng}

B.S. in Mechanical Engineering, University of Science and Technology, Beijing, 2006

M.S. in Mechanical Engineering, University of Science and Technology, Beijing, 2009

Submitted to the Graduate Faculty of

Swanson School of Engineering in partial fulfillment

of the requirements for the degree of

Doctor of Philosophy

University of Pittsburgh 
UNIVERSITY OF PITTSBURGH

SWANSON SCHOOL OF ENGINEERING

This dissertation was presented

by

Jian Feng

It was defended on

November 19, 2015

and approved by

Jeffrey Vipperman, PhD, Professor, Vice Chair of MEMS Faculty

Department of Mechanical Engineering and Materials Science

David Schmidt, PhD, Professor,

Department of Mechanical Engineering and Materials Science

Youngjae Chun, PhD, Assistant Professor,

Department of Industrial Engineering, Department of Bioengineering

Dissertation Director: Sung Kwon Cho, PhD, Associate Professor,

Department of Mechanical Engineering and Materials Science 
Copyright (C) by Jian Feng

2016 


\title{
MICRO PROPULSION IN LIQUID BY OSCILLATING BUBBLES
}

\author{
Jian Feng, PhD \\ University of Pittsburgh, 2016
}

A number of attempts have been made to fabricate microswimmers that possibly navigate in vivo including the artificial magnetic bacteria flagella, chemical microswimmers and natural organism based microswimmers. This paper presents another propelling mechanism in micron scale that works by oscillating microbubbles in acoustic field.

First of all, the propulsion mechanism is proven by two-dimensional computational fluid dynamics (CFD) simulations. Then, the microswimmer device is made on a parylene structure by photolithography. The underwater propulsion in one-dimensional is demonstrated and the propulsion mechanism is also confirmed by experiments. The relation of the propulsion speed/bubble oscillation amplitude and the input acoustic signal is measured. It is shown that the propulsion will happen when the bubble oscillation amplitude (or Reynolds number) gets large enough which is close to the system acoustic resonance. Around this resonance frequency (about $11 \mathrm{kHz}$ ), the measured propulsion speed is up to $45 \mathrm{~mm} / \mathrm{s}$ and payload-carrying ability is realized. The one-directional rotation acoustic turbo is also made with a speed of about $75 \mathrm{rpm}$. This acoustic frequency dependence also becomes the foundation for two-dimensional propulsion.

Then, the bi-directional motion and two-dimensional steering motion are realized by microbubbles with different lengths based on their different acoustic resonances. First of all, the frequency behavior for long (about $760 \mu m$ average length) and short (about $300 \mu m$ average length) bubbles at about $6 \mathrm{kHz}$ and $11 \mathrm{kHz}$ are measured, including oscillation amplitude and 
generated microstreaming. By adjusting input acoustic frequency, specific bubbles could be activated selectively. Then, when the different microbubbles are arranged into opposite directions, the bi-directional propulsion can be realized, including back/forth motion and clockwise/counter-clockwise rotation. The bi-directional motion mechanism is also confirmed by three-dimensional CFD simulations and the net force is calculated. The concept is further developed into two-dimensional propulsion by arranging long and short bubbles into orthogonal directions on the same device. By switching the input acoustic frequency, the controlled steering propulsion is illustrated on a two-dimensional plane. Carrying of objects in a T-junction microchannel is shown as well.

The last part of this thesis is focused on developing the microswimmer into a biodegradable device, including long- and short-tem. The long-term biodegradable device is fabricated by polycaprolactone (PCL) by a simple dipping method, and propulsion in a minitube is shown. The short-term biodegradable device is fabricated by rolling up magnesium film based on building stress mismatch mechanically with help of a stretcher. The method could also be applied to aluminium and parylene film rollups. At last, the propulsion and biodegradable abilities of magnesium microtube are demonstrated. 
TABLE OF CONTENTS

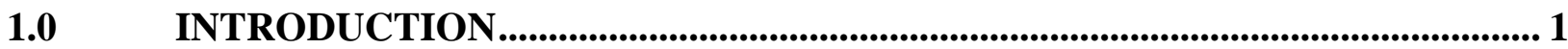

1.1 BACKGROUND ..................................................................................................... 1

1.2 PROPULSION IN MILLIMETER SCALE _............................................... 3

1.3 PROPULSION IN MICRON AND NANOMETER SCALE ........................... 3

1.3.1 Propulsion by Irreversible Strokes ................................................................... 6

1.3.2 Propulsion by Bubbles........................................................................................... 7

1.3.3 Propulsion by Magnetic Field................................................................................. 7

1.3.4 Propulsion by Other Mechanisms............................................................................ 11

1.4 CONCLUDING REMARKS AND CHALLENGES....................................... 13

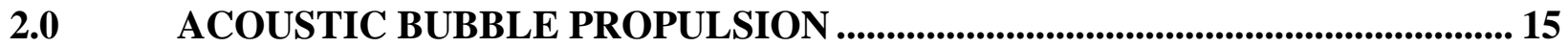

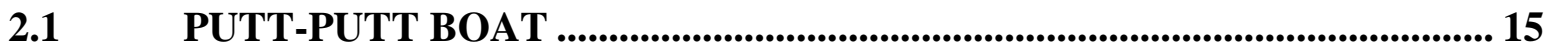

$2.2 \quad$ ACOUSTIC BUBBLE MILLIMERTER SIZE ................................................ 17

2.3 ONE-DIMENSIONAL MICRON SCALE ACOUSTIC BUBBLE

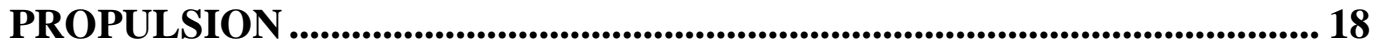

2.3.1 Working Principle and Computational Fluid Dynamics Analysis............. 19

2.3.2 Device Fabrication and Experimental Set-up .................................................... 31

2.3.2.1 MICROFABRICATION........................................................................ 31

2.3.2.2 EXPERIMENTAL SET-UP.................................................................... 37 
2.3.3 Experimental Results ..................................................................................... 39

2.4 BI-DIRECTIONAL AND TWO-DIMENSIONAL MICRON SCALE

ACOUSTIC BUBBLE PROPULSION................................................................. 51

2.4.1 Device Fabrication and Experimental Set-up ............................................ 53

2.4.2 Acoustic Measurement ..............................................................................54

2.4.2.1 EFFECT OF WATER LEVEL.............................................................. 54

2.4.2.2 EFFECT OF BUBBLE LENGTH.................................................... 56

2.4.3 Bi-directional Propulsion ........................................................................... 58

2.4.4 Two-dimensional Propulsion ............................................................................. 66

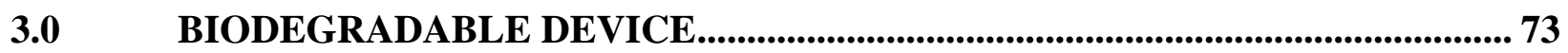

3.1 BIODEGRADABLE DEVICE FABRICATION........................................... 73

3.1.1 Long-term Biodegradable Device.......................................................................... 74

3.1.2 Short-term Biodegradable Device ..................................................................... 83

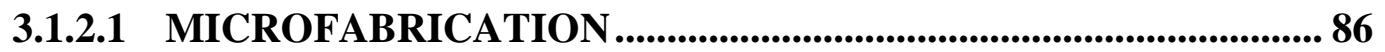

3.1.2.2 FABRICATION RESULTS ............................................................ 89

3.1.2.3 ACOUSTIC BUBBLE PROPULSION AND BIODEGRADATION

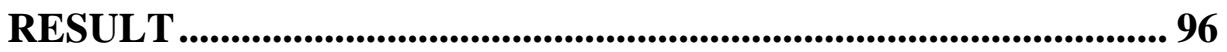

4.0 CONCLUSION REMARKS …….......................................................................... 101

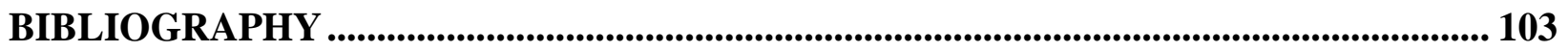




\section{LIST OF TABLES}

Table 1. Acoustic pressure measurement by hydrophone at different input voltage for $11.2 \mathrm{kHz}$

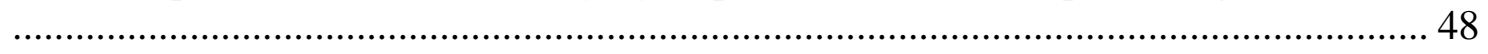

Table 2. Oscillation amplitude for six short and three long bubbles at $5.80 \mathrm{kHz}$ and $11.2 \mathrm{kHz} . .60$

Table 3. Property and price for biodegradable polymers...................................................... 75

Table 4. Physical properties of PCL and parylene C.......................................................... 76

Table 5. The summary of the pathophysiology and toxicology of $\mathrm{Mg}$ and some alloying elements

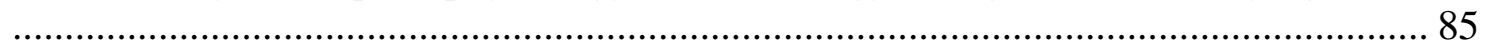




\section{LIST OF FIGURES}

Figure 1.1. Snapshot in the movie (1966) "Fantastic Voyage” (miniaturized shuttle and crew navigate the human body).

Figure 1.2. Propulsion speed and Reynolds number for artificial and natural microswimmers. 1acoustic scallop ( $R e$ is based on oscillating speed and amplitude) [17], 1'-acoustic scallop ( $R e$ is based on body speed and size), 2-oscillating micro bubble ( $R e$ is based on oscillating speed and amplitude) [18], 2'-oscillating micro bubble ( $R e$ is based on body speed and size), 3-artificial magnetic bacteria flagella [19], 4-artificial magnetic nanostructured propeller [20], 5-magnetically actuated colloidal [21], 6magnetotactic bacteria propeller [22], 7-flagella-based propulsion [23], 8-active Brownian Janus [33], 9-self-motile colloidal [25], 10-catalytic nanomotor by bubble [26], 11-molecular motor [27], 12-striped metallic nanorod [30], 13-catalytic microtubular jet [28], 14-Pt-Au-Ppy motor with $1.05 \mu \mathrm{m}$ radius cargo [31], 15$\mathrm{Au} / \mathrm{Ni} / \mathrm{Au} / \mathrm{Pt}-\mathrm{CNT}$ motor with $4.3 \mu \mathrm{m}$ diameter cargo [32], 16-Zinc microrockets [29], 17-paramecium [15], 18-human spermatozoon [15], and 19-Escherichia coli [16]. Note that the triangles denote inertia dominant propulsion, the squares denote viscous dominant propulsion, and the circles means the propulsion mechanisms cannot be clearly classified.

Figure 1.3. (a) Schematic of a DNA linked flexible magnetic filament [54]; (b) SEM image of ABF with diameter of $2.8 \mu m$ [21].

Figure 1.4. (a) Schematic of the cDNA strands linked colloidal doublets motion in an external magnetic field [23]. (b) Schematic of the squared magnetic micro robot [63]........ 10

Figure 2.1. The flow field obtained from particle tracking velocimetry (PTV) at two phases of the acoustic cycle (a) phase $144^{\circ}$ (intake flow); (b) phase $216^{\circ}$ (outtake flow) [19].17

Figure 2.2. Concept of propulsion by oscillating bubble columns. Note that orthogonal arrangement of the bubbles can generate steering motion. The inset shows the fabricated single bubble device.

Figure 2.3. Computational domain of the bubble propulsion device placed and centered between two walls. Only the right half of the full domain is simulated under the assumption of flow symmetry between the left and right halves. 22 
Figure 2.4. Numerical Results of streamlines for both discharge and intake cases at different Reynolds number (a) $R e=0.38$, discharge, (b) $R e=0.38$, intake, (c) $R e=380$, discharge and (d) $R e=380$, intake. 25

Figure 2.5. Numerical Results of (a) propulsion forces and (b) non-dimensionalized propulsion force at different Reynolds number.

Figure 2.6. Numerical Results of streamlines and velocity magnitude for both (a) intake and (b) discharge cases at five different Reynolds numbers $R e=380, R e=38, R e=3.8$, $R e=0.38$ and $R e=0.038$. 29

Figure 2.7. (a) Fabrication of one-end open microchannel and (b) photo of fabricated device (top view and frontal view at the channel opening). The microchannel traps and holds an air bubble column. One end of the channel is opened by RIE etching. 32

Figure 2.8. Fabrication steps (a) two masks design, (b) after first and second parylene coating and sacrificial photoresist pattern, (c) after Al mask sputtering, (d) after second photoresist pattern, (e) after RIE etching, (f) a close view of the open end of microchannel, (g) Al mask etched by acetone and (h) sacrificial photoresist etched by acetone. 36

Figure 2.9. Bubble meniscus formed in one oscillation cycle (a) convex and (b) concave meniscus. 39

Figure 2.10. Flow visualization of streaming flow. The particles are pushed away from the cylinder open-end when the bubble is oscillating. 41

Figure 2.11. Generation of streaming flow. After one min. bubble oscillation, seeding particles are pushed away from the cylinder open-end, as the empty region is distinctively seen. Applied voltages to piezo-actuator are (a) 77 (b) 126 and (c) $165 V_{p p} \ldots \ldots . . . . . .42$

Figure 2.12. Propulsion verification: two consecutive snapshots for different orientations of the cylinder with respect to the testing tank; (a) and (b) moving up. (c) and (d) moving down. (e) and (f) moving left. (g) and (h) moving right.

Figure 2.13. (a) Propulsion by a single bubble $\left(11.2 \mathrm{kHz}, 170 V_{p p}\right.$; see supplementary video clip 2, 60x slower; Insets show bubble menisci and channel opening), (b) Propulsion by an array of bubbles (11.2 kHz, $170 V_{p p}$; see supplementary video clip 3, 16x slower), (c) Rotation by bubbles embedded on turbo blades (3.8 kHz, $480 V_{p p}$; see supplementary video clip 4, 1.1x faster) and (d) Payload carrying by an array of bubbles $\left(11.2 \mathrm{kHz}, 190 \mathrm{~V}_{p p}\right.$; see supplementary video 5, 60x slower for translation and $25 x$ slower for rotation). The inset shows a side view of the arrayed bubbles and load. Note that due to aliasing of image sampling the bubble oscillations look slower than the applied frequency.

Figure 2.14. An array of oscillating bubbles carries a load of several millimeters in size: (a) initial configuration; and (b) transporting and rotating of the load by the bubble array. 45 
Figure 2.15. Propulsion speed vs. (a) applied voltage and (b) frequency. 47

Figure 2.16. Input voltage vs. acoustic pressure inside the testing water tank....................... 49

Figure 2.17. Input frequency vs. acoustic pressure inside the testing water tank...................... 50

Figure 2.18. Oscillation velocity of the tank bottom at different frequency with different water

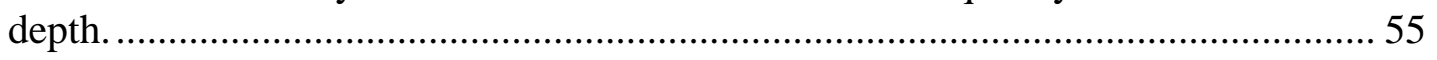

Figure 2.19. Streaming speed vs. frequency for $820 \mu m$ and $270 \mu m$ long bubbles at $150 V_{p p . . . .57}$

Figure 2.20. Bi-directional (back and forth) propulsion (bottom view) by (a) $820 \mu m$ long bubbles at $5.8 \mathrm{kHz}$ and (b) $270 \mu \mathrm{m}$ short bubbles at $11.2 \mathrm{kHz}$.............................. 59

Figure 2.21. Simulated streamlines of the microchannel when placed (a) on the bottom of channel and intakes, (b) on the bottom of channel and discharges, (c) in the middle of the channel and intakes and (d) in the middle of the channel and discharges. ..... 62

Figure 2.22. Numerical results of the propulsion force for all the short and long bubbles at (a) 5.8 $\mathrm{kHz}$ and (b) $11.2 \mathrm{kHz}$. 63

Figure 2.23. Rotation (bottom view) (a) clockwise rotation by $270 \mu \mathrm{m}$ long bubbles at $11.0 \mathrm{kHz}$, (b) counterclockwise rotation by $820 \mu \mathrm{m}$ bubbles at $5.8 \mathrm{kHz}$ and (c) bubble turbomotor at $3.8 \mathrm{kHz}$. 66

Figure 2.24. Two-dimensional motion device with three $820 \mu m$ and three $270 \mu m$ bubbles...... 67

Figure 2.25. 2-D motions (a) the long bubbles push, (b) the short bubbles push and (c) all the bubbles push under superposed acoustic field of the two frequencies (particles were seeded for flow visualization). 68

Figure 2.26. Steering capability in a T-junction of channel by $820 \mu \mathrm{m}$ and $270 \mu \mathrm{m}$ bubbles without tracking particles (a), (b) moving in (c), (d) counter-clockwise rotation and (e), (f) moving out. 69

Figure 2.27. Steering capability in a T-junction of channel by $820 \mu \mathrm{m}$ and $270 \mu \mathrm{m}$ bubbles with tracking particles (a), (b) moving in (c), (d) clockwise rotation and (e), (f) moving out. 70

Figure 2.28. Steering capability in a T-junction of channel by $820 \mu \mathrm{m}$ and $270 \mu \mathrm{m}$ bubbles with load with (a) moving in, (b) switching frequency and (c) moving out. .................. 71

Figure 3.1. Fabrication process of one-end open PCL microchannel.................................... 79

Figure 3.2. PCL microswimmer propulsion in the glass pipe........................................... 81

Figure 3.3. Microtube (a) fabrication concept, (b) strain generation between two layers by mechanical stretcher and (c) fabrication process flow. 86 
Figure 3.4. Magnesium film rollup (a) before acetone etching of sacrificial Pr, (b) during acetone etching, (c) without pre-strain in the $1^{\text {st }}$ layer, (d) $1200 \mu \varepsilon$ pre-strain in the $1^{\text {st }}$ layer, (e) $2000 \mu \varepsilon$ pre-strain in the $1^{\text {st }}$ layer and (f) $3800 \mu \varepsilon$ pre-strain in the $1^{\text {st }}$ layer..... 91

Figure 3.5. Aluminium film rollup (a) $1100 \mu \varepsilon$ pre-strain and (b) $1900 \mu \varepsilon$ pre-strain in the 1st layer. Parylene film rollup (c) $1800 \mu \varepsilon$ pre-strain and (d) $3200 \mu \varepsilon$ pre-strain in the 1st layer.

Figure 3.6. $130 \mathrm{~nm}$ thick aluminium layer detached at $4000 \mu \varepsilon$ (a) overall view of the pops up straight lines pattern and (b) an enlarged view.

Figure 3.7. Microtube radius vs. pre-strain for magnesium, aluminium and parylene film with curve-fits by Eq. (14). 96

Figure 3.8. (a) Aluminium microtube dried in air, (b) trapped bubble oscillation in water (particle seeded for flow visualization) and magnesium microtube (c) start of propelling motion, (d) end of propelling motion by acoustic bubble oscillation (11.0 kHz, 175 $\left.V_{p p}\right)$. 98

Figure 3.9. Magnesium microtube degraded in tap water at (a) initial time and (b) after 1 day. 99 


\subsection{INTRODUCTION}

\subsection{BACKGROUND}

In the near future, a dream technology for medical science and biology may become reality, as shown in the classic movie, "Fantastic Voyage" (Figure 1.1), in which a crew driving a miniaturized submarine navigates through blood streams to cure the abnormal areas in the human body. To realize this fantastic technique, a lot of scientists and engineers have been stimulated and encouraged to explore and make such a medical microswimmer. Due to the advent of micro/nanofabrication technology and highly concerted inter/multi-disciplinary research activities, the realization of this kind of robot may not be just science fiction anymore, but may possibly bring about a revolution in medicine in the near future $\mathrm{e}^{1-3}$. In developing such swimming medical robots, there are currently two major challenges: (1) lack of an efficient propelling and steering mechanism and (2) powering the robots. Thus far, numerous attempts have been made to tackle these challenges.

Generally speaking, there are two major physical mechanisms that generate a propulsion force on a small scale to overcome the drag force exerted by the surrounding liquid: inertia propulsion and viscous propulsion ${ }^{4}$. The inertia propulsion generates displacement of mass to exchange momentum between the swimming body and the surrounding fluid. The viscous propulsion uses viscous stresses with the surrounding fluid to generate a propulsion force. To classify these two 
propulsion mechanisms, the Reynolds number $(R e)$, which stands for the ratio between the inertia force and the viscosity force, is commonly used. The Reynolds number is defined by $R e=U L / v$, where $U$ is the characteristic velocity (the propulsion speed or actuator speed), $L$ is the characteristic physical dimension (swimming body size or actuator size), and $v$ is the kinematic viscosity of the fluid. For a large $R e$, the inertia force is dominantly responsible for propulsion while for a small $R e$, the viscous force is responsible. As the physical dimension critically affects the Reynolds number, a suitable propulsion mechanism should be selected and used based on the size of the propulsion object or actuator.

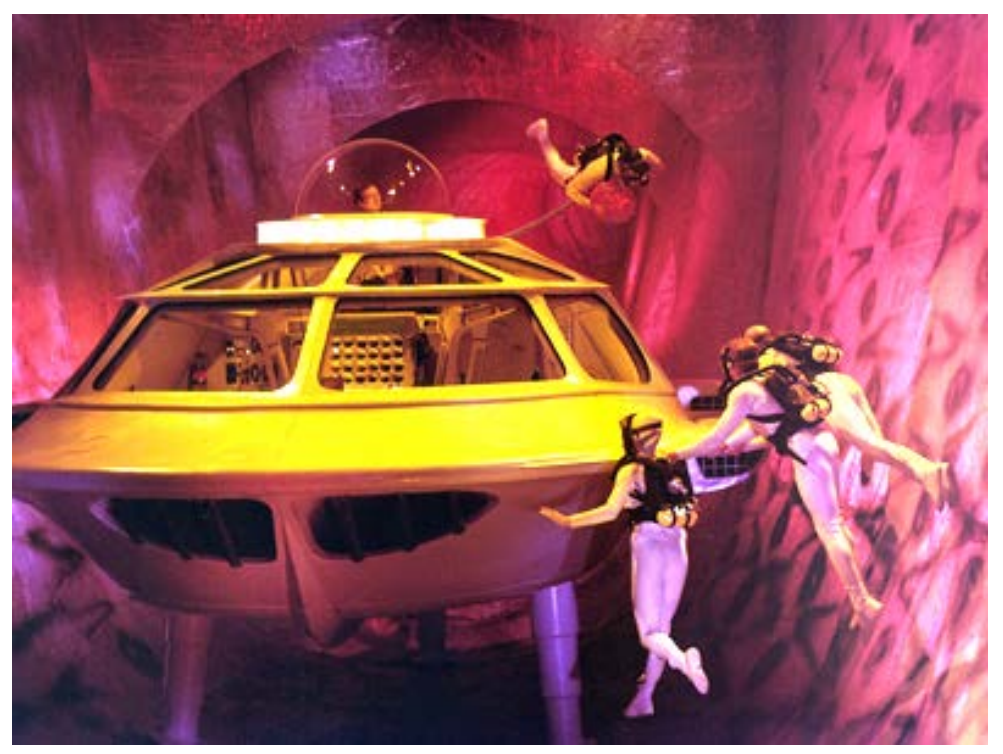

Figure 1.1. Snapshot in the movie (1966) "Fantastic Voyage” (miniaturized shuttle and crew navigate the human body). 


\subsection{PROPULSION IN MILLIMETER SCALE}

Propulsion over the size of millimeters may be too big to fit in the medical robots swimming inside of the human body. However, it still gives us some basic idea of the propulsion mechanism. Most of them are made by smart materials to realize propulsion in a fluidic environment, such as by using shape memory alloy (SMA $)^{5-7}$, ionic conducting polymer film $(\mathrm{ICPF})^{8-11}$ and piezoelectric actuators ${ }^{12,13}$. For the above macro-scale swimmers, the estimated Reynolds number are over $10^{2}$, which means the propulsion force is based on the inertia effect: The viscous effect is negligible.

This thesis is focused on the propulsion on an even smaller scale, therefore past and current research works based on different propelling principles of the dimensions ranging from millimeters to nanometers will be introduced in the next section 1.3. Here, $R e$ is used to characterize propulsion principles.

\subsection{PROPULSION IN MICRON AND NANOMETER SCALE}

When the dimension of swimming vehicles goes into the micron/nanometer scale, the propulsion principle is quite different from that of the larger scale. In addition, swimming in such a small scale faces a lot of challenges, including spatial and temporal control of the swimming direction and speed ${ }^{14}$. In order to compare a variety of propulsion methods in this scale, $R e$ and propulsion speed are plotted in Figure 1.2. For reference, the data for some natural microorganisms are added (paramecium $\sim 100 \mu \mathrm{m}$ long, human spermatozoon $\sim 50 \mu \mathrm{m}$ long and Escherichia coli $\sim 3 \mu \mathrm{m}$ long) ${ }^{15,16}$. Some of the fabricated micro devices could swim in the low 
Reynolds number region (even less than $10^{-5}$ ) that is much smaller than the natural swimmers. In general, however, the natural microorganisms have a relatively faster speed ( 10 body lengths/second) than the artificial devices. Currently, almost all the developed propulsion devices cannot generate a force strong enough to propel against strong blood streams such as in the aorta, but they can be operated in quiescent or mild flow regions. On the other hand, some swimmers that have a fast speed, for example, device 2 in Figure 1.2, cannot swim in a very low Reynolds number. The direction of future research (i.e., faster propulsion and smaller swimmer) is expected to turn toward the upper-left corner in the Figure 1.2 (indicated by the arrow).

For almost all of the swimming robots in the millimeter scale, the propulsion mechanisms are based on inertia propulsion. However, for the swimming robots in the micron/nanometer scale, both inertia propulsion and viscous propulsion are possible, depending on the types of devices. From the authors' perspective, the developed propulsion mechanisms are classified as shown in Figure 1.2. The triangles in Figure 1.2 denote inertia-dominant propulsion; the squares denote viscous-dominant propulsion; and the circles means the mechanisms are not clear. In Figure 1.2, the inertia propulsion methods are located in the large Re region, while the viscous propulsion methods are in the low Re region. More specifically, the bubble type (putt-putt boat type) swimmers are using momentum transfer through the micro streaming formed by bubble oscillation $^{17,18}$. The micro robots that harness natural organisms or use the artificial cilia/flagella (regardless of motion types, corkscrew motion, or flexible oar motion) generate propulsion via viscous stress interaction ${ }^{15,16,19-24}$. Among the chemical microswimmers, even though there are still debates on the mechanism ${ }^{25}$, some devices utilize the bubble recoiling method to make momentum transfer by inertia propulsion ${ }^{26-29}$. The electric and thermophoresis methods may be categorized as viscous propulsion, as the active Brownian motion method ${ }^{30-33}$. 


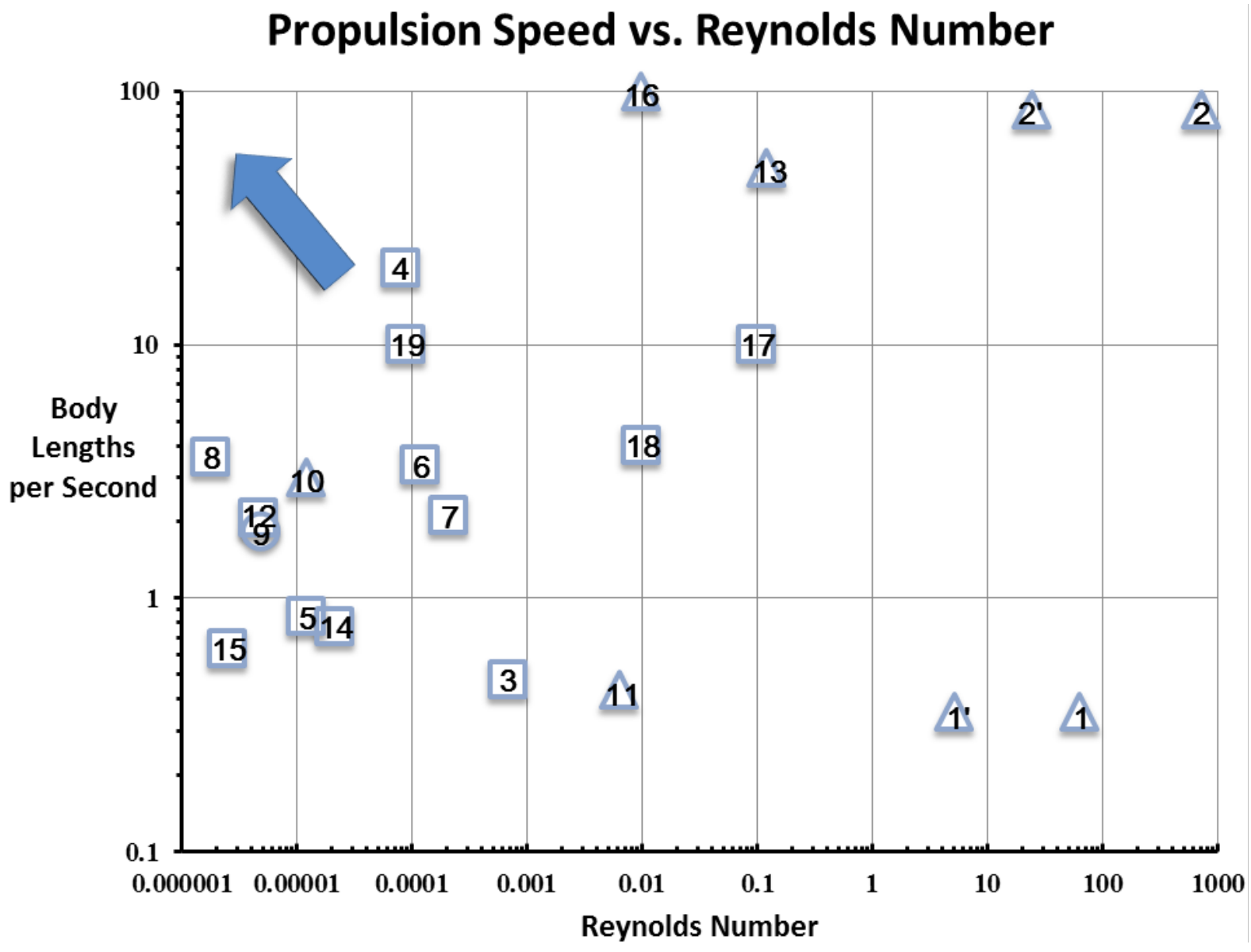

Figure 1.2. Propulsion speed and Reynolds number for artificial and natural microswimmers. 1-acoustic scallop (Re is based on oscillating speed and amplitude) [17], 1'-acoustic scallop (Re is based on body speed and size), 2-oscillating micro bubble (Re is based on oscillating speed and amplitude) [18], 2'-oscillating micro bubble (Re is based on body speed and size), 3-artificial magnetic bacteria flagella [19], 4-artificial magnetic nanostructured propeller [20], 5-magnetically actuated colloidal [21], 6-magnetotactic bacteria propeller [22], 7-flagella-based propulsion [23], 8-active Brownian Janus [33], 9-self-motile colloidal [25], 10-catalytic nanomotor by bubble [26], 11-molecular motor [27], 12-striped metallic nanorod [30], 13-catalytic microtubular jet [28], 14-Pt-Au-Ppy motor with $1.05 \mu m$ radius cargo [31], 15-Au/Ni/Au/Pt-CNT motor with $4.3 \mu m$ diameter cargo [32], 16-Zinc microrockets [29], 17-paramecium [15], 18-human spermatozoon [15], and 19-Escherichia coli [16]. Note that the triangles denote inertia dominant propulsion, the squares denote viscous dominant propulsion, and the circles means the propulsion mechanisms cannot be clearly classified. 


\subsubsection{Propulsion by Irreversible Strokes}

If the Reynolds number is very low, the fluid motion can be easily reversed by simply reversing the stroke of the actuator. In other words, the motion created in one degree of freedom is always reciprocal (reversible), as stated in the scallop theorem ${ }^{34}$. Therefore, to generate propulsion in the low $R e$, making an irreversible motion is needed, for example a flexible oar motion and a corkscrew motion ${ }^{34}$ that mimic those of many bacteria. There is a comparison between these two swimming modes showing that they have very comparable peak performance $^{35}$.

In addition to these two methods, there are also other propulsion principles for micro robots that would work for the low Re, for example, the Purcell's three-link swimmer ${ }^{34}$. Becker et al. ${ }^{36}$ made a detailed numerical study on this model. The results showed that this robot could swim in both directions along the straight line. However, the optimal three-link swimmer still had much lower efficiency compared to with the flexible oar motion or the corkscrew motion. Najafi et $a l .{ }^{37}$ proposed another model with three linearly linked three spheres for a micro robot that is operated based on the periodical internal motion. According to the numerical study, this

swimmer could also work in the low Re range. Leoni et $a .^{38}$ first realized this model experimentally with optical tweezers and compared the experimental results with the numerical solution. All of these research works have made a great contribution to mini/micro underwater swimmer propulsion. 


\subsubsection{Propulsion by Bubbles}

An oscillating bubble system could also be used to realize the propulsion due to increasing the frequency of the motion (speed of the induced flow), therefore increasing the $R e$. It is well known that when a gaseous bubble is periodically excited by an acoustic or temperature field, the bubble shrinks and expands as the oscillating wave of the pressure or temperature passes the bubble. Here, the high frequency oscillation generates a unidirectional, non-zero time-averaged velocity component, which is called microstreaming ${ }^{39}$. The microstreaming flows have been applied to micropumps or micromixers ${ }^{40,41}$.

There are different ways to actuate the bubble and generate the microstreaming, such as by using AC-electro wetting which could oscillate bubbles (300 $\mu \mathrm{m}$ and $1.5 \mathrm{~mm}$ diameter) to generate a steady streaming flow for propulsion ${ }^{42}$. The bubble oscillation was made by electrically changing the contact angle of the bubbles ${ }^{43}$. In addition, the thermal excitation could also be used for the similar propulsion ${ }^{44}$ where the oscillation frequency is quite low $(100 \mathrm{~Hz})$. In this thesis, more research is done by creating the acoustically actuated oscillating bubbles which are going to be introduced in section 2.0 .

\subsubsection{Propulsion by Magnetic Field}

The non-contact magnetic field actuation also draws a lot of attention to micro robot propulsion with a possible application to in vivo drug delivery, nonsurgical exploration, and navigation ${ }^{45}$. For instance, a spiral-type micro robot was designed by Ishiyama et al. ${ }^{46}$, which mimicked the cilium propulsion of natural bacteria. The robot could even swim in high viscosity oil $\left(500 \mathrm{~mm}^{2} / \mathrm{s}\right)$ at $R e$ of about $10^{-7}$ and had a maximum velocity of $0.022 \mathrm{~mm} / \mathrm{s}$. Recently, the 
work has been extended to a smaller scale and lower Re. Similar to the "flexible oar motion" as mentioned above, Dreyfus et al. ${ }^{47}$ developed a flexible artificial flagellum that was propelled by a propagating magnetic wave. The robot was made of red blood cells linearly chained by the magnetic colloids, $1 \mu \mathrm{m}$ in diameter and several DNA molecules of $107 \mathrm{~nm}$ in length, as shown in Figure 1.3 (a). When the homogenous and sinusoidal external magnetic fields were applied, a bending wave was generated in the filament, and the motion was non-reversible. Depending on the ratio between the tangential and perpendicular viscous coefficients, the filament swam towards the free end of the filament ${ }^{48}$. Williams et $a l^{24}$ also made this type of microswimmer which was self-propelled. By the standard photolithography and dry etching process, polydimethylsiloxane (PDMS) filaments were made and attached to contractile cells. As the cells were selectively seeded on a single region of the filament, time-irreversible deformations were generated to produce the propulsion force.

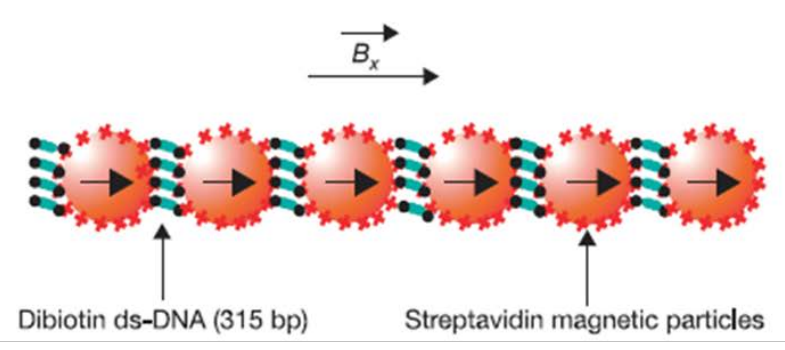

(a)

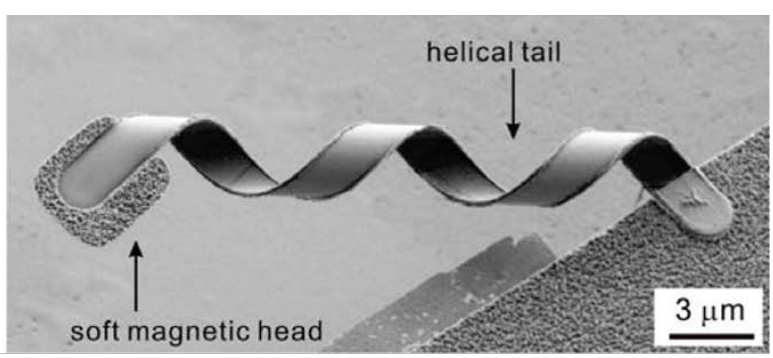

(b)

Figure 1.3. (a) Schematic of a DNA linked flexible magnetic filament [54]; (b) SEM image of ABF with diameter of $2.8 \mu m$ [21].

Another popular micro robot for the low Re in magnetic field is using the corkscrew motion. A numerical model has been set up for this type of swimmer which gave the relation among the 
swimming velocity, efficiency and external torque ${ }^{49,50}$. An artificial bacteria flagella $(A B F)^{51,52}$ was developed with a square soft-magnetic metal plate head and a helical tail using the anomalous scrolling process, as shown in Figure $1.3(\mathrm{~b})^{53}$. The ABF with a $38 \mu \mathrm{m}$ tail could swim forward and backward at the average speed of around $1.2 \mu \mathrm{m} / \mathrm{s}$ by changing the rotational direction of the external magnetic field. The propulsion force of the $29 \mu \mathrm{m}$ long ABF, which was estimated from the drag force of a single microsphere pushed by the ABF swimmer ${ }^{51}$, was about $0.17 p N$. Later, the quantitative analyze and mathematic model was done for this 6-degrees-offreedom motion in comparison with the experiments. The maximum velocity of ABF reached about $18 \mu \mathrm{m} / \mathrm{s}$, and the propulsion force was calculated to be about $3.0 p N^{19}$. For similar swimming robots but several millimeters in size, more experiments in both water and glycerin were performed to compare the inertia and viscous forces ${ }^{54}$. Ghosh et al. ${ }^{20}$ reduced the size of this magnetic propeller down to $200 \sim 300 \mathrm{~nm}$ wide and $1 \sim 2 \mu \mathrm{m}$ long. The swimmer had a high propelling speed in water ( $40 \mu \mathrm{m} / \mathrm{s})$, which was much faster than the Brownian motion. Moreover, it showed good steering ability. Based on a similar idea, Gao et al. ${ }^{55}$ simplified the fabrication process by the template electro deposition protocol. The Au "head" and Ni "tail" were connected by a flexible sliver joint, which was dissolved in a peroxide solution. By changing the size of "head" and "tail", this device could move either forward or backward in a rotational magnetic field. Because both head and tail could generate "cone surfaces" during rotation, and the amplitude was related with the size of the tail and head, the forces generated by both "head" and "tail" were proportional to the projection of the cone surface area in the direction of motion. 
In addition to the above propulsions, Tierno et al. ${ }^{21}$ made another propulsion through nonreversible motions using the colloids doublet which had two spheres of $1.0 \mu \mathrm{m}$ and $2.8 \mu \mathrm{m}$ in diameter (Figure 1.4). The cDNA strands linked these two spheres. When the magnetic field was applied, the smaller sphere could rotate around the mass center in $(x, z)$ plane and generate a viscous friction gradient in the $y$ direction by the boundary plate, as shown in Figure 1.4 (a). As a result, a motion in the $x$-direction was generated (up to $3.2 \mu \mathrm{m} / \mathrm{s}$ ). Floyd et $a l .{ }^{56}$ demonstrated a micro-machined square magnetic robot $\left(250 \times 130 \times 100 \mu m^{3}\right)$ which pushed a $116 \mu m$ diameter polystyrene bead in a water droplet under magnetic control, as shown in Figure 1.4 (b).

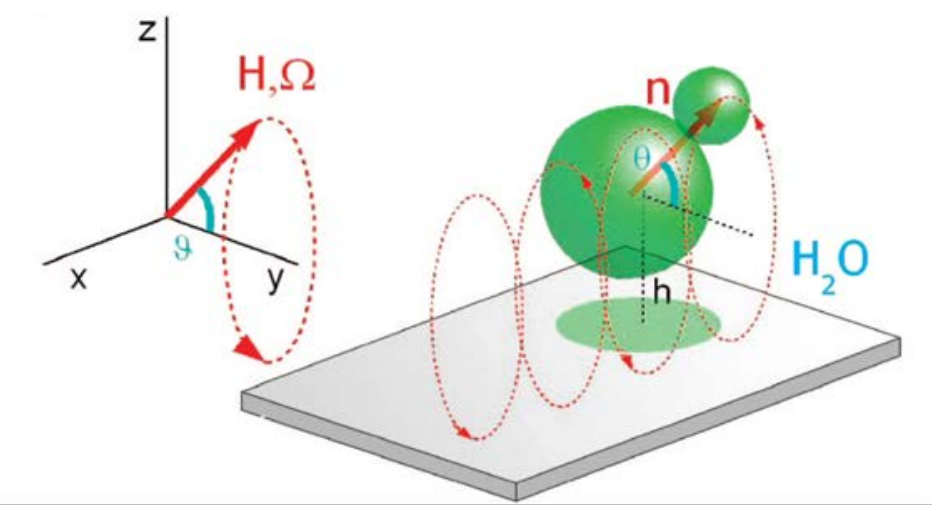

(a)

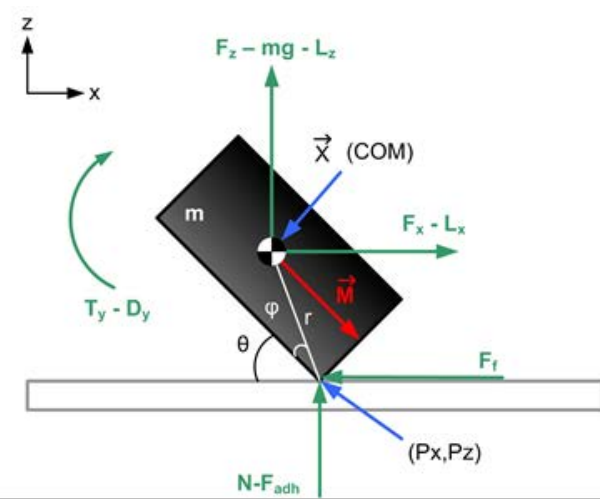

(b)

Figure 1.4. (a) Schematic of the cDNA strands linked colloidal doublets motion in an external magnetic field [23]. (b) Schematic of the squared magnetic micro robot [63]. 


\subsubsection{Propulsion by Other Mechanisms}

The above mechanisms for device propulsion are mainly about mechanics. Besides these, there are other propulsion mechanisms, such as biological propulsion and chemical propulsion that will be briefly introduced in this section.

Lee et al. demonstrated the assembly and transportation of magnetic nanoparticles by controlling the Magnetospirillum magnetotacticum ${ }^{57}$. Martel et al. ${ }^{22}$ used the Magnetospirillum gryphiswaldense magnetotactic bacteria (MTB) to push a $3 \mu \mathrm{m}$ diameter bead and demonstrated propulsion at an average speed of $7.5 \mu \mathrm{m} / \mathrm{s}$ with a controllable direction. Behkam et al. ${ }^{23}$ attached several S. marcescens bacteria to a $10 \mu \mathrm{m}$ polystyrene sphere to make a swimming micro robot. Hiratsuka et al. ${ }^{58}$ made a micro-size motor powered by bacteria that rotated at $1.5 \sim 2.6 \mathrm{rpm}$. Another biological propulsion was made by motor proteins that could move on a certain surface and could be powered by the hydrolysis of adenosine triphosphate (ATP). Kinesin/microtubules are a typical structure to generate this type of motion, such as the work done by Tarhan et al. ${ }^{59}$ and Fujimoto et al. ${ }^{60}$.

The active Brownian motion could also be the propulsion mechanism which breaks the thermal equilibrium resulting in the particle motion or transportation in a controlled direction ${ }^{61}$. Volpe et al. ${ }^{62}$ made Janus particles and studied the swimming behavior of the Janus particles in the patterned surroundings and demonstrated steering capability with arranged obstacles. Buttinoni et al. ${ }^{33}$ continued this study and showed good direction control by changing the intensity of a green laser beam, which was used to generate temperature difference in the Janus particle.

Self-thermophoresis is a self-phoretic motion that is caused by an imbalanced osmotic effect in a solid-liquid interface. It could also be used for the micro particle propulsion. The propulsion speed for the self-thermophoresis is a function of temperature gradient on the particle surface ${ }^{63}$. 
As the Janus particle has a temperature difference by absorbing laser light, a thermal slip flow would be generated. Jiang et al. ${ }^{64}$ first measured this flow and controlled the particle motion by adjusting the laser power.

Howse et $a l^{25}$ coated half of a polystyrene sphere with platinum, which was propelled by breaking hydrogen peroxide into oxygen and water. However, in a long time period, the propelling motion was random. Vicario et al. ${ }^{27}$ used a molecular approach to demonstrate a nanoswimmer composed of the object (micro particle), the tether, and the catalyst motor. The swimmer used hydrogen peroxide to produce oxygen bubbles and converted chemical energy into mechanical energy. Kline et al. ${ }^{30}$ made a rod-like robot which also used hydrogen peroxide for propulsion fuel and platinum/gold for the catalyst. In addition, a nickel segment was used for the direction control by magnetic field.

Solovev et al. ${ }^{28}$ developed a micro tubular jet using a multi-metallic film. The film had a platinum inner layer used for decomposing hydrogen peroxide and a ferromagnetic layer for magnetic direction control. The robot had a larger opening to which the oxygen bubbles were driven and moved by the Laplace pressure difference. The hydrogen peroxide fuel was provided to the smaller opening end. As a result, a continuous jet flow and thrust were generated.

Sundararajan et al. ${ }^{31}$ showed a catalytic motor based on the hydrogen peroxide fuel by using electrophoresis for the colloidal cargo attachment and trasportation in a magnetic field. The magnetic field was used for direction control. Burdick et $a l .{ }^{32}$ also demonstrated the propulsion of similar robots in microchannel networks. Another chemical reaction propulsion is by using polyaniline (PANI)/zinc in a strong acid solution to produce hydrogen bubbles ${ }^{29}$. Bipolar electrochemistry could be used for the metallic object swimming ${ }^{65}$. Through two redox reactions 
in opposite ends of zinc dendrite, an electro osmotic flow was generated in the presence of an electric field, thus, the dendrite was moved in the glass capillary.

Sumino et al. ${ }^{66}$ studied the propulsion of an oil droplet. The motion mechanism is based on the difference of surface tension induced by non-equilibrium chemical conditions. By changing the boundary conditions, the random motion generated by the inverted convective flow inside of the oil droplet could be controlled and turned into a periodic regular motion or rotation.

\subsection{CONCLUDING REMARKS AND CHALLENGES}

Different types of microswimmers are reviewed above and many of them have shown exciting performances and promises with the ability of speed/direction control. However, each approach still has its own challenges to be addressed.

Most of the smarter actuators including SMA and ICPF are still operating in the millimeter scale and are tethered to an external power. The magnetic propulsion shows a great swimming ability with six degrees of freedom. However, it needs several external bulky coils that are orthogonally aligned to precisely control the magnetic field. The bacterial propulsion needs to maintain the controlled environment for the bacteria to survive, and exactly controlling the direction in the active Brownian motion is still difficult. For chemical propulsion, chemical fuels that are not usually biologically compatible (and are even harmful in some cases) are required to be provided and consumed.

Compared with other propulsion methods, bubble propulsion has its own advantages. For example, it does not require a large external instrument as a power source like magnetic propulsion. Furthermore, it does not have to swim in a special solution like the chemical or 
bacteria propulsion. However, the previous study on bubble propulsion still has limitations. For instance, the AC-electro wetting method needs to be tethered. The size of devices and bubbles for thermal propulsion or acoustic propulsion is still confined to a relative large size, like millimeters. In all of the three bubble propulsion methods, acoustic propulsion is the most promising one to be realized in in vivo bio application due to the wireless power transportationthe power could be transferred by acoustic wave and easily generated by a simple piezoelectric actuator. Therefore, in this thesis, research work is done to make the acoustic powered bubble propulsion device that could swim in the micron scale, including one- and two-dimensional motion. Furthermore, to fulfill the in vivo missions, the microswimmer is fabricated of biodegradable material. 


\subsection{ACOUSTIC BUBBLE PROPULSION}

Most of the methods mentioned in the previous section 1.0 are operated based on irreversible motions. However, fast symmetric strokes still mean low $R e$. The key factor to get better performance in this actuation is to increase the frequency of the motion to create a relatively large Re flow near the actuators, for example, by using an actuator driven over several $\mathrm{kHz}^{4}$.

\subsection{PUTT-PUTT BOAT}

The concept for a 'putt-putt boat' begins with a patent application by Thomas Piot ${ }^{67}$ in 1891 in the UK for a boat with a small boiler and two exhaust tubes, later improved by McHugh. This kind of boat could realize propulsion without any moving parts. The working principle for this boat is that when heat is applied to the boiler, liquid water inside the boiler becomes steam. The steam is pushed out from the exhaust tubes into the surrounding water out of the boat, therefore the boat is propelled forward. Then the steam bubble is condensed, and the vacuum inside the boiler sucks the surrounding water back into the boiler. Therefore the boat moves backward a little bit which is less than the forward distance. In other words, in every cycle of water intake and discharge, the boat could have a net forward movement. As time goes by, a lot of intake and discharge cycles could be accumulated and make the boat swim forward. Nowadays, the 'puttputt boat' is even commercialized as a toy. 
One may have a question about the propulsion principle: Why the backward movement distance during water intake is smaller than the forward distance during water discharge? Dijkink et al. ${ }^{17}$ gives the explanation by boundary layer separation in his paper. When water comes into the exhaust tube, the pressure gradient is favorable and boundary layer along the tube stays attached. When water goes out from the exhaust tube, the boundary layer is separated due to the adverse pressure gradient. Therefore asymmetry is formed for water intake and discharge—-for the intake, water goes into the tube from every direction and water mainly focuses on the axis direction when it goes out. However, Jenkins ${ }^{68,69}$ uses a different way to explain the phenomenon from the point view of irreversibility. Even for the ideal flow described by the time-reversible Euler equation, just changing the sign of the pressure field to make the water intake and discharge would not reverse the flow exactly as shown in Equation (1).

$$
\left(\frac{\partial}{\partial t}+v \cdot \nabla\right) v=-\frac{\nabla P}{P}
$$

where $t$ is time, $v$ is velocity, $P$ is pressure, and $\nabla$ is the del operator. It is easy to see that for the same pressure field $P$, both $v$ and $-v$ could be the solution to this equation. However, changing the pressure $P$ into - $P$ would not make - $v$ the solution.

In other words, even for the ideal fluid, the intake and discharge flow patterns from the exhaust tube by changing the pressure gradient are still asymmetric. Therefore, the propulsion principle should be irreversibility instead of caused by boundary layer separation as stated by Dijkink et al. ${ }^{17}$. Because the phenomenon still exists in ideal fluid which does not have vicsocity, no boundary layer formed. For a more general case where the fluid has its viscosity, the explanation will be given in section 2.3.1. 


\subsection{ACOUSTIC BUBBLE MILLIMERTER SIZE}

Based on this idea, Dijkink et al. ${ }^{17}$ built the "acoustic scallop” which is a millimeter scale acoustic “putt-putt boat”, using an air bubble (200 $300 \mu \mathrm{m}$ diameter and $2 \sim 4 \mathrm{~mm}$ long) trapped in a one-end open Teflon tube. By applying an acoustic field at the resonance frequency of the trapped bubble $(1.55 \mathrm{kHz})$, which was generated by a piezoelectric transducer, the scallop was propelled by the bubble at a speed of about $1.35 \mathrm{~mm} / \mathrm{s}$. The theoretical calculation of the resonance frequency for the bubble in the tube was based on the spring-mass model developed by Chen et $a l .{ }^{70}$ and the previous experiments ${ }^{71}$. The propulsion force was calculated based on the observation that the fluid patterns for the bubble-shrinking and expansion processes were asymmetric, as shown in Figure 2.1 (a) and (b).

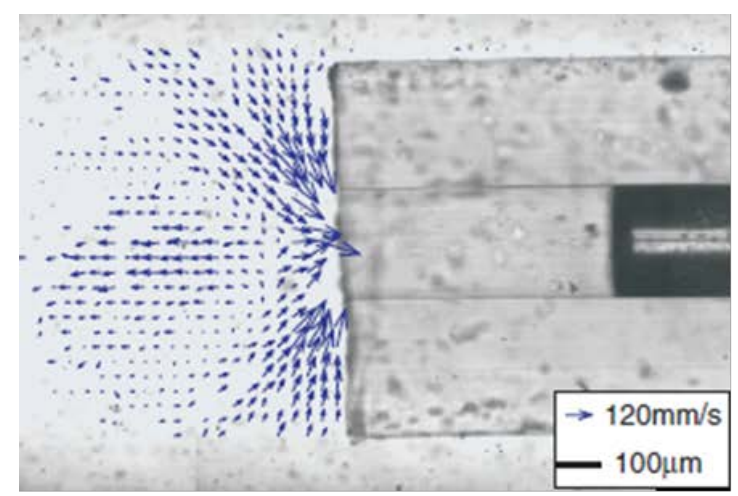

(a)

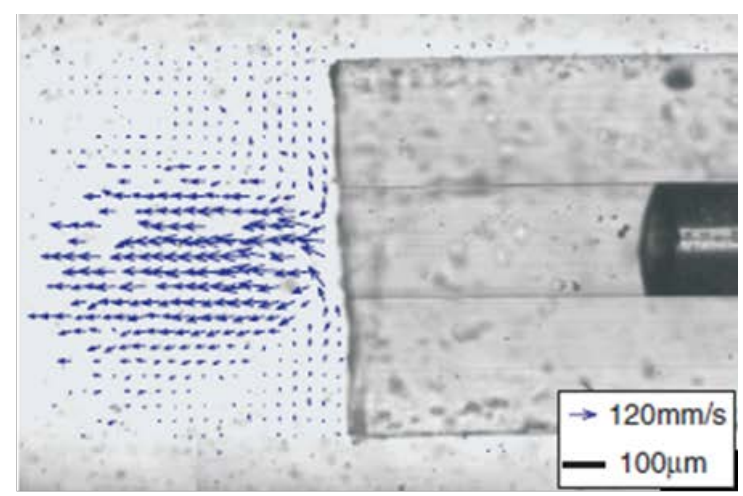

(b)

Figure 2.1. The flow field obtained from particle tracking velocimetry (PTV) at two phases of the acoustic cycle (a) phase $144^{\circ}$ (intake flow); (b) phase $216^{\circ}$ (outtake flow) [19]. 
The proper propulsion mechanism which is called "Machian Propulsion" is given by Jenkins $^{68,69}$ as stated before, caused by "irreversibility". If $R e=U L / v$ is defined using the speed and size of the body, $R e \sim 5$. One may ask this question: Since the propulsion is inertia dominant as stated before, why does propulsion mechanism still work at such as a low Re? The answer should be that if $R e$ is defined using the oscillation amplitude and speed of the bubble meniscus (the definition will be shown in later section 2.3.1), $R e \sim 63$, as the latter $R e$ of the generated flow is proportional to the operating frequency, it is high enough for the inertia to come into play to generate a net momentum transfer between the surrounding water and the "scallop" by oscillation of the bubble meniscus.

\subsection{ONE-DIMENSIONAL MICRON SCALE ACOUSTIC BUBBLE PROPULSION}

Although the concept of acoustic scallop is impressive and interesting, two major issues currently need to be addressed in order to bring it to in vivo biological or medical applications: (1) the dimensions of the device should be smaller such that it can possibly navigate through the space of arterioles and venules dimensions ( $>10 \mu \mathrm{m}$ in diameter). The critical dimension is the diameter of the bubble (or cylinder) rather than the length to pass through the narrow and serpentine passages. Here, it may be whether the acoustic bubble propulsion still work for smaller scales, and (2) it should have a steering capability to navigate and reach target sites.

This proposal attempts to mainly address these two issues. Regarding the first issue, we use the micro fabrication technology, as opposed to the conventional macro fabrication used in Dijkink et al. ${ }^{17}$, to make the bubble diameter in tens of micrometers. The backbone of the device is made of two parylene layers by micro photolithography (inset in Figure 2.2) such that the 
device is flexible. The maximum velocity of this device could reach over $40 \mathrm{~mm} / \mathrm{s}$ ( 80 body lengths/second), as presented in the later sections 2.3.3 with more details. At the same time, the basis for two-dimensionally steered motions is approved: by tuning the frequency of the external acoustic field, the resonance-matched bubbles can be selectively activated generating a strong propulsion force. In the future, the propulsion could be achieved by orthogonally arranging bubbles of different length as shown in Figure 2.2.

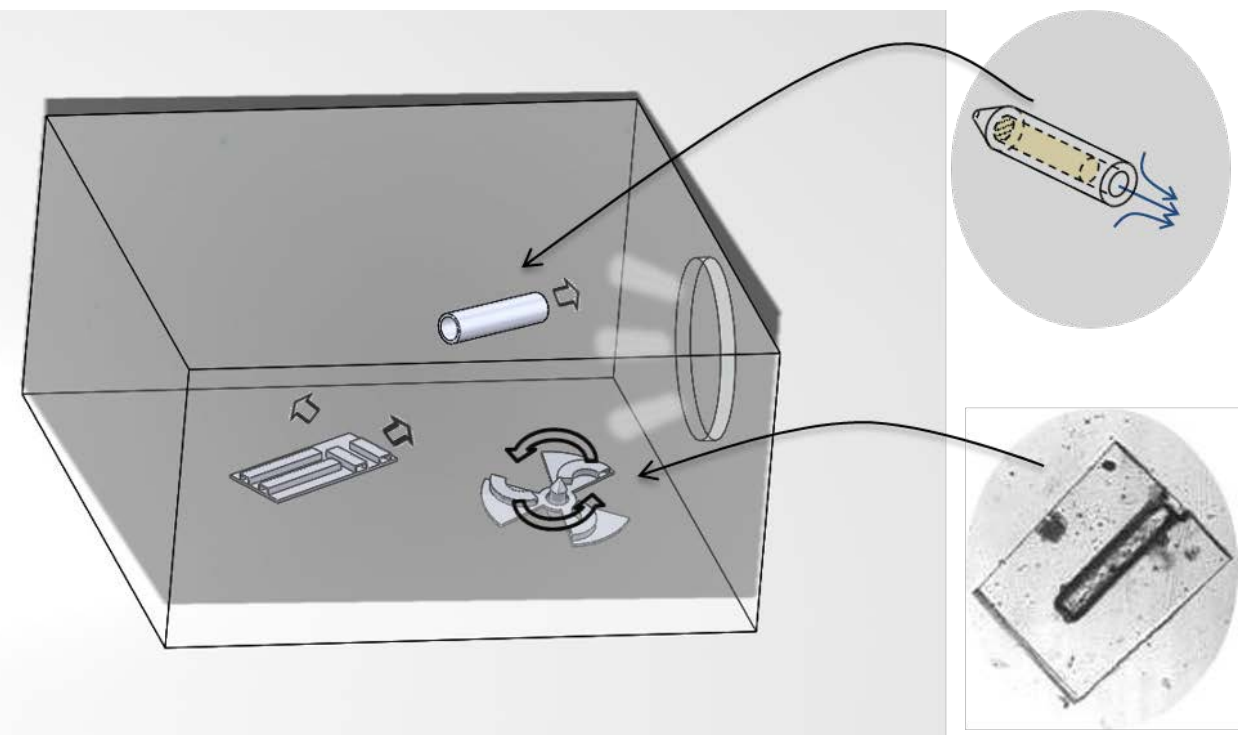

Figure 2.2. Concept of propulsion by oscillating bubble columns. Note that orthogonal arrangement of the bubbles can generate steering motion. The inset shows the fabricated single bubble device.

\subsubsection{Working Principle and Computational Fluid Dynamics Analysis}

The working principle of the present device is based on the acoustic scallop. A gaseous bubble is trapped in a microchannel with one end section open. When an acoustic field (pressure fluctuation) is applied to the bubble, the bubble meniscus at the open end of the microchannel 
periodically oscillates back and forth since the gas bubble is compressible and the other end of the microchannel is closed. This oscillation creates a periodical intake and discharge flow near the opening. Note that if $R e$ is large enough, flow fields of intake and discharge are not simply symmetric, the mean velocity field that is time-averaged over the period is not zero, which is called microstreaming flow. As a result, the magnitudes of total momentum flux and stresses on the device surface during the intake process would not be equal to those during the discharge process. On the other hand, the total mass flux through the channel during the intake process must be as during the discharge process unless there is mass accumulation or loss inside of the channel. Consequently, the difference in the momentum flux and stresses generates a propelling force acting on the device.

The reason for this asymmetric flow could be explained more generally as follows: for the common case, including both viscid and inviscid flow, the origin of this asymmetric flow comes from the nonlinear (inertia) term in the Navier-Stokes equations,

$$
\rho\left(\frac{\partial \vec{V}}{\partial t}+\vec{V} \cdot \nabla \vec{V}\right)=-\nabla p+\nabla \cdot T+f
$$

which is $\rho \vec{V} \cdot \nabla \vec{V}$ where $\vec{V}$ is the flow velocity, $p$ is the pressure, $\rho$ is the fluid density, $T$ is the component of the total stress tensor, $f$ represents the body force acting on the fluid and $\nabla$ is the del operator. This can be easily explained by replacing $\vec{V}$ with $-\vec{V}$ in the Navier-Stokes equation. Due to the nonlinear term, the replaced Navier-Stokes equation is not identical to the original Navier-Stokes equation. This means that supposing $\vec{V}$ is a solution of the Navier-Stokes equation, $-\vec{V}$ is not necessarily a solution to the Navier-Stokes equation. In other words, the flow generated by intake and discharge cannot be simply reversed but would be a different flow pattern. That is, asymmetric flow patterns should occur, which leads to the propulsion force. 
However, as the propulsion device becomes smaller, so does the $R e$ and thus the effect of the nonlinear term. In this case, the flow patterns during the intake and discharge processes become more symmetric, and the net momentum flux and thus the propulsion force become weaker. Eventually, when $R e=0$, the flow would perfectly symmetric ${ }^{72}$ and the propulsion force would be zero, since the effect of the nonlinear term has vanished. Therefore, $R e$ is a critical parameter to determine whether or not this propulsion works efficiently.

Here, the question is how small $R e$ can be reduced while still generating a substantial propulsion force. During oscillation, the velocity is time-varying. Using the peak velocity

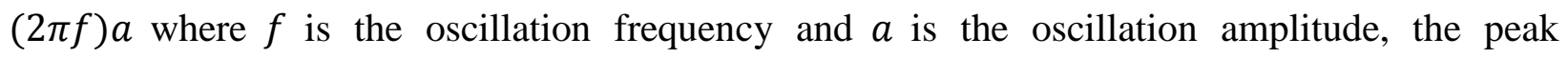
Reynolds number can be re-defined for the oscillatory flow as

$$
R e=\frac{2 R(2 \pi f) a}{v}
$$

where $R$ is the radius of the microchannel. Although the device becomes small (i.e., $R$ becomes small), it is possible to maintain the $R e$ at a certain range - where propulsion is still effective - by increasing the frequency $f$ and/or the amplitude $a$.

To investigate the effect of $R e$ on propulsion, two-dimensional computational fluid dynamics (CFD) simulations were carried out for simplified flow conditions using the commercial CFD package of ANSYS Fluent ${ }^{\circledR}$. 


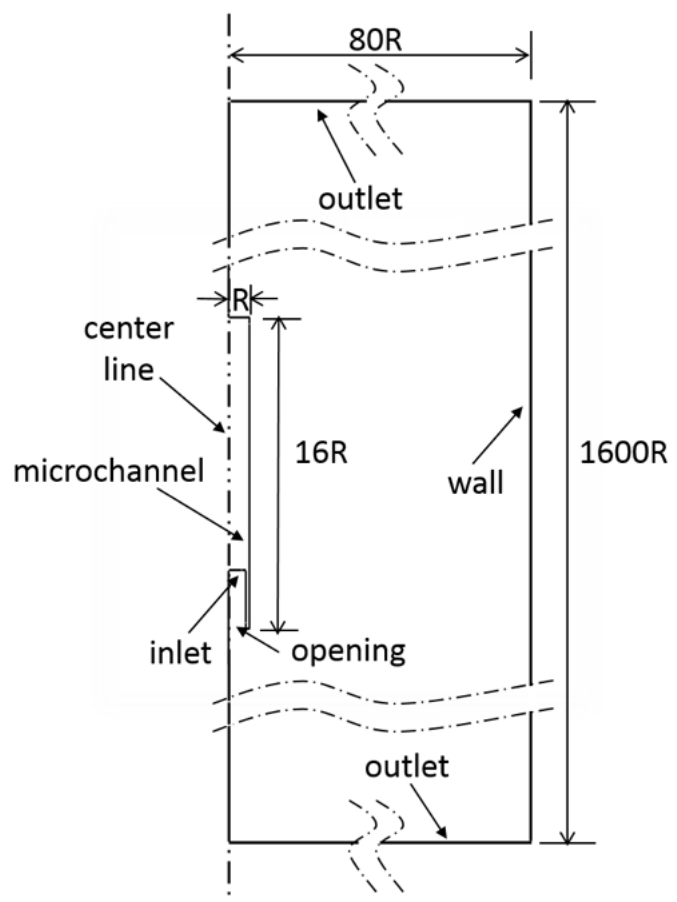

Figure 2.3. Computational domain of the bubble propulsion device placed and centered between two walls. Only the right half of the full domain is simulated under the assumption of flow symmetry between the left and right halves.

A one end open channel - which would hold a bubble in the device - is centered between two walls. Since the flow is assumed symmetrical between the left and right halves in the full physical domain, only the flow in the right half is simulated with a symmetric condition along the centerline, as shown in Figure 2.3. The width, length and wall thickness of the microchannel are $2 R, 16 R$ and $0.04 R$, respectively. The depth of the computational domain is set to be the unit length in ANSYS Fluent, which is $0.2 R$ in this case. The boundary conditions are that the upper and lower outlets of the domain are set to be 'outflow' and the no-slip condition is applied to the solid walls. For the sake of simplicity, the bubble is not included in the simulation. Instead, the uniform velocity profiles are assigned at $3 R$ on the inside from the edge of the opening 
(annotated as "inlet" in Figure 2.3). Although the oscillatory flow in the device is transient, two steady-state cases of (i) intake and (ii) discharge with a constant flow rate through the microchannel are computed and compared for various Re. This substantially simplifies the computation process yet allows us to understand the asymmetric behaviour between intake and discharge. The number of the computational grids is about 180,000.

Generally speaking, our simulation geometry is based on the same Re instead of the real size of our fabricated device which will be discussed in section 2.3.2. The reason is, first of all, the real dimension of our device will be in the scale of microns which is pretty small. What is more important, the real fluid velocity at the microbubble interface is very high, even up to the scale of meters per second. This is because of the very high bubble oscillation frequency, usually from several to ten kilohertz. Therefore, even the bubble oscillation amplitude is only tens of microns per second, the fluid velocity at the interface is still pretty high. This high speed compared with the small size of the device would bring a lot of difficulties to the simulation solution converge. Since the dominant equation, the Navier-Stokes Equation (2), is based on Re, there will be a balance between velocity and geometry size which could make the solution converge much easier. In other words, it could reduce the computation time a lot.

According to the idea of balance between velocity and geometry size, the velocity at the inlet is defined as follows. First of all, to roughly calculate the real fluid velocity at the inlet (microbubble interface) based on experimental data for the microbubble oscillation amplitude and frequency as section 2.3.3, the velocity is defined as,

$$
v_{\text {exp }}=2 f a
$$


where $v_{\text {exp }}$ is the experimental fluid velocity at the microbubble interface. Notice, this definition is different from the peak velocity ( $2 \pi f) a$ as defined in Equation (3). Then, to keep Re the same for simulation as discussed before,

$$
v_{\text {sim }} R_{\text {sim }}=v_{\text {exp }} R_{\text {exp }}
$$

and

$$
R_{\text {sim }}=100 R_{\text {exp }}
$$

as mentioned before, where $v_{\text {sim }}$ and $R_{\text {sim }}\left(R_{\text {sim }}=R\right)$ are the velocity at the inlet and half width of the microchannel for simulation. $R_{\exp }$ is the half width of the fabricated microchannel in the experiment. Because the real cross section of the fabricated microchannel is the trapezoid as mentioned in section 2.3.2.1, we take the average of upper and lower edge (80 $\mu \mathrm{m})$ as the $R_{\text {exp }}$ to use here. 


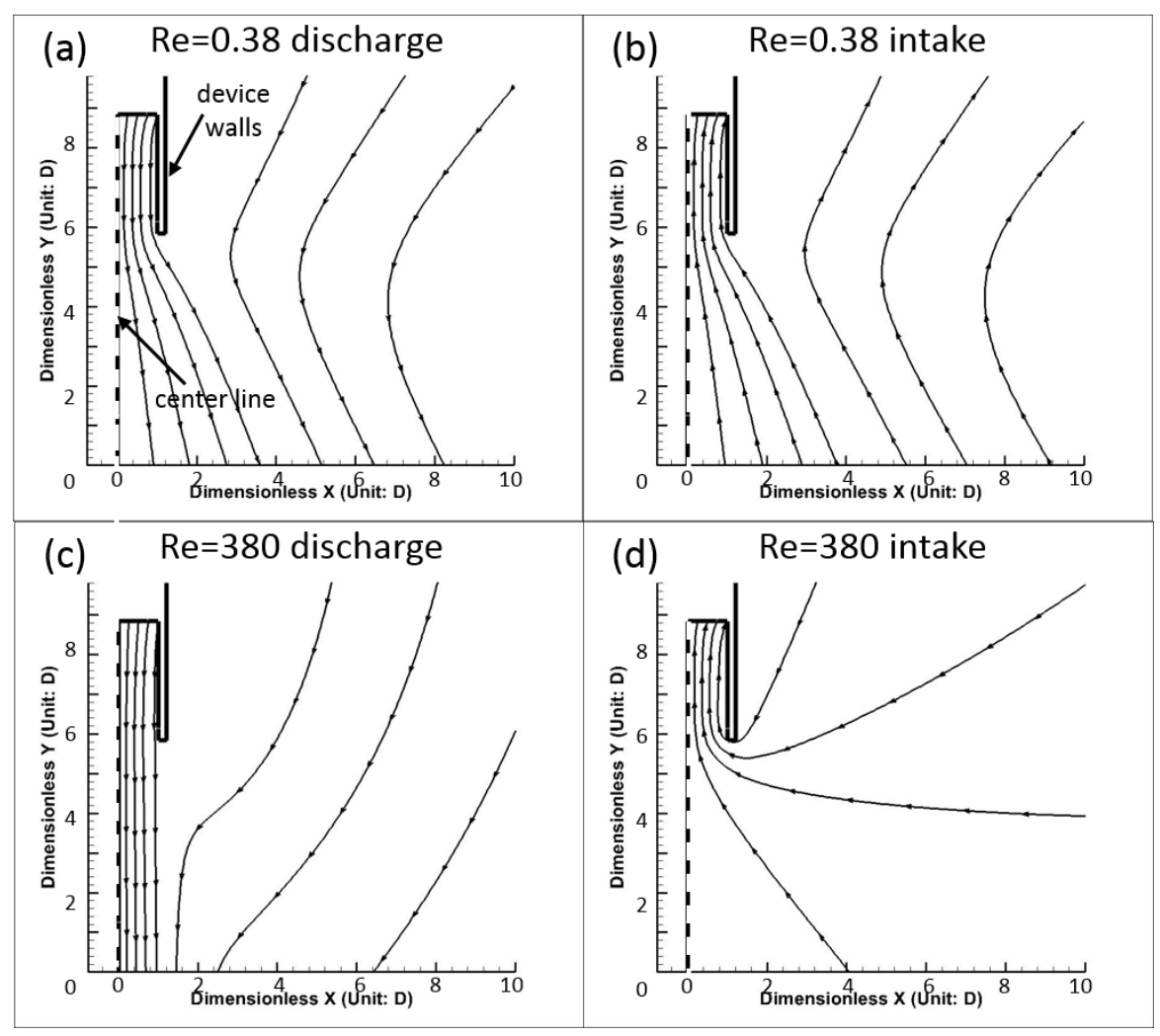

Figure 2.4. Numerical Results of streamlines for both discharge and intake cases at different Reynolds number (a) $R e=0.38$, discharge, (b) $R e=0.38$, intake, (c) $R e=380$, discharge and (d) $R e=380$, intake.

Five different $\operatorname{Re} 380,38,3.8,0.38$ and 0.038 are simulated for both intake and discharge cases to compare the effect of $R e$ to stream patterns. Re is changed by defining different fluid (water) velocities at the inlet of microchannels. Notice 380 is close to our experimental $R e$ in the following section 2.3.3, and the fabricated microswimmer successfully showed the underwater propulsion which means the asymmetric fluid patterns actually exist in this case. Therefore, 380 is used as the upper limit for $R e$. The lower limit is set as 0.038 to make sure it is small enough to show the symmetric flow pattern. Figure 2.4 shows streamlines, including in the region of outside the microchannels, for $R e=0.38$ and $R e=380$. The streamlines are re-drawn to make 
the flow rates for intake and discharge cases equal. The stream function value has an equal increment between the adjacent streamlines. As shown in Figure 2.4 (a) and (b) for $R e=0.38$, the streamlines for both intake and discharge are spread in a wide angle over the computational domain. Their distributions and patterns are almost the same for both cases. That is, the flow is symmetric between intake and discharge. The only difference is that the flow directions are opposite. That is, the flow is symmetric between intake and discharge. The magnitude difference in momentum and all the forces acting on the channel between the two cases would be nearly zero. That is, the net (propulsion) force would be almost zero if these two flows are alternated. On the contrary, when $R e=380$, the difference between intake and discharge is clearly distinct as shown in Figure 2.4 (c) and (d). For discharge, the streamlines starting from the opening of the microchannel extend straight in the axial direction of the channel. For intake, however, the streamlines that are almost evenly distributed in all direction are converged into the channel. The flow is even drawn from the backside of the channel opening. As a result, the propulsion force of the device should become substantial if the discharge and intake flows are periodically alternated.

The force acting on the channel (propulsion force) is calculated based on these twodimensional numerical results at several $R e$ numbers $(R e=0.38,1.9,3.8,19,38,190,380)$. A control volume for this calculation is defined identical to the outline (solid line) of the microchannel and its opening in Figure 2.3. The pressure, viscous stresses and momentum flux are integrated and calculated over the entire control surfaces. Then the sum of these integrations of intake and discharge cases is taken as the net force when the intake and discharge cases are alternated (no transient case considered). Notice that because the channel has no motion at all in our simulation, this net force actually means the external force applied to anchor this channel, 
which is always negative according to the simulation results. In other words, the negative (direction from the closed end of the channel pointing to the open end) force is applied to hold the position of the channel, and if the force is removed, the channel will get a propulsion force with the same magnitude of this holding force but in the opposite direction (direction from the open end pointing to the closed end). Therefore, the propulsion force could be calculated.
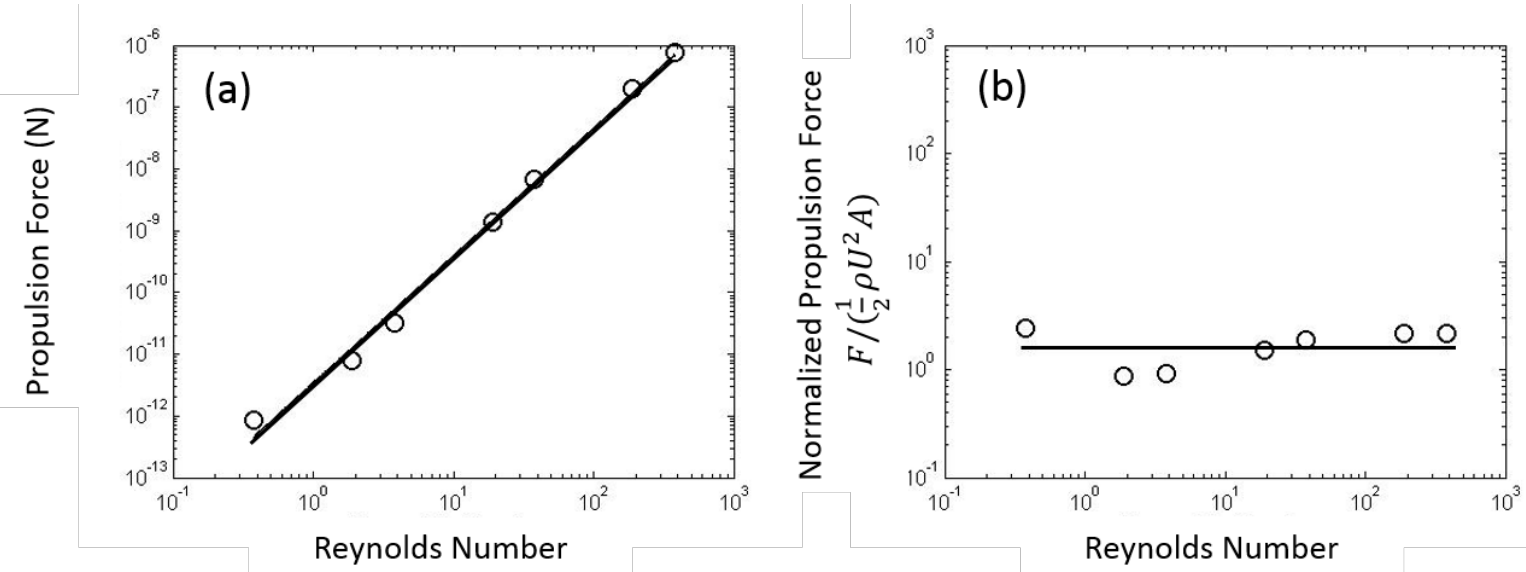

Figure 2.5. Numerical Results of (a) propulsion forces and (b) non-dimensionalized propulsion force at different Reynolds number.

Figure 2.5 (a) shows that the propulsion force monotonically increases as $R e$ increases. This is consistent with the streamline patterns in Figure 2.4 that the flow at the larger $R e$ becomes more asymmetric between intake and discharge. Figure 2.5 (b) shows the same data but the propulsion force is normalized by the momentum flux through the channel opening ( $\left.=\rho U^{2} A / 2\right)$ through the channel inlet, where $A$ is the cross-sectional area of the channel opening and $F$ is the propulsion force. Over the entire range of the simulated $R e$, the normalized propulsion force stays nearly constant. The propulsion force could be curve-fitted from Figure 2.5 (b), which 
gives the idea for the device design in the future, as Equation (7). Here, the standard deviation for the coefficient 0.8 is 0.29 . This simplest curve-fit makes it much easier to directly see the effects of the associated parameters, although it slightly compromises the accuracy compared to higher-order curve-fittings.

$$
F \cong 0.8 \rho A U^{2}=0.8 \rho A(a f)^{2}
$$

Equation (7) reveals that the propulsion force is proportional to the momentum flux through the channel opening: the oscillating velocity at the channel opening is a key factor to generate strong propulsion in given channel dimensions. Note that the magnitude of the oscillating velocity is the product of oscillating frequency and amplitude. That is, maintaining the high oscillating frequency and maximizing the oscillating amplitude is crucial to generate substantially strong propulsion forces.

Figure 2.6 shows all the simulation results for different $R e$ from 0.038 to 380, and the color of the background shows the velocity magnitude distribution. It is quite clear that the discharge flow becomes more and more focused on the axis direction as the $R e$ increases, and the magnitude becomes larger as a result. For example, when $R e=380$, the jet-like flow has a much higher impact to the downstream region compared with other Re. While for the intake flow, streamlines become more and more spread by increasing the Re. Consequently, the asymmetric flow pattern caused propulsion force should be larger for higher $R e$, which confirms the previous force calculation results. 

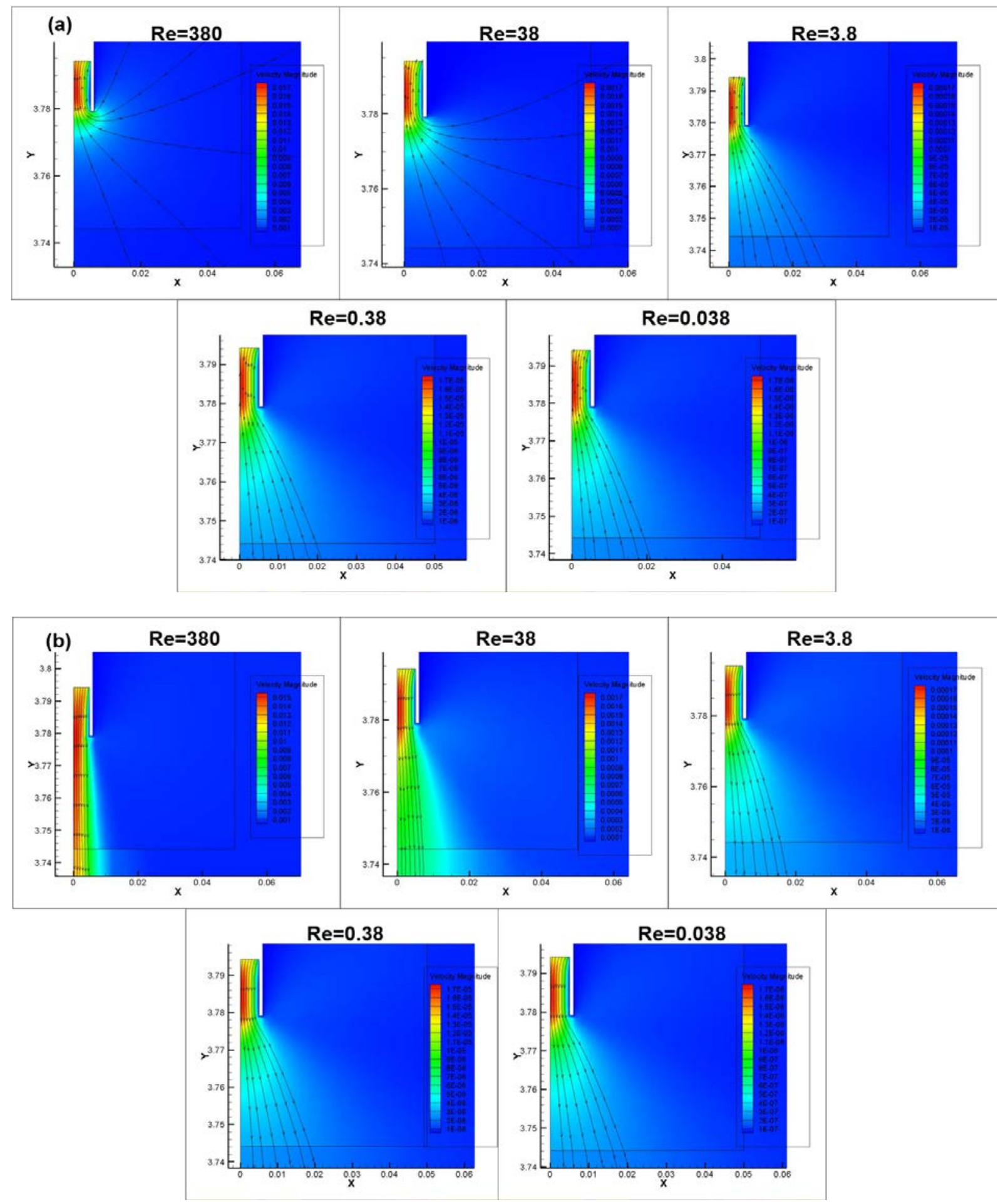

Figure 2.6. Numerical Results of streamlines and velocity magnitude for both (a) intake and (b) discharge cases at five different Reynolds numbers $R \boldsymbol{e}=380, \boldsymbol{R}=38, \boldsymbol{e}=\mathbf{3 . 8}, \boldsymbol{R} \boldsymbol{e}=\mathbf{0 . 3 8}$ and $\boldsymbol{R} \boldsymbol{e}=\mathbf{0 . 0 3 8}$. 
From the above simulation results, it can be seen that for given device dimensions, the $R e$ or the fluid velocity at the inlet is a key factor in generating a stronger propulsion force. What is more, oscillating amplitude is maximized when the bubble oscillation is resonated. Dijkink et al. suggested an expression on the resonant frequency $\left(f_{0}\right)$ based on the one dimensional springmass model theory ${ }^{73}$ :

$$
f_{0}=\frac{1}{2 \pi} \sqrt{\frac{\kappa P_{0}}{\rho L_{0} L_{B}}}
$$

where $P_{0}$ is the pressure of the undisturbed bubble, $\rho$ is the water density, $L_{0}$ is the length of water column between the bubble interface and the channel opening, $L_{B}$ is the length of the bubble, and $1 \leq \kappa \leq \gamma$ where $\gamma$ is the ratio of specific heats of gas in the bubble. Here, $\kappa$ is determined to be 1.2 for the present case based on Chen and Prosperetti ${ }^{70}$. Note that Equation (8) has no correction for the end effect ${ }^{74}$. Their determination and design of resonance condition was based on the previous experiments and numerical models ${ }^{70,71}$. They showed that Equation (8) was in good agreement with their experiments. In the present study, however, it turns out that predicting the resonance frequency is not easy, as it is highly influenced by experimental set-up including the materials, dimensions, and configuration of the testing water tank which will be discussed later in section 2.4.2. Therefore, we decided to directly measure the acoustic (oscillating) behaviour of the entire system set-up and bubble. These results are extremely beneficial in determining the length of the bubbles to maximize the bubble oscillation and will be used to arrange bubbles for two-dimensional propulsion. It is basic for two-dimensional propulsion control that orthogonally or oppositely aligned bubbles whose lengths are different are resonated at clearly distinct frequencies. 


\subsubsection{Device Fabrication and Experimental Set-up}

\subsubsection{MICROFABRICATION}

Testing devices were microfabricated based on micro photolithography. In selecting device material, the following requirements need to be taken into account. First, the microchannel surface has to be hydrophobic in order to hold a bubble inside the microchannel. The bubble can be automatically and easily trapped by simply submerging the microchannel in water. The bubble dimensions are defined by the microchannel dimensions even though a small water column will be formed at the open end of microchannel because of water coming in, otherwise, it is difficult to make a bubble in the exact size and shape. Second, the material should be transparent such that the bubble motion is observable during oscillation. In addition, it allows us to visualize the flow around the bubble meniscus by tracking seeded micro particles. Third, the density of the material should be neither too high nor too low that the overall density including the bubble is close to the water density. This ensures that the device will not float on the water surface and that the friction force with water tank surface would be minimized. Fourth, the material has to be biocompatible ${ }^{75}$ which means that the device made by the material has the ability to perform its function with an appropriate host response in a specific situation. Last, it is preferable that the material is flexible. This will facilitate navigation of serpentine passages. Parylene meets the above requirements. In addition, since parylene is deposited by the chemical vapor deposition (CVD) process, the deposition is highly conformal with accurate thickness control. 
(a)
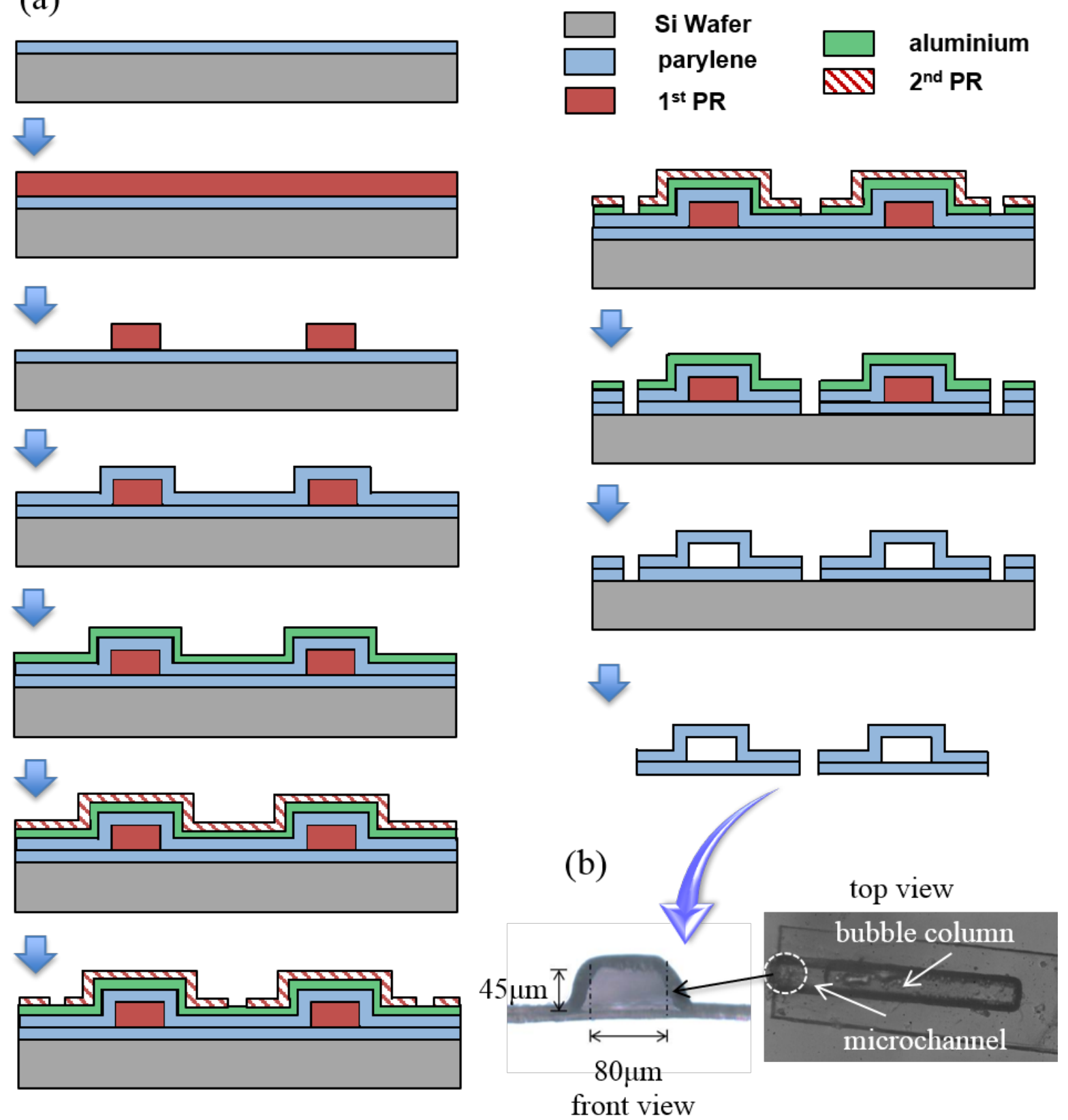

Figure 2.7. (a) Fabrication of one-end open microchannel and (b) photo of fabricated device (top view and frontal view at the channel opening). The microchannel traps and holds an air bubble column. One end of the channel is opened by RIE etching. 
The detailed microfabrication flow is shown in Figure 2.7, and Figure 2.8 shows the result for each step. It begins with the parylene coating process. The first $7 \mu \mathrm{m}$ thick parylene layer (C-type parylene, Special Coating System, INC.) is coated on a polished silicon wafer (4 inch wafer, University Wafer) through the CVD process (LABCOATER ${ }^{\circledR} 2$ Parylene Deposition Unit, Model PDS 2010, Special Coating System, INC.), which serves as the bottom wall of the microchannel. Notice that there is no sacrificial layer between the bottom parylene layer and silicon wafer. The reason is that the bonding between parylene and silicon is neither too weak nor too strong: the bonding is strong enough to hold the structures built on this bottom parylene layer to go through the whole fabrication process, including spin coating, photoresist development, dry etching and so on. There is no air gas or liquid droplet formed between these two layers when other fabrication processes are carried out, which makes the fabrication process more precise. At the same time, the parylene and silicon could be separated automatically by submerging them in acetone for several minutes, which makes it so the sample can be peeled off from the substrate in the final fabrication process, which is even easier as compared with a thin photoresist layer as a sacrificial layer. If the thickness of the parylene layer is too thick, it will make the device become heavy enough to sink, contact the bottom wall of testing chamber, and increase friction with the wall. On the other hand, if the thickness is too low, the strength of the microchannel is reduced, which allows the channel itself to expand and shrink when the bubble is oscillated by the applied acoustic field. This would eventually reduce the oscillation amplitude of the bubble meniscus at the open end of microchannels and thus propulsion force. It turns out that the $7 \mu \mathrm{m}$ thickness is suitable for not confronting the above issues.

The next step is to deposit a $45 \mu \mathrm{m}$ photoresist $\left(\mathrm{AZ}{ }^{\circledR} \mathrm{P} 4620\right.$ Photoresist, AZ Electronic Materials) layer on the parylene layer. To get this thickness, a double layer coating process 
(AZ ${ }^{\circledR}$ P 4620 Photoresist Data Package, AZ Electronic Materials) is used with a thickness of about $24 \mu \mathrm{m}$ for each by the spin coater (Model CT/62, Karl Suss). The lower $24 \mu \mathrm{m}$ photoresist layer is coated with a spin speed of $800 \mathrm{rpm}$, an acceleration of $500 \mathrm{rpm}$, and total spin time $45 \mathrm{~s}$. After spin coating, $7 \mathrm{~min}$ soft baking is finished on a hot plate for the first photoresist layer at $105^{\circ} \mathrm{C}$ to evaporate the solvent in photoresist and make it solid. After the soft baking, the sample is cooled to room temperature and the upper $24 \mu \mathrm{m}$ photoresist layer is built, using exactly the same method as the lower one. Thus, a total $48 \mu \mathrm{m}$ photoresist layer is derived.

The $48 \mu \mathrm{m}$ photoresist layer is patterned by photolithography using a mask aligner (Model MA6, Karl Suss) as a sacrificial layer then developed (AZ ${ }^{\circledR} \mathrm{P} 400 \mathrm{~K}$ Developer Diluted 1:3, AZ Electronic Materials) to define the microchannel. The exposed photoresist layer will be gone during development process and only bottom parylene layer will be left. It provides the space to adhere the first parylene layer, as the bottom wall of the microchannel, with the second parylene layer which is deposited later as the top and side walls of the microchannel. The remaining photoresist layer part which will be covered by the second parylene layer later, helps the second parylene layer to form the shape of the microchannel.

Then, the second $7 \mu \mathrm{m}$ parylene layer is deposited on the remained photoresist layer to form the upper wall and side walls of the microchannels. These two parylene layers could be bonded during vapor deposition process automatically.

Next, an aluminum layer about $700 \mathrm{~nm}$ thickness (Purity 99.999\%, Angstrom Sciences, INC.) is coated by the sputtering process (Innovative, System Engineering, INC.) for 15 minutes which will work as the mask for the following reactive ion etching (RIE) process. This aluminum mask layer is patterned by wet etching with the second $2 \mu \mathrm{m}$ photoresist layer at a spin speed of 3000 rpm, $1000 \mathrm{rpm}$ acceleration and total spin time of $45 \mathrm{~s}\left(\mathrm{AZ}^{\circledR} \mathrm{P} 4000\right.$ Series Spin Speed Curve, AZ 
Electronic Materials, AZ ${ }^{\circledR} \mathrm{P} 4210$ Photoresist, AZ Electronic Materials). The aluminum layer can be simultaneously etched by an alkaline-based photoresist developer $\left(\mathrm{AZ}^{\circledR} \mathrm{P}\right.$ 400K Developer Diluted 1:4, AZ Electronic Materials) as the second photoresist layer is developed. This aluminium layer is utilized as protection when one end of the microchannel is open and the device is diced by the reactive ion etching (RIE) process (Phantom Ш LT, Trion Technology). The area which is not covered by aluminum could be etched away by $\mathrm{CF}_{4}$ and $\mathrm{O}_{2}$ plasma, including the second parylene layer, sacrificial photoresist layer, and first parylene layer. The etching rate for photoresist and parylene are not far from each other, and the total etching time is about 2 hours.

After the RIE process, all aluminum residue is removed by the alkaline-based photoresist developer. Finally, the sample with silicon substrate is submerged into an acetone solution (Semiconductor Grade, Ultra Pure Solutions, INC.) to remove the sacrificial photoresist layer inside the parylene microchannels. The removal time is about 2 hours to completely remove the photoresist residue. During this step, the microchannels could be separated from the silicon wafer automatically because the weak adhesion force between bottom parylene layer and silicon substrate. The front and top views of the fabricated microchannel are shown in Figure 2.7 (b). When dicing the device, a sufficient margin is deliberately given such that the channel has a flap around it. This is only for easily handling in device testing. Whenever needed, the flap can be easily removed by changing the mask design. All the microchannels fabricated have the same height of $45 \mu \mathrm{m}$ and width of $80 \mu \mathrm{m}$. However, the microchannels have different lengths, such as $270 \mu \mathrm{m}, 530 \mu \mathrm{m}$ and $820 \mu \mathrm{m}$, and different shapes, such as straight microchannels and spiral one, but all of them have similar cross sections. The equivalent diameter $(60 \mu \mathrm{m})$ of the present microchannel is over 12 times smaller than as in Dijkink et al. ${ }^{17}$. 


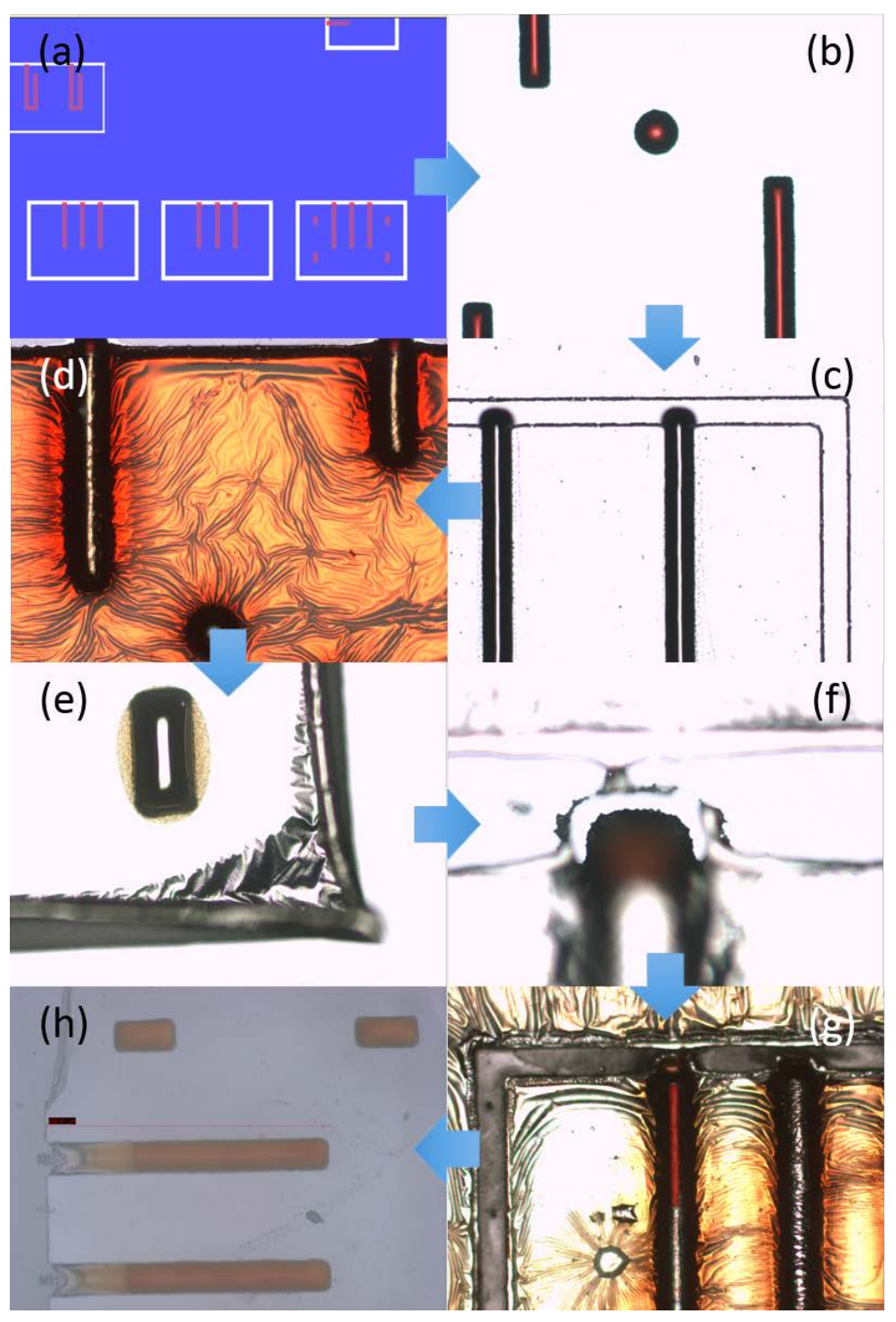

Figure 2.8. Fabrication steps (a) two masks design, (b) after first and second parylene coating and sacrificial photoresist pattern, (c) after Al mask sputtering, (d) after second photoresist pattern, (e) after RIE etching, (f) a close view of the open end of microchannel, (g) Al mask etched by acetone and (h) sacrificial photoresist etched by acetone. 


\subsubsection{EXPERIMENTAL SET-UP}

For acoustic actuation, a focused transducer may be used, whose acoustic output does not rely on the acoustic properties of the surrounding system. However, as the swimming device moves, the focused transducer may require a complex control system to trace the swimming device and to steer the acoustic output. To simplify the overall system setup, the acoustic field is generated by a non-focused disk-type piezoelectric actuator ( $27 \mathrm{~mm}$ in diameter and resonated at 4.6 +/-0.5 $\mathrm{kHz}$ ) glued to the side wall of the water tank. The input signal is generated by a function generator (20 MHz Function/Arbitrary Waveform Generator, Model 33220A, Keysight (Agilent) Technologies) and amplified by an amplifier (Piezo Driver/Amplifier Series Dual Channel, Model PZD700, TREK, INC.). The signal to the actuator is monitored by an oscilloscope (200 MHz 2.5 GS/s, Model 199C Scopemeter Color, Fluke Corporation of Sale). The testing water tank is $11 \times 11 \times 5 \mathrm{~cm}^{3}$ and made of transparent acrylic plates ( $3 \mathrm{~mm}$ thick). The experimental images are bottom view through the water tank and obtained by an inverted microscope (Eclipse TE2000-U, Nikon) connected to a high-speed camera (Phantom V7.3, Vision Research, INC.). Different frames rates, from 30 to 36,000 frames per second, are used for different purposes, such as bubble oscillation amplitude measurement, fluid pattern measurement with seeded particles and propulsion velocity measurement. A miniature reference hydrophone (TC 4013, Teledyne RESON) is used to measure the amplitude of acoustic field generated by the piezoelectric actuator. The hydrophone is held vertically and its tip is kept $1 \mathrm{~mm}$ away from the water tank bottom. 
The testing microswimmer device is always put on the bottom of the water tank. Since the average density is a little bit higher than that of water, it will not remain suspended in water. Another important reason to put it on the bottom is the acoustic wave transfer behavior of the system. The acoustic wave transfers from the acrylic testing water tank to water through bending waves as a water-loaded system. Based on the infinite water-loaded model, the pressure field in water could be expressed as the following equation

$$
P(x, y, t)=\frac{c \rho_{0} u_{y p \max }}{\left(1-k_{B}^{2} / k^{2}\right)} e^{i \omega t} e^{-i k_{B} x} e^{-i y\left(k^{2}-k_{B}^{2}\right)^{1 / 2}}
$$

where $P$ is the pressure, $x, y$ are the position coordinates, $t$ is time, $\omega$ is the angular frequency of the acoustic wave, $u_{\text {ypmax }}$ is the maximum oscillation velocity of the acoustic wave in $y$ direction, $k_{B}$ is the bending wave number inside the solid which is acrylic in our system and $k$ is the wave number in the fluid which is water. It is clear that if $k=k_{B}$, then $k^{2}-k_{B}^{2}=0$, therefore there is no acoustic pressure attenuation in $y$ direction. If $k>k_{B}$, then $k^{2}-k_{B}^{2}>0$ and $-i y\left(k^{2}-k_{B}^{2}\right)^{1 / 2}$ will be imaginary numbers, therefore there would be acoustic pressure field oscillation in $y$ direction. If $k<k_{B}$, then $k^{2}-k_{B}^{2}<0$ and $-i y\left(k^{2}-k_{B}^{2}\right)^{1 / 2}$ will be real number therefore acoustic pressure will be exponentially decay in $y$ direction which would result in a near field acoustic field ${ }^{76}$. In other words, the amplitude of the acoustic wave decreases very fast in the exponentially trend. In our case, for a frequency of around $10 \mathrm{kHz}, k<k_{B}$ according to the calculation. As a result, in order to have a relatively stronger oscillation amplitude of the bubble meniscus, which requires higher pressure field amplitude, the mircroswimmer device is always put on the bottom wall of the water tank. The acoustic measurement results will be shown in the next section 2.3.3. 


\subsubsection{Experimental Results}

For one-dimensional propulsion, a device with multiple $530 \mu m$ long microchannels is examined in the testing tank with a water level of $3 \mathrm{~cm}$. When the device is submerged in water, an air bubble is automatically trapped inside each microchannel. The bubble length is about 470 $\mu m$ and the width of the bubble is about $80 \mu m$. The remaining section (about $60 \mu m$ ) of the microchannel is filled with water. In this experiment configuration, to find the bubble oscillation amplitude, the frame rate of a high speed camera is set at 88,000 frames per second which can capture more than 6 images for each bubble oscillation cycle. The bubble oscillation amplitude is always measured by the difference between maximum convex and minimum concave bubble menisci. The acoustic field is applied sweeping the frequency from $1 \mathrm{kHz}$ to $14 \mathrm{kHz}$ with an equal increment of $0.1 \mathrm{kHz}$ to find the maximum oscillation amplitude of the bubble. Equation (8) predicts the resonance frequency for the present bubble configuration to be $10.5 \mathrm{kHz}$ while it is experimentally measured that the bubble oscillation is at its maximum at $11.2 \mathrm{kHz}$. The reason for discrepancy is discussed later in this section.

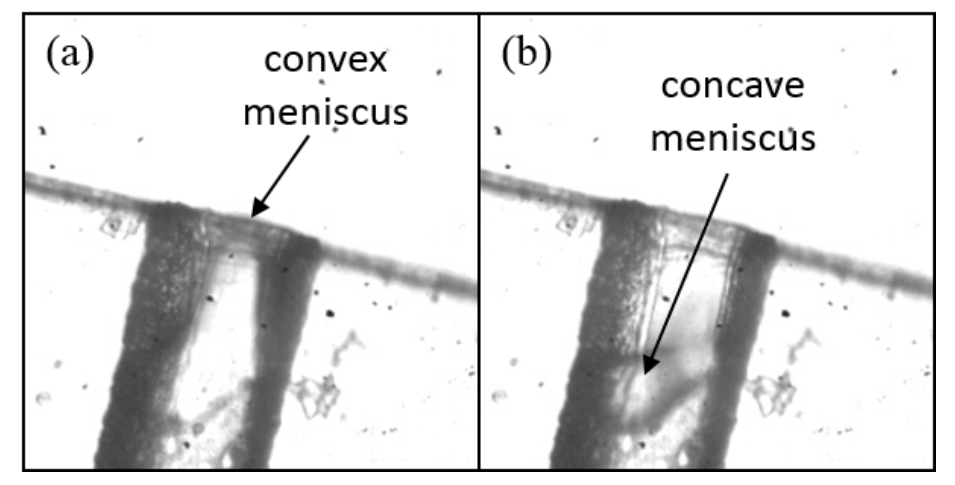

Figure 2.9. Bubble meniscus formed in one oscillation cycle (a) convex and (b) concave meniscus. 
The device with an array of ten microchannels is anchored to the bottom of the tank and the image is only focused on one of the microchannels. When the piezoelectric actuator is turned on, the bubble meniscus is oscillated back and forth periodically. When the bubble expands, the meniscus profile becomes convex out in the opening and the water in the channel is discharged as shown in Figure 2.9 (a). The discharge flow is pointed mainly in the axial direction of the channel. When the bubble shrinks, the meniscus is concaved into the microchannel as shown in Figure 2.9 (b). Consequently, water is drawn into the channel from all directions.

To see whether the streaming flow is generated near the opening of the channel, flow visualization is first performed by seeding $2 \pm 0.5 \mu \mathrm{m}$ diameter borosilicate glass micropspheres tracers (Duke Scientific Corporation) in water. Due to the motion of meniscus, the microparticles are also moving in the oscillating fluid. From high-speed camera images at the frame rate of 88,000 frames per second, it is found that oscillations of the microparticles and meniscus are exactly synchronized with the driving frequency, which confirms that the acoustic energy is the source for the fluid motion.

The microparticles along the microchannel axis have larger displacements in the expanding process to get away from the open end of the microchannel than in the shrinking process to approach the open end. The microparticles near the rim of the channel opening are the opposite: smaller displacement in the microbubble expanding process to get away from the open end but larger displacement in the shrinking process to get close to the open end. As a result, the microparticles along the axis are expelled from the opening and the particles far off the axis are attracted to the opening in one oscillation cycle. If this net displacement formed in one expanding and shrinking cycle is accumulated tens of thousands of times, it will make a jet shape microstreaming from the open end of the microchannel along the axis direction and two ring 
shaped vortices at the open end, symmetric to the jet (top view). To confirm this, the microstreaming is also visualized by the seeding microspheres with a high speed camera at a low frame rate of 200 frames per second. The image shows that the microspheres are entrained from the sides of the cylinder tip and strongly ejected from the cylinder tip as shown in Figure 2.10 (a) to (c).

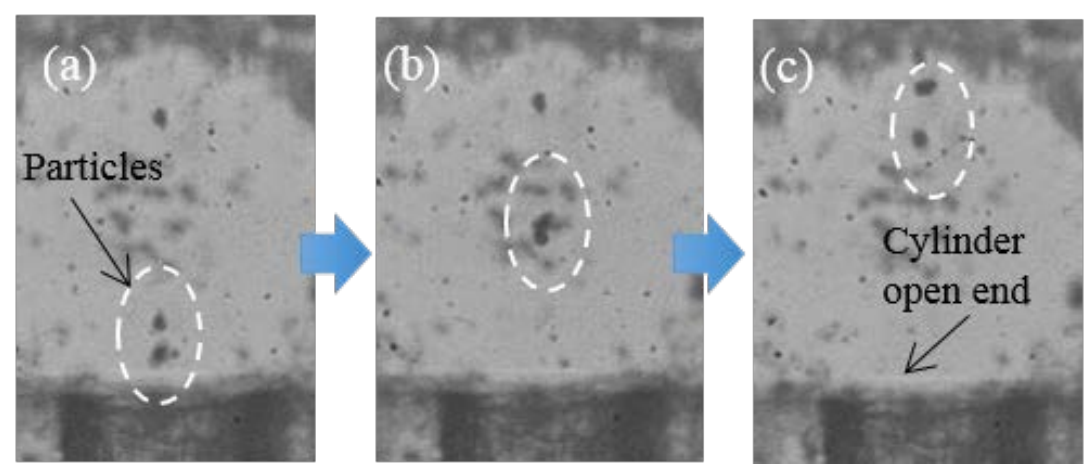

Figure 2.10. Flow visualization of streaming flow. The particles are pushed away from the cylinder openend when the bubble is oscillating.

As time goes by, the generated microstreaming sweeps the particles and creates a particleempty region as shown in Figure 2.11. The arrows are added to describe the straight outgoing flow along the axis and two vortices on the sides of the channel opening. The size of the empty region increases as the input voltage to the piezoelectric actuator increases (Figure 2.11from (a) to (c)). This is because increasing the oscillation amplitude will generate a stronger propulsion force $F$, as mentioned previously in Equation (7). To compare the sizes of different empty regions, each image of the Figure 2.11 is captured about $5 s$ after the piezoelectric actuator is turned on. 

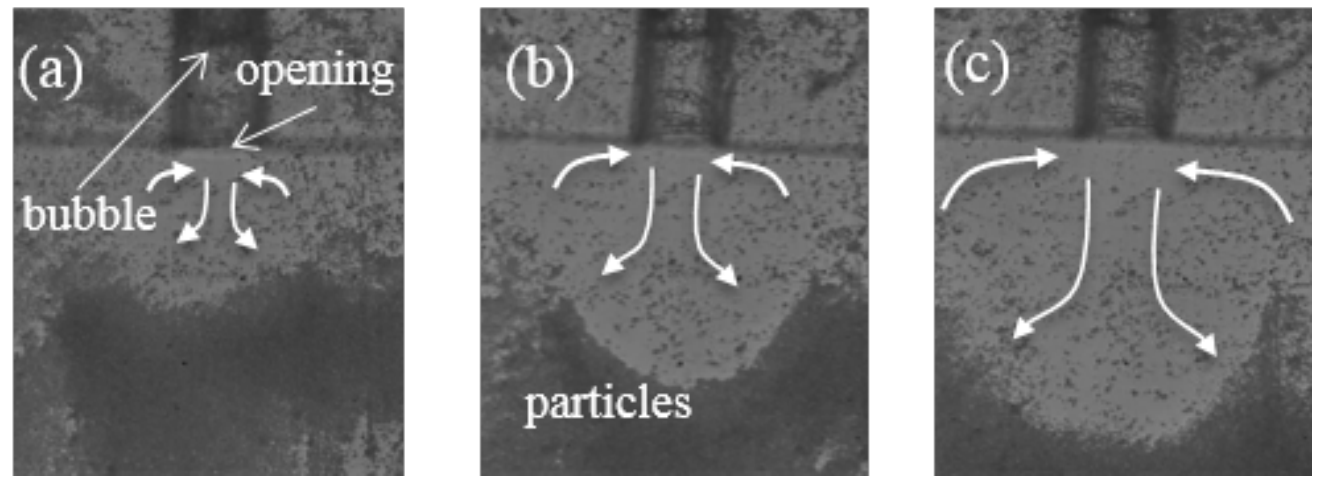

Figure 2.11. Generation of streaming flow. After one min. bubble oscillation, seeding particles are pushed away from the cylinder open-end, as the empty region is distinctively seen. Applied voltages to piezo-actuator are (a) 77 (b) 126 and (c) $165 V_{p p}$.

To see if this time-averaged flow is strong enough to propel the device, a single bubble device is placed on the tank bottom after removing the anchor. If the generated propelling force is larger than the resistance caused by fluid drag and friction from the tank bottom, the device should be able to be propelled. Figure 2.13 (a) shows snapshots of successful propulsion. The device is propelled to the bottom-left corner.

Here, a question is whether this propulsion is really generated by the streaming flow or by other mechanisms. Apparently, there are other possible mechanisms for this propulsion: for example, (1) global acoustic streaming which is generated between the interfaces of tank walls and water ${ }^{77}$; and (2) asymmetric friction between the device and tank walls during oscillation. In order to verify the truly responsible mechanism, the device is placed on a suspended glass plate in water that maintains a $2 \mathrm{~mm}$ gap above the tank's bottom wall. This plate is hung on an isolated beam and is not connected to the water tank directly therefore the oscillation from the water tank to this plate is isolated. Then, the direction of propulsion is checked for four initial 
orientations $\left(0^{\circ}, 90^{\circ}, 180^{\circ}\right.$, and $\left.270^{\circ}\right)$. For all the four orientations, the device is tested with the same input signal given (11.3 $\mathrm{kHz}$ and $220 V_{p p}$ ). Regardless of the initial orientation, the device is always propelled opposite to the opening side when the acoustic signal is applied as shown in Figure 2.12. This means the streaming flow through the opening generates a pushing force since neither the acoustic streaming nor the asymmetric friction is closely correlated to where the opening is. It is concluded that these two mechanisms are excluded from the responsible propulsion mechanism. The microstreaming caused by acoustic bubble oscillation is the only way to make it.

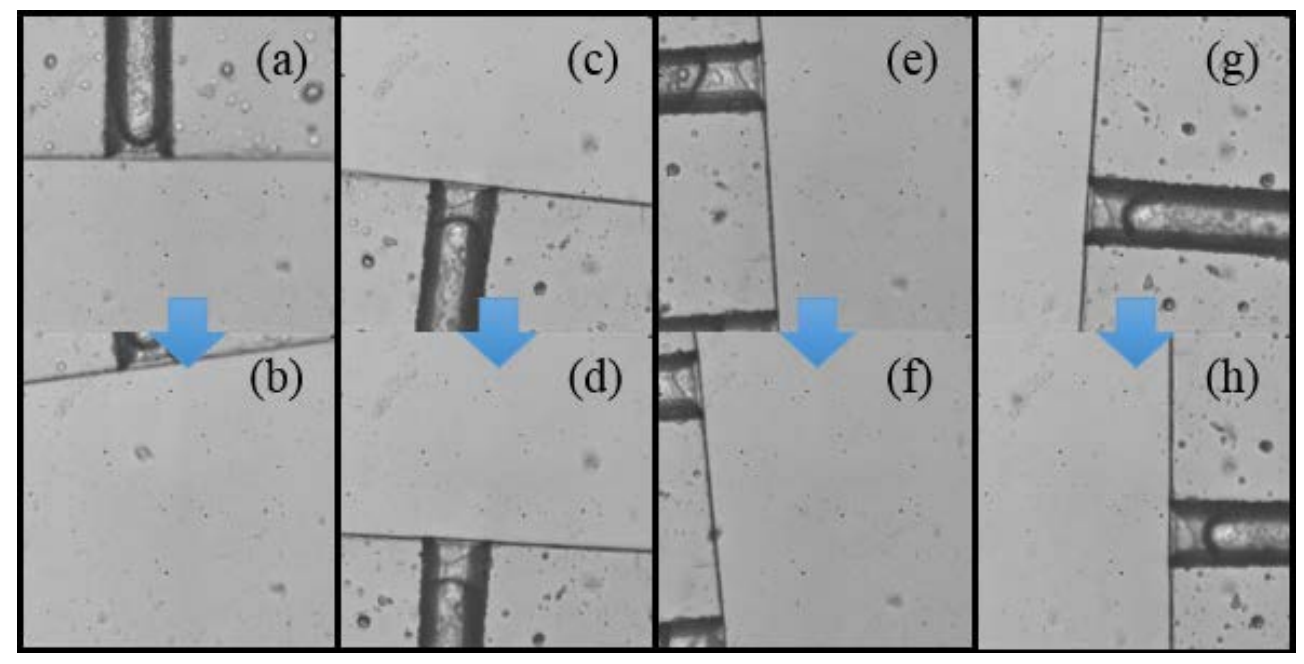

Figure 2.12. Propulsion verification: two consecutive snapshots for different orientations of the cylinder with respect to the testing tank; (a) and (b) moving up. (c) and (d) moving down. (e) and (f) moving left. (g) and (h) moving right.

The device $(5.4 \mathrm{~mm} \times 780 \mu \mathrm{m})$ with a channel array is also tested which has 10 microchannels parallel to each other and whose channel openings face the same direction, as shown in Figure 2.13 (b). The input voltage is always fixed at $170 V_{p p}$. When the driving frequency is also set at 
$11.2 \mathrm{kHz}$, the device shows the best performance in propulsion. Figure 2.13 (b) shows sequential snapshots of the motion at a speed of $3.87 \mathrm{~mm} / \mathrm{s}$. The propulsion direction is changed slightly due mainly to non-uniform friction between the device and the bottom of the testing tank. This arraytype device can be attached to any curved surface to propel large objects.

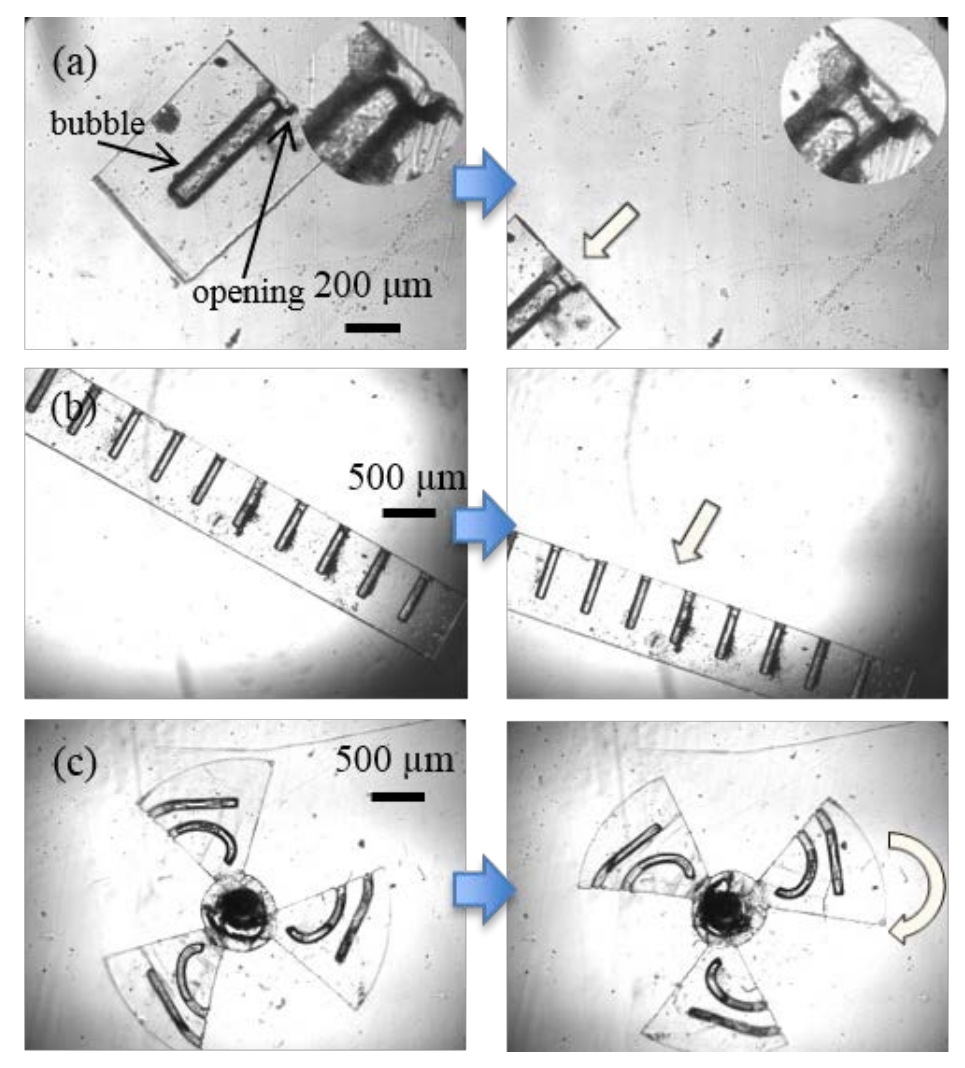

Figure 2.13. (a) Propulsion by a single bubble $\left(11.2 \mathrm{kHz}, 170 V_{p p}\right.$; see supplementary video clip 2, 60x slower; Insets show bubble menisci and channel opening), (b) Propulsion by an array of bubbles (11.2 kHz, $170 \mathrm{~V}$; see supplementary video clip 3, 16x slower), (c) Rotation by bubbles embedded on turbo blades (3.8 $\mathrm{kHz}, 480 V_{p p}$; see supplementary video clip 4, 1.1x faster) and (d) Payload carrying by an array of bubbles (11.2 kHz, $190 \mathrm{~V} \mathrm{pp}_{\text {; }}$ see supplementary video 5, 60x slower for translation and 25x slower for rotation). The inset shows a side view of the arrayed bubbles and load. Note that due to aliasing of image sampling the bubble oscillations look slower than the applied frequency. 
The rotation of the device could also be achieved by embedding bubbles on the turbo blades, as shown in Figure 2.13 (c). There are six $820 \mu \mathrm{m}$ microchannels on three turbo blades: two on each blade. The radius of the turbo device is $1750 \mu \mathrm{m}$ and the device is anchored at the center by an optical fiber at the bottom of the testing tank. The microchannels are curved to fit in the blades. The signal is reduced to be $3.8 \mathrm{kHz}\left(480 V_{p p}\right)$ due to the increased bubble length. The blades rotate continuously in a clockwise direction at $75 \mathrm{rpm}$. This rotational motion can be utilized and applied to drive micropumps, microvalves, micromixers, etc.

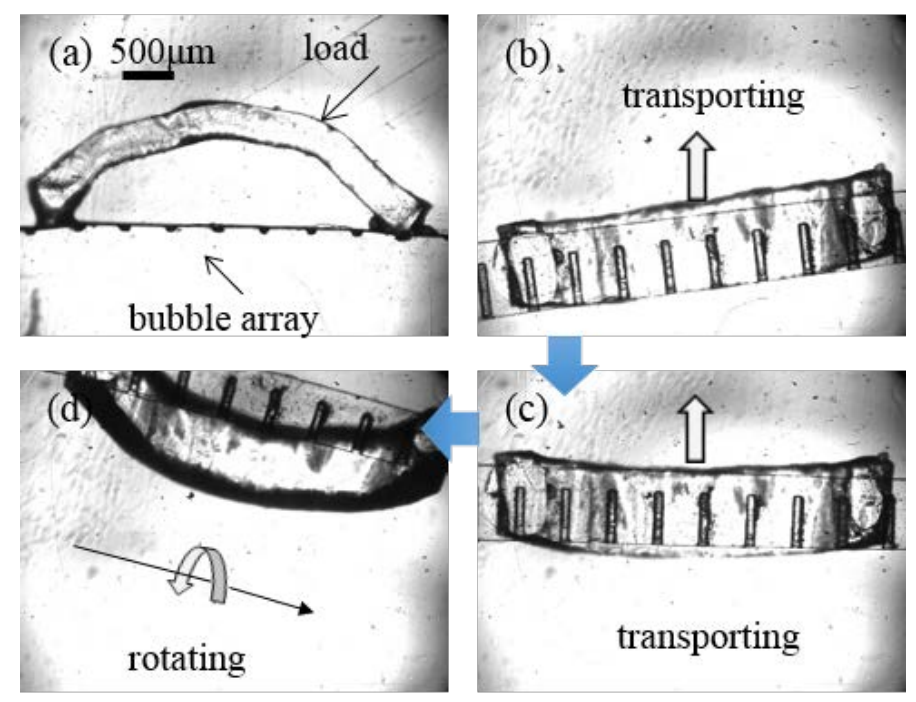

Figure 2.14. An array of oscillating bubbles carries a load of several millimeters in size: (a) initial configuration; and (b) transporting and rotating of the load by the bubble array.

Carrying a payload is also examined. A testing load is made of a thin plastic film that is bent and glued to the two ends of the arrayed device as shown in Figure 2.14 (a). Because of the increased mass and surface area, friction from the tank bottom and drag are increased. It is difficult to get a similar propulsion using the previous voltage as in Figure 2.13 (a) and (b) (170 
$V_{p p}$ ): A higher voltage of $190 V_{p p}$ is used. When the bubbles oscillate at $11.2 \mathrm{kHz}$, the device with the load is pushed away as in Figure 2.14 (b) and (c). This arrayed device is also able to rotate the load as in Figure 2.14 (d). Because the arrayed device is only attached to one side of the load, the propulsion force generates a torque that eventually rotates the load.

The effects of the input voltage and frequency on the propulsion speed are examined, as shown in Figure 2.15. At $11.2 \mathrm{kHz}$, the propulsion speed increases as the voltage increases (Figure 2.15 (a)). The maximum velocity is even over $45 \mathrm{~mm} / \mathrm{s}$, about 50 body lengths/second. If the flap were removed, the velocity would be higher due to reduced friction and drag. The propulsion force $F$ is estimated to be $\sim 0.8 \mu N$ by Equation (7). When the input voltage is higher than $200 V_{p p}$, the bubble oscillates so violently that the bubble trapped in the channel is broken into smaller bubbles. At this moment, the propulsion speed is even larger, because the extra small bubbles caused by the large bubble broken will also go out from the open end of this microchannel which will give an extra moment transfer compared with only bubble oscillation. This results in reduction of the bubble size in the channel.

In addition, it was observed that the bubble size was reduced after oscillation ( 20\% after 1hour oscillation). The main reasons for reduction are that the strong oscillation enhances the fluid mixing and thus gas dissolution and occasionally segregates the mother bubble into much smaller daughter bubbles. As a result, the resonance frequency was observed to shift and thus the life time of the device is reduced. 

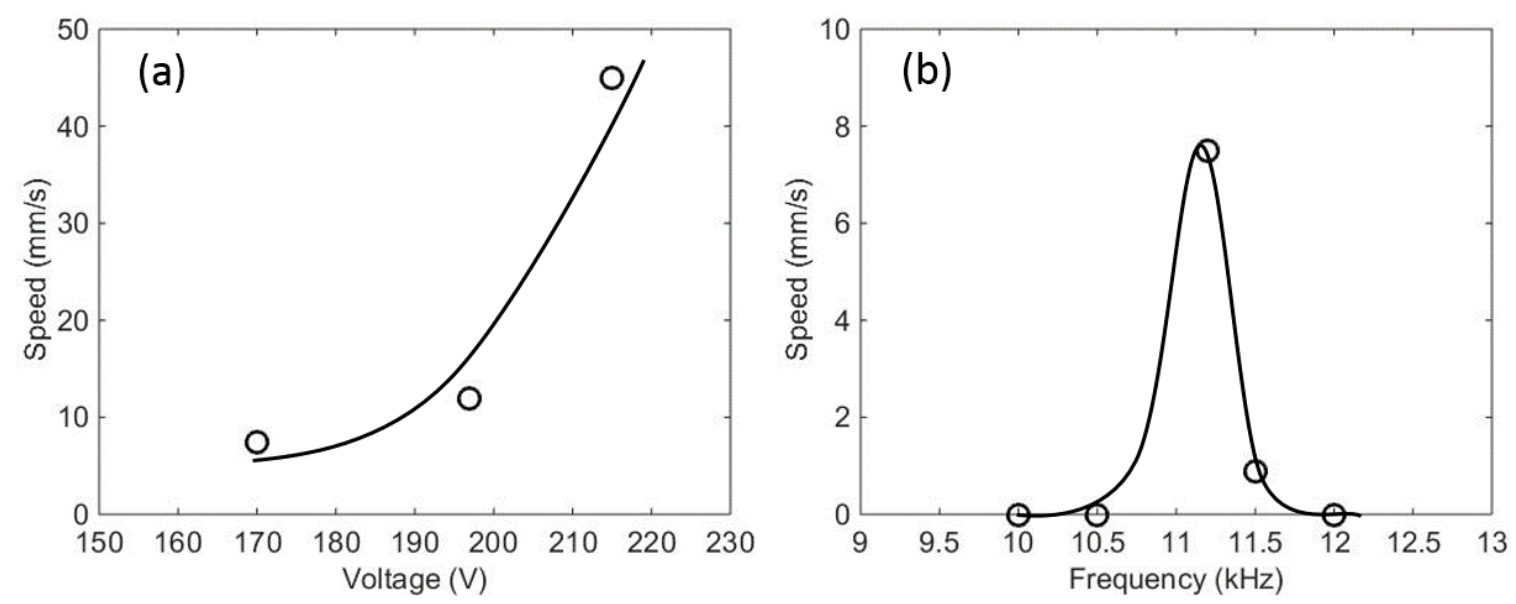

Figure 2.15. Propulsion speed vs. (a) applied voltage and (b) frequency.

To confirm and explain the reason for increasing propulsion speed, a hydrophone is used to measure the acoustic pressure inside the testing water tank with increasing voltage input to the piezoelectric actuator. The hydrophone sensor is always kept vertically and $1 \mathrm{~mm}$ above the bottom of the testing water tank. Due to the difference of acoustic pressure at different areas of the water tank, measurements are done at the four corners and the center area of the tank. Because of the fluctuation of the measurement results, multiple tests are done and the average values of reading are recorded, as shown in Table 1. 
Table 1. Acoustic pressure measurement by hydrophone at different input voltage for $11.2 \mathrm{kHz}$

\begin{tabular}{|c|c|c|c|c|c|c|c|c|c|c|c|}
\hline $\begin{array}{c}\sum_{\text {Qut }\left(\mathrm{V}_{p p}\right)} \\
\text { Input }\left(\mathrm{(}_{p \nmid \times}\right.\end{array}$ & center & $\begin{array}{c}\text { left } \\
\text { bottom }\end{array}$ & $\begin{array}{c}\text { right } \\
\text { bottom }\end{array}$ & $\begin{array}{c}\text { left } \\
\text { top }\end{array}$ & $\begin{array}{c}\text { right } \\
\text { top }\end{array}$ & voltage & center & $\begin{array}{c}\text { left } \\
\text { bottom }\end{array}$ & $\begin{array}{c}\text { right } \\
\text { bottom }\end{array}$ & $\begin{array}{c}\text { left } \\
\text { top }\end{array}$ & $\begin{array}{c}\text { right } \\
\text { top }\end{array}$ \\
\hline 77 & 39.7 & 25.3 & 59.3 & 37.3 & 38.3 & 190 & 106 & 75.7 & 165.3 & 97.3 & 118.3 \\
\hline 126 & 71.3 & 48 & 103 & 64 & 63 & 200 & 110 & 83.7 & 177 & 102.7 & 128.7 \\
\hline 165 & 90 & 63 & 143 & 86 & 95.7 & 210 & 120.3 & 92.7 & 186.3 & 106 & 135.7 \\
\hline 170 & 90.7 & 65.7 & 151.7 & 89.7 & 101.3 & 220 & 129.3 & 108.7 & 202.3 & 116.3 & 157 \\
\hline 180 & 97.7 & 70.7 & 159.7 & 93 & 108 & & & & & & \\
\hline
\end{tabular}

It is clear that as the input voltage to the piezoelectric actuator increases, the hydrophone output voltage is increased. The acoustic pressure could be calculated by the following equation

$$
P=\frac{V_{r m s, \text { out }}}{10^{-\frac{210}{20}}} \times 10^{-6}
$$

where $P$ is the absolute acoustic pressure and $V_{r m s, o u t}$ is the root mean square voltage output from the hydrophone. If we take the average value of all the five measuring spots and convert the hydrophone output into acoustic pressure, the result is shown in Figure 2.16 as below. 


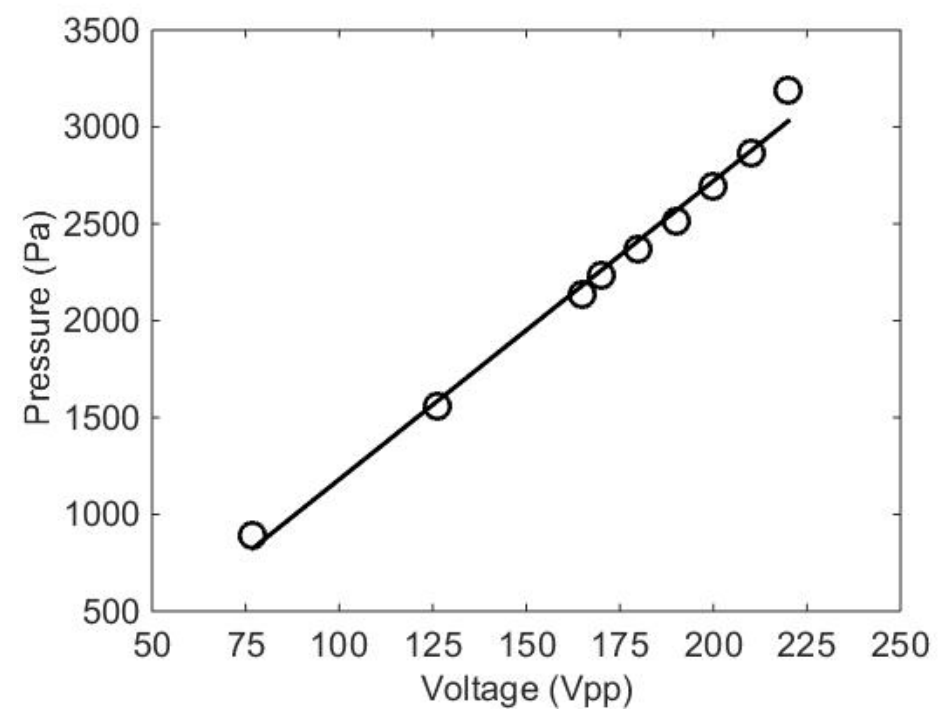

Figure 2.16. Input voltage vs. acoustic pressure inside the testing water tank.

A linear curve fitting is made to show the trend of data with the coefficient of determination of 0.90. It is obvious that higher input voltages generate stronger acoustic fields. The acoustic pressure amplitudes measured by the miniature reference hydrophone are 2.23, 2.52 and 2.87 $k P a$ for 170190 and $210 V_{p p}$, respectively. Therefore, it explains the reason for increasing propulsion speed in Figure 2.15 (a). Notice, the speed increasing trend in Figure 2.15 (a) is not exactly linear, possibly due to the measurement error caused by the stick-and-slip motions by friction with the wall of the tank, which might be caused by a small amount of data.

Figure 2.15 (b) shows the effect of the input frequency between $9 \mathrm{kHz}$ and $12 \mathrm{kHz}$ revealing a clear peak. For this frequency test, the voltage is fixed at $170 V_{p p}$. The maximum propulsion speed occurs at $11.2 \mathrm{kHz}$, which is about $7.5 \mathrm{~mm} / \mathrm{s}$ and the data is confirmed by the first data point in Figure 2.15 (a). When the driving frequency is even slightly shifted, the propulsion speed decreases rapidly. In many cases, the motions are uncertain (stick-and-slip motions) due mainly to friction. It is possible to turn on and turn off this propulsion by simply changing the 
frequency of acoustic input. In addition, if multiple bubbles with different resonances are built on the same chip, changing the input frequency allows us to activate only selected bubbles. This can be used for two-dimensional propelling and steering ${ }^{78}$.

The acoustic pressure with different frequencies is also tested to confirm the trend of the curve of Figure 2.15 (b), and the result is shown in Figure 2.17. Based on the previous acoustic pressure measurement, the acoustic field is not uniform inside the testing water tank depending on the area, therefore, for the accuracy, this time, the hydrophone probe is placed at the area where the device is propelled, instead of measuring the tank corners and center, and it is still kept vertically and $1 \mathrm{~mm}$ above the bottom of the tank bottom.

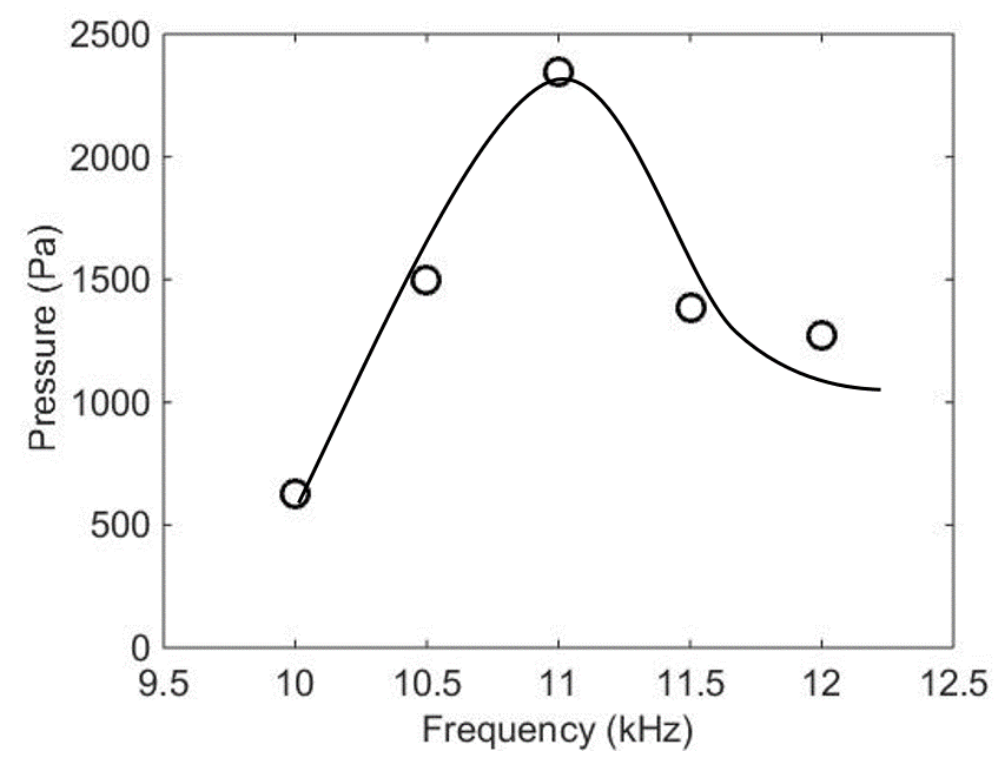

Figure 2.17. Input frequency vs. acoustic pressure inside the testing water tank.

It can be seen that there will be a peak acoustic pressure in the water tank near $11 \mathrm{kHz}$ that is the acoustic behavior of water tank itself instead of microbubbles. In fact, in both single-bubble 
device and arrayed device, the maximum oscillation and propulsion speed occur at $11.2 \mathrm{kHz}$, which is slightly different from the predicted frequency $(10.2 \mathrm{kHz})$ by Equation (8). This difference can be explained by the effect of the resonance behavior of the tank-water system. In other words, at a frequency of about $11 \mathrm{kHz}$, an acoustic wave can be transferred to the water environment more efficiently. Therefore, even though it is not exactly the bubble resonance, the oscillation amplitude is still larger than other frequencies due to the large acoustic pressure

amplitude. In addition, the loss (damping) mechanism ${ }^{17,70}$ in oscillation is also important to relate the acoustic pressure input to the oscillation amplitude, which has not been studied here.

\subsection{BI-DIRECTIONAL AND TWO-DIMENSIONAL MICRON SCALE ACOUSTIC BUBBLE PROPULSION}

Previously, it is shown that the parylene microswimmer could realize the underwater propulsion in one dimension with a relatively large speed up to 50 body lengths/second. The propulsion force is strong enough to carry a payload. In addition, the bubbles installed on turbo blades rotate the blades at $75 \mathrm{rpm}$. Some advantages of this acoustic excitation are (1) the structure of the swimmer is very simple. The main component is the one-end open micro tube filled with gas; (2) the swimmer is actuated wirelessly without any physical tethering; and (3) the external actuation system is neither costly nor bulky, possibly allowing us to utilize the existing ultrasound imaging/actuating system with minimal adjustment or amendment.

However, in order to become a more practical device, this microswimmer should be able to start from a certain position and swim in a selected direction to fulfill the navigation or medicine delivery in vivo. In other words, two- and even three-dimensional propulsion is required. 
Therefore, in this section, it describes how we achieve two-dimensional steering motions of acoustic bubble microswimmers. The key idea is to utilize the dependence of the resonance frequency on the microchannel (bubble) length, similarly occurring in many musical wind instruments. Multiple bubbles with various lengths are mounted on a microswimmer and aligned in different directions. When an acoustic field is applied to the bubbles, only frequency-matched bubbles are selectively activated and generate a strong propulsion force. Changing the frequency results in different selections of bubbles and thus propulsion in the different direction.

This part of work begins with device design and experimental setup. Previously, it was shown that the acoustic field reaching the bubbles and thus the frequency response of the bubbles are highly influenced by the associated experimental setup that also has its own resonance characteristics. For this reason, it is not safe to design devices by simply following the existing resonance Equation (8). Therefore, we first measure the frequency characteristics of the entire experimental setup. Based on this result, the bubble length is determined such that the bubble resonates in a particular frequency. Detailed flow fields and mechanism are studied using a 3-D CFD analysis with the commercial CFD package of ANSYS Fluent ${ }^{\circledR}$. Using carefully designed devices, we first achieve bi-directional linear and rotational motions and then two-dimensional steering propulsion even with payload carrying in a T-junction channel. All these are commandcontrolled by simply changing the acoustic frequency. The preliminary results of this frequencymatching method have been reported in the conferences ${ }^{79}$. Later, Ahmed et al. used this method to select and activate only desired bubbles out of a group of bubbles ${ }^{80}$. 


\subsubsection{Device Fabrication and Experimental Set-up}

The device fabrication process is exactly the same as the mentioned in the previous section 2.3.2.1. The only difference is that the two lengths of the microchannels are designed and fabricated: $450 \mu \mathrm{m}$ and $920 \mu \mathrm{m}$. The rectangular cross-section of all the microchannels has a height of $45 \mu \mathrm{m}$ and a width of $80 \mu \mathrm{m}$. Upon submerging the tubes, the sizes of created bubbles are between 270 and $300 \mu \mathrm{m}$ for the short tubes and between 760 and $820 \mu \mathrm{m}$ for the long tubes, depending on the condition of surface and opening profile. Note that the length of bubble was slightly reduced during the experiment due to bubble oscillation. To generate bi-directional (linear and rotational) and two-dimensional motions, multiple bubbles with different lengths can be individually aligned in multi-directions in the device. For bi-directional motion, the bubbles of two different lengths are aligned parallel to each other on the swimmer but the openings are placed near the edge of the swimmer. As a result, the openings of the short bubbles are opposite those of the long bubbles. For two-dimensional steering motions, the long bubbles are aligned orthogonally to the short bubbles.

For the experimental set-up, all of the equipment is the same as mentioned in section 2.3.2.2. Besides these equipment, a laser Doppler vibrometer (LDV, Micro System Analyzer Model MSA-400, Polytec, INC.) is used to measure the oscillation velocity of the tank bottom plate on which the microswimmer is located. 


\subsubsection{Acoustic Measurement}

The previous two-dimensional CFD simulation result in section 2.3.1 showed that the propulsion force $F$ is related to the amplitude $a$ and frequency $f$ of bubble oscillation as:

$$
F \cong 0.8 \rho A U^{2}=0.8 \rho A(a f)^{2}
$$

For a given oscillating frequency, the propulsion force is proportional to the square of oscillation amplitude. That is why it is important to maximize the oscillation amplitude to generate a strong propulsion force. In other words, the bubbles should oscillate in resonance. The resonance frequency was modeled in literature as the previous Equation (8).

However, this equation is only useful when the acoustic field strength reaching the bubbles is uniform over a wide range of the frequency. In reality, the strength of acoustic field exciting the bubbles is highly influenced by the resonance behaviors of the entire setup including the tank, medium, piezoelectric actuator and so on, although the input to the actuator is maintained constant as shown in previous Figure 2.17. This is because the above individual components have their own resonance behaviors. As a result, the experimental method is used to find the frequency to maximize the bubble oscillation amplitude.

\subsubsection{EFFECT OF WATER LEVEL}

It is very important to know the oscillation behavior of the entire testing setup filled with the medium (water). The oscillation behavior of the setup is examined for three different water levels in the tank. While the frequency of the acoustic field sweeps from $1 \mathrm{kHz}$ to $12 \mathrm{kHz}$, the oscillation velocity of the tank wall is measured using a LDV. The measurement location is near the center of the tank bottom plate on which microswimmers are examined in the following propulsion experiments. The result shown in Figure 2.18 reveals that the oscillating behavior of 
water tank bottom is strongly affected by the water level. This is mainly attributed to the change in the mass of the whole system. When the water level is $3 \mathrm{~cm}$, there are two distinct peaks at 6 $\mathrm{kHz}$ and $11 \mathrm{kHz}$ and it confirms the acoustic pressure measurement result in Figure 2.17. It can be reasonably assumed that this spectrum will be very similar to the actual acoustic input to the bubble since bending waves in the tank wall is the main component to generate acoustic waves reaching bubbles. In other words, the acoustic wave amplitude propagating to bubbles in water should be peaked at $6 \mathrm{kHz}$ and $11 \mathrm{kHz}$ when the water level is fixed at $3 \mathrm{~cm}$ and the input to the piezoelectric actuator is maintained. As a result, the amplitude of bubble oscillation would be highly increased at these two frequencies even if the acoustic frequency may not exactly fall in the resonance of bubble itself.

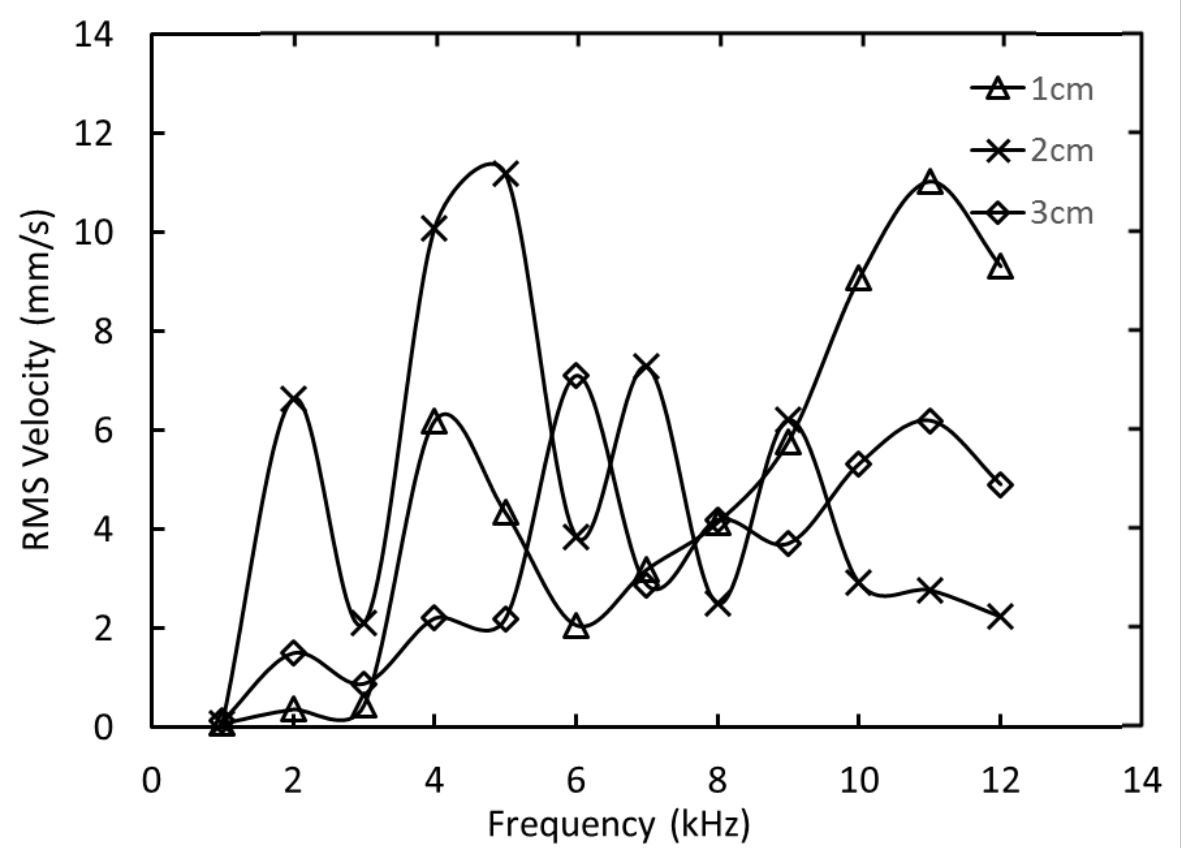

Figure 2.18. Oscillation velocity of the tank bottom at different frequency with different water depth. 
One may have a question that why the microswimmer in previous section 2.3.3 has the maximum speed at about $11 \mathrm{kHz}$ instead of $6 \mathrm{kHz}$. The answer is that the resonance frequency of the bubble (10.5 kHz, calculated by Equation (8)) is very close to $11 \mathrm{kHz}$ but far from $6 \mathrm{kHz}$. As a result, even though the acoustic wave amplitude (or acoustic pressure) is pretty strong at both of these two frequencies, the bubble will respond to $11 \mathrm{kHz}$ better. Consequently, in order to predict the spectrum behaviour of bubble oscillation (propulsion), all combined effects of the surrounding system need to be taken into account.

\subsubsection{EFFECT OF BUBBLE LENGTH}

In order to generate a two-dimensional steering propulsion, we need at least four bubbles whose openings face in four different directions: forward, backward, left and right. Ideally, the four bubbles need to be resonated independently. As a result, only selected bubbles generate propulsion when an applied acoustic field reaches all the bubbles. That is, the individual bubbles should resonate at different frequencies. Only the frequency-matched bubble generates a strong oscillation and propulsion while non-matched bubbles generate weak or negligible oscillation and propulsion. According to Equation (8), the bubble length is one of the main parameters to change the resonance frequency. We choose two different bubbles with length of $270 \mu \mathrm{m}$ and $820 \mu \mathrm{m}$ respectively in this experiment. We directly measure generated microstreaming for these two different bubbles (270 $\mu \mathrm{m}$ and $820 \mu \mathrm{m}$ ) while varying the frequency. The water level is fixed at $3 \mathrm{~cm}$ throughout the experiments. To measure the generated streaming speed, 2- $\mu m$-diameter microparticles are seeded in the water. Sequential snapshots are taken when the microparticles are pushed away by the bubbles. The speed is calculated by the particle displacement and the time interval between two different snapshots. 


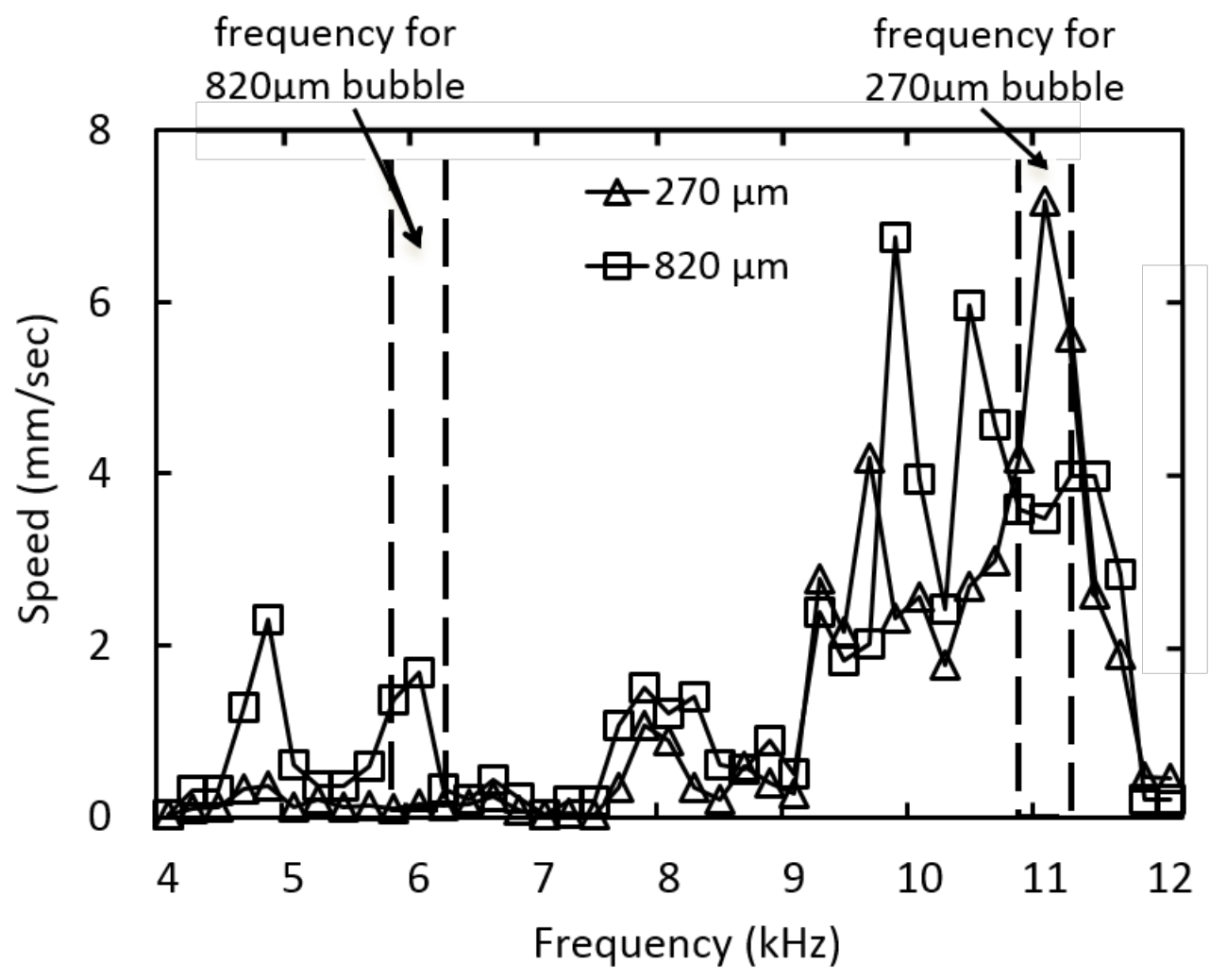

Figure 2.19. Streaming speed vs. frequency for $820 \mu m$ and $270 \mu m$ long bubbles at $150 V_{p p}$.

Figure 2.19 shows the generated microstreaming speed vs. the acoustic frequency for the two bubble lengths. Their frequency responses are quite different from each other. For example, at about $6 \mathrm{kHz}$, the $820 \mu \mathrm{m}$ bubble generates much stronger speed than the $270 \mu \mathrm{m}$ bubble while at about $11 \mathrm{kHz}$ the $270 \mu \mathrm{m}$ bubble generates stronger speed than the $820 \mu \mathrm{m}$ bubble. Note that these two frequencies are very close to the peak frequencies of tank oscillation as shown in Figure 2.18. Each peak frequency in the tank oscillation corresponds to each resonant frequency of the two bubbles. It confirms that the bubbles' acoustic behavior is strongly affected by the water tank. As a result, the long bubble resonates at $6 \mathrm{kHz}$ and the short bubble does at $11 \mathrm{kHz}$. These results are different from those predicted by Equation (8), which are $6.3 \mathrm{kHz}$ and $8.2 \mathrm{kHz}$ 
for the long and short bubbles respectively. This frequency discrepancy should be caused by the effect of the entire acoustic behavior. Based on these results, we select these two frequencies and two bubble lengths to generate bi-directional and two-dimensional propulsion in the following sections.

\subsubsection{Bi-directional Propulsion}

A bi-directional (back and forth) actuation is examined by using a microchannel arrayed device, which has six short microchannels with an averaged length of $450 \mu \mathrm{m}$ and three long microchannels with the averaged lengths of $920 \mu \mathrm{m}$ as shown in Figure 2.20. When the device is submerged in water, six short bubbles (average length $300 \mu \mathrm{m}$ ) and three long bubbles (average length is $760 \mu \mathrm{m}$ ) are formed. All the bubbles are aligned parallel to each other, but the openings of $450 \mu \mathrm{m}$ microchannels are opposite those of $920 \mu \mathrm{m}$ microchannels. Based on the results in Figure 2.19, there should be two bubble oscillation peaks, which are around 6 and $11 \mathrm{kHz}$. It is found that at $5.8 \mathrm{kHz}$ and $150 V_{p p}$ (acoustic pressure about $3 \mathrm{kPa}$ measured by a miniature hydrophone), propulsion by long bubbles $(760 \mu \mathrm{m})$ is strong enough to overcome all resistance forces including the propelling force generated by the short bubbles (300 $\mu \mathrm{m})$, water drag on the entire device and friction with the tank’s bottom surface. As a result, the device is propelled up as shown in Figure 2.20 (a), although the propulsion speed is slower than the previous onedimensional motion due mainly to the opposing force that is generated by the short bubbles. On the contrary, when the frequency is switched to $11.2 \mathrm{kHz}$, the propelling force by the short bubbles is dominant and pushes the device downward as shown in Figure 2.20 (b). This process could be repeated by simply switching the input frequency. The device consistently showed repeated back and forth motions regardless of the initial orientation of the device. 

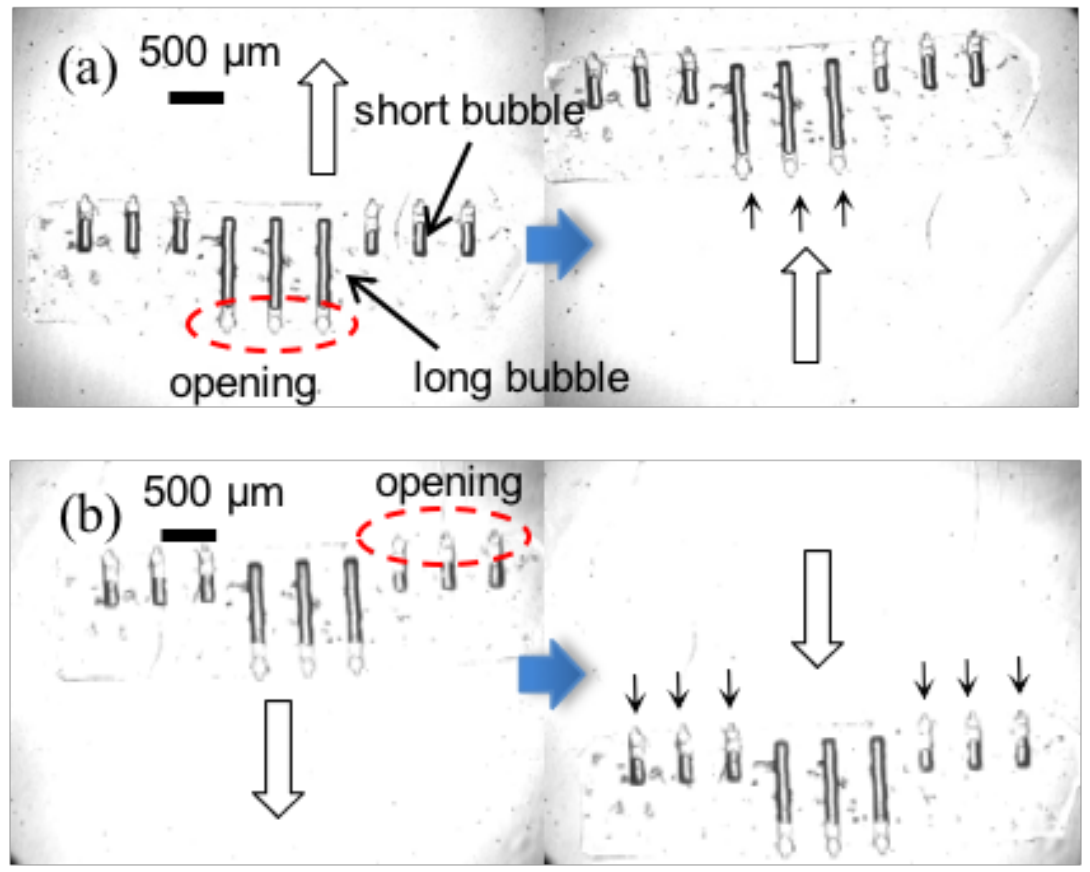

Figure 2.20. Bi-directional (back and forth) propulsion (bottom view) by (a) $820 \mu \mathrm{m}$ long bubbles at 5.8 $\mathrm{kHz}$ and (b) $270 \mu \mathrm{m}$ short bubbles at $11.2 \mathrm{kHz}$.

The oscillation amplitude for all nine bubbles is measured at both frequencies using a high speed camera, as shown in Table 2. It is clear that the short $(300 \mu m)$ bubbles have stronger oscillation at $11.2 \mathrm{kHz}$ than at $5.8 \mathrm{kHz}$ while the long bubbles $(760 \mu \mathrm{m})$ are stronger at $5.8 \mathrm{kHz}$ than at $11.2 \mathrm{kHz}$. This is consistent with the micro streaming velocity measurement in Figure 2.19 and proves the concept of activating the frequency-matched bubbles by changing input acoustic frequency. 
Table 2. Oscillation amplitude for six short and three long bubbles at $5.80 \mathrm{kHz}$ and $11.2 \mathrm{kHz}$.

\begin{tabular}{|c|c|c|c|c|c|c|c|}
\hline & \multicolumn{7}{|c|}{ Short Bubble $(270-\mu \mathrm{m})$ Oscillation Amplitude $(\mu \mathrm{m})$} \\
\hline & 1 & 2 & 3 & 4 & 5 & 6 & average \\
\hline $5.80 \mathrm{kHz}$ & 22.9 & 23.3 & 28.2 & 15.8 & 27.4 & 29.3 & 24.5 \\
\hline \multirow[t]{3}{*}{$11.20 \mathrm{kHz}$} & 37.9 & 24.0 & 30.8 & 29.2 & 30.7 & 33.4 & 31.0 \\
\hline & \multicolumn{7}{|c|}{ Long Bubble $(820-\mu \mathrm{m})$ Oscillation Amplitude $(\mu \mathrm{m})$} \\
\hline & \multicolumn{2}{|c|}{1} & \multicolumn{2}{|l|}{2} & 3 & \multicolumn{2}{|c|}{ average } \\
\hline $5.80 \mathrm{kHz}$ & \multicolumn{2}{|c|}{60.6} & \multicolumn{2}{|c|}{66.8} & 61.3 & \multicolumn{2}{|c|}{62.9} \\
\hline $11.20 \mathrm{kHz}$ & \multicolumn{2}{|c|}{28.8} & \multicolumn{2}{|c|}{23.8} & 22.9 & \multicolumn{2}{|c|}{25.2} \\
\hline
\end{tabular}

To further investigate the total net propelling forces, three-dimensional numerical simulations are performed using the commercial CFD (Computational Fluid Dynamics) package of ANSYS Fluent ${ }^{\circledR}$. To reduce computation time, simulations are carried out for a representative single long and short bubble, respectively, rather than for all nine bubbles on the testing devices. The oscillation amplitudes of each simulated bubble are assigned the same as the averaged amplitude shown in Table 2. Based on the oscillation amplitude and tube dimensions, the corresponding Reynolds numbers are calculated using Equation (3) in previous section 2.3.1. Note that the cross section of the microchannel is square, so the hydraulic radius is used.

The computational domain for the short bubble is extended to 5 times the microchannel width laterally, 10 times the height vertically and 20 times the length longitudinally. The longitudinal computation dimension is set much larger than the other two dimensions because the change in 
propulsion induced flow mainly occurs in the longitudinal direction. Two different configurations are simulated for the long and short bubbles: (1) the tube placed on the bottom wall and (2) the tube placed in the middle between the water surface and bottom wall. The boundary conditions are that the bottom and side walls are the no-slip condition while the top surface is set to be the atmospheric pressure condition. The interaction between water and air at the tank top interface is neglected for simplicity: the interface is assumed horizontally flat. In addition, it is assumed that the flow is symmetric with respect to the vertical symmetric line of the microchannel. Only half of the full domain with respect to the vertical symmetric line is simulated. Furthermore, although the flow near the tube opening is oscillatory, simulations are performed for two steady-state cases of intake and discharge with a constant flow rate, which are later averaged to approximate the oscillatory flow.

The simulated streamlines are shown in Figure 2.21 for the short microchannel. It can be seen that when the microchannel intakes the fluid, in other words the bubble shrinks, the fluid comes into the tube opening from all directions as shown in Figure 2.21 (a) and (c). In contrast, when the microchannel discharges the fluid (the bubble expands), the discharged stream is in the longitudinal direction as in Figure 2.21 (b) and (d). The difference of the fluid patterns generates a net force (propelling force) on the tube when the intake and discharge alternate. 


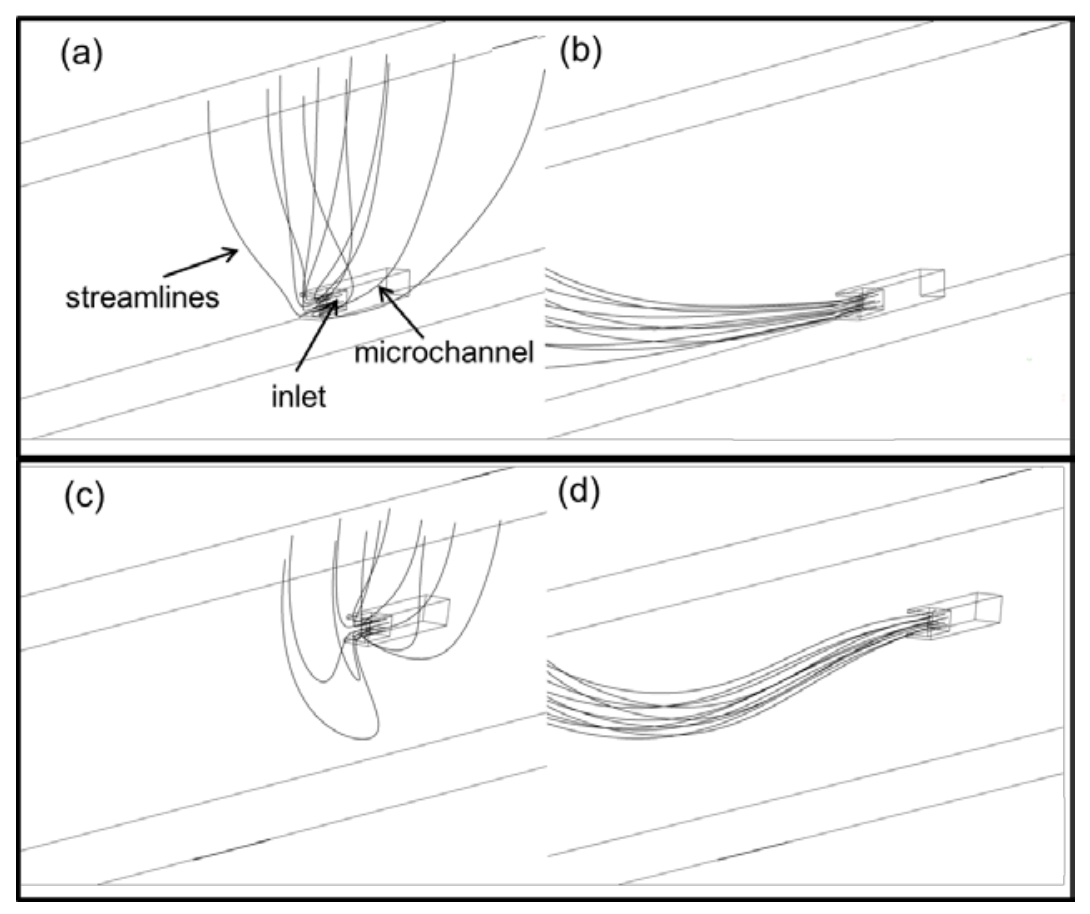

Figure 2.21. Simulated streamlines of the microchannel when placed (a) on the bottom of channel and intakes, (b) on the bottom of channel and discharges, (c) in the middle of the channel and intakes and (d) in the middle of the channel and discharges.

Figure 2.22 (a) shows the net force on the microchannel vs. the Reynolds number when it is put on the bottom of the tank and the intake and discharge results are summed up. The net force is proportional to the Reynolds number. In this simulation, all of the short/long tubes are placed on the bottom of the testing water tank. The simulation results also confirm the bi-directional propulsion in the above experiment. At $5.8 \mathrm{kHz}$, the long bubble generates an average propulsion force of $4.53 \mu N$. As a result, the total force from 3 long bubbles on the device is $4.53 \times 3=$ $13.59 \mu N$. On the other hand, the short bubble generates $0.67 \mu N$ that results in $0.67 \times 6=$ $4.02 \mu N$ in the total force. Therefore, the net propulsion force on the device is $13.59-4.02=$ $9.57 \mu N$, even though the number of long bubbles is 3 less than that of short bubbles. Similar 
calculations can be made for $11.2 \mathrm{kHz}$. The total force generated by 6 short bubbles is $4.08 \times 6=$ $24.48 \mu N$ while the force generated by 3 long bubbles is $2.71 \times 3=8.13 \mu N$. As a result, the net force on the device is $24.48-8.13=16.35 \mu N$.
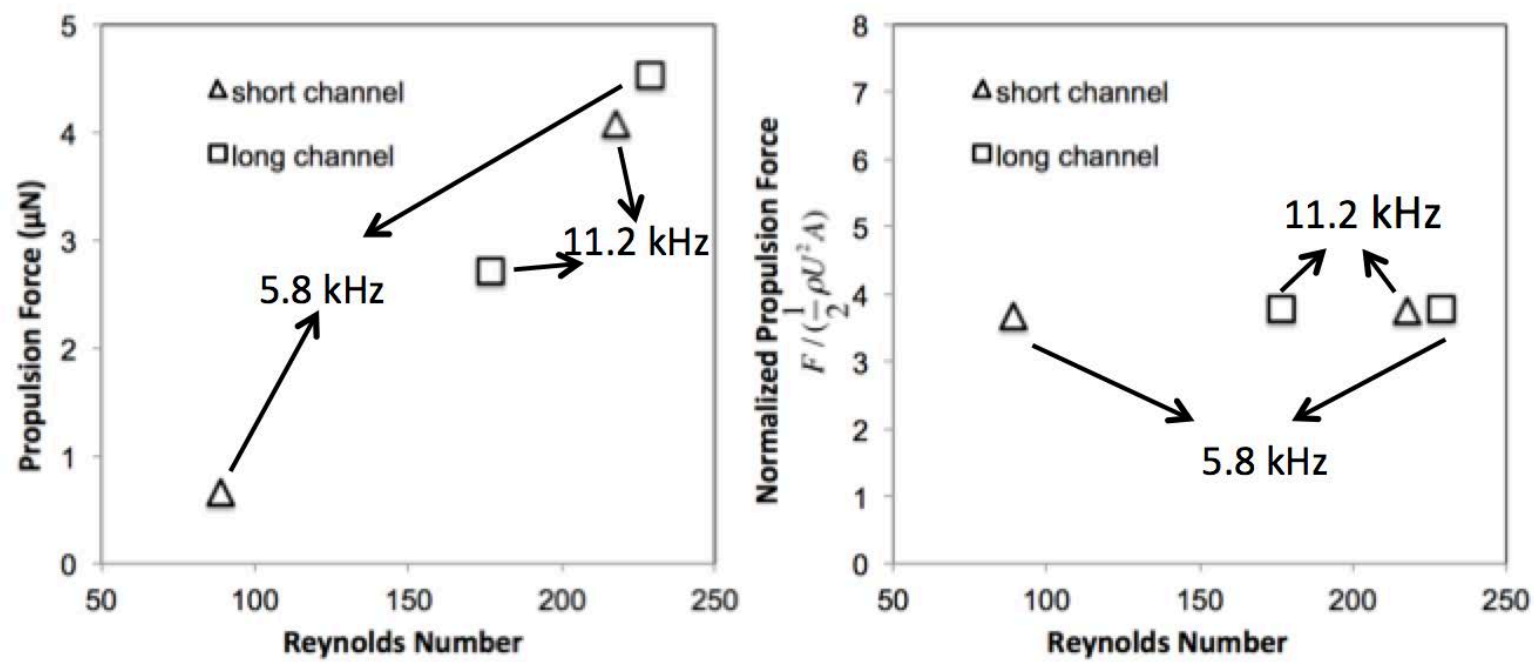

Figure 2.22. Numerical results of the propulsion force for all the short and long bubbles at (a) $5.8 \mathrm{kHz}$ and (b) $11.2 \mathrm{kHz}$.

Note that although the difference in the oscillation amplitude between the short and long bubbles is not very large at $11.2 \mathrm{kHz}$, compared to at $5.8 \mathrm{kHz}$, the net total force is still strong enough. This can be explained by Equation (7) that the propelling force is proportional to the square of the frequency. At a high frequency, even a small difference in oscillation amplitude can lead to a large propelling force.

When the microchannels trap air bubbles, the final bubble length varies from one bubble to another even in the same device since it is sensitive to the shape of the tube opening and roughness. In the above simulations, the bubble length is chosen for the average length of the 
multiple bubbles in the testing devices. To investigate the effect of bubble length on the propulsion force, a simulation for $277 \mu \mathrm{m}$ bubble length is performed as opposed to $300 \mu \mathrm{m}$ in the above simulation (about 10\% change). First, when the short microchannel is placed on the bottom of the water tank bottom, the simulation result shows that the generated propulsion force is $4.11 \mu N$ at $11.2 \mathrm{kHz}$ which is close to the previous value of $4.08 \mu N$ (less than $1 \%$ ). That is, the generated force does not depend on the bubble length very much. The oscillating speed of the bubble interface is the most important parameter to determine the propelling force. Besides the propulsion force in axial direction, we found that the vertical force of $0.64 \mu \mathrm{N}$ is generated towards the tank bottom, even though it is much weaker than that in the axial direction. This small vertical force is caused by the asymmetric fluid fields above the device plane and below the plane in the vertical direction. The tank bottom below the device acts as a blockage to the flow while the top space of the device is open.

The flow by the same short microchannels is also simulated when it is placed on the middle plane between the tank bottom and top free surface. The result shows that propulsion force is 4.18 $\mu N$, which is very similar $(<2 \%)$ to being placed on the bottom. The vertical force component is reduced to $0.12 \mu N$. This is because the bottom wall effect is reduced due to enlarged distance between the bubble and bottom wall, therefore the fluid could also develop under the device. This additional streaming makes a little contribution on the asymmetric fluid patterns during water intake and discharge in the axial direction. As a result, the propulsion force becomes $0.07 \mu N$ more compared with the device put on the bottom. On the other hand, the streaming below the device plane also reduced the asymmetric in the vertical direction. Therefore, the vertical force is reduced. Overall, the generated propulsion force is mainly in the 
axial direction and the vertical component is very small regardless of the proximity to the wall. That is, the bubble swimmer can be strongly propelled in the axial direction.

As shown in Figure 2.22 (b), the computed propulsion force can be normalized by the momentum flux $\left(\rho U^{2} A / 2\right)$ for all the simulated bubbles, which is almost constant in the entire range of the $R e$. The propulsion force can be approximated based on this behavior:

$$
F \cong 1.88 \rho A U^{2}=1.88 \rho A(a f)^{2}
$$

where $U$ is the inlet velocity. The standard deviation for the coefficient in Equation (11) is 0.05 . The constant trend of the normalized propulsion force illustrates that as long as bubble oscillation amplitude and acoustic input frequency are fixed, the generated propulsion force will not change a lot with the bubble or microchannel configuration. Note that the coefficient for three-dimensional simulation is 1.88 which is larger thant the two-dimensional simulation 0.88 . The reason is that for two-dimensional simulation, there is only one inner wall of the microchannel which will dominate the propulsion force. However, for the three-dimensional case, there are three inner walls, therefore the force generation will be totally different.

Multiple bubbles of different length can also generate bi-directional rotations. As shown in Figure 2.23, two $270 \mu \mathrm{m}$ microchannels and two $820 \mu \mathrm{m}$ microchannels are all aligned parallel to each other at the four corners of a rectangular device platform. A pair of microchannels of the same length is placed diagonally with their openings opposite. The openings of all four microchannels face outward. The bubbles of the same length would generate a couple (torque). The center of the device is punctured by an optical fiber that is anchored at the bottom of the tank. When the acoustic field is applied at $11.0 \mathrm{kHz}\left(170 \mathrm{~V}_{p p}\right)$, the torque generated by the short bubble pair is greater than that generated by the long bubble pair. As a result, the device rotates clockwise as indicated by the white arrow in Figure 2.23 (a). The rotational direction is easily 
reversed by simply switching the frequency to $5.8 \mathrm{kHz}$ as shown in Figure 2.23 (b). The long bubble pair generates a dominant torque responsible for the counterclockwise rotation.
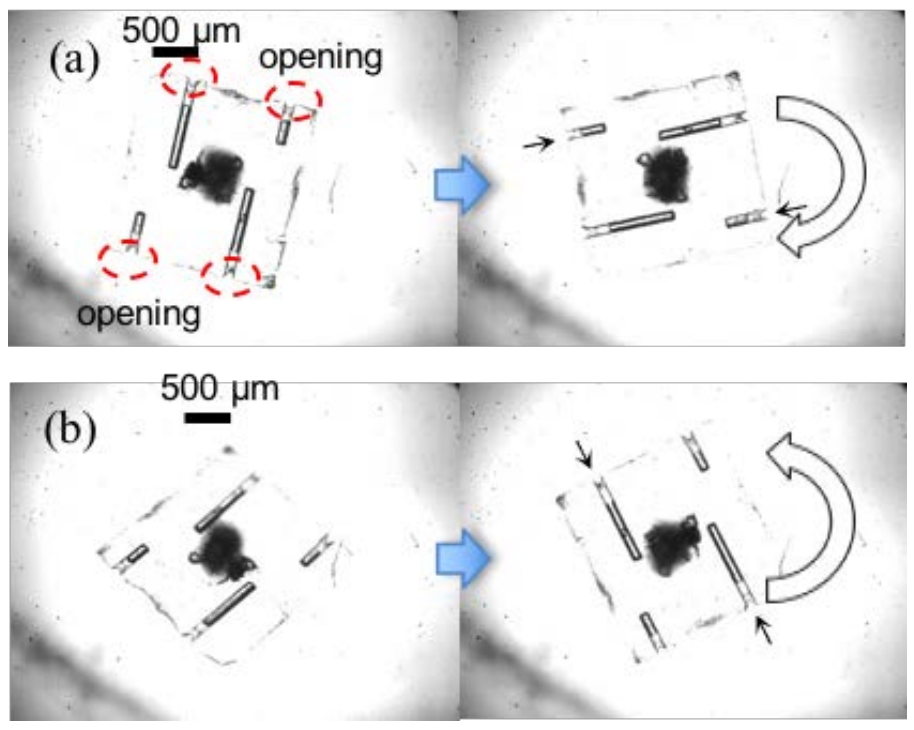

Figure 2.23. Rotation (bottom view) (a) clockwise rotation by $270 \mu \mathrm{m}$ long bubbles at $11.0 \mathrm{kHz}$, (b) counterclockwise rotation by $820 \mu \mathrm{m}$ bubbles at $5.8 \mathrm{kHz}$ and (c) bubble turbo-motor at $3.8 \mathrm{kHz}$.

\subsubsection{Two-dimensional Propulsion}

Based on the previous experiments, multiple bubbles are used to steer the device. Multiple bubbles are used to steer the device on a two-dimensional plane. Three short channels (270 $\mu \mathrm{m}$ long) and three long channels (820 $\mu \mathrm{m}$ long) are orthogonally aligned on a device in Figure 2.24. The switch of acoustic frequency could activate different bubbles to make the device steering and choose the propulsion direction as needed. 


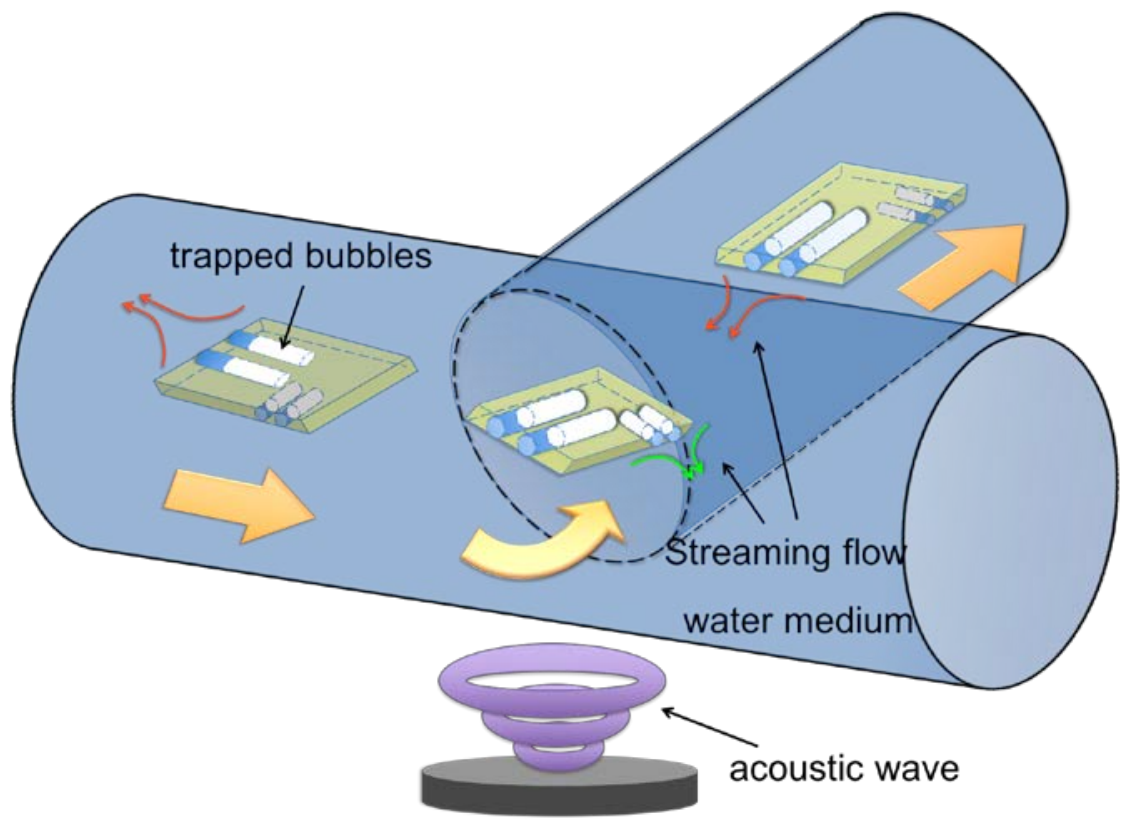

Figure 2.24. Two-dimensional motion device with three $820 \mu \mathrm{m}$ and three $270 \mu \mathrm{m}$ bubbles.

A square plastic film $\left(400 \times 325 \times 80 \mu m^{3}\right)$ is also attached to the device by a copper wire of $30 \mu m$ in diameter to examine the ability of payload carrying. The input signal is given at the frequencies of $5.8 \mathrm{kHz}$ and $11.2 \mathrm{kHz}$, respectively. The voltage is increased to $220 V_{p p}$ to overcome the additional drag that might be caused by the payload. When the frequency is set at $5.8 \mathrm{kHz}$, the device is propelled downward along a nearly linear path by the long bubbles as shown in Figure 2.25 (a). Due to non-uniform friction or the propelling force generated by the oscillation of the short bubbles, the device is slightly off the linear path. The propulsion in the perpendicular direction is achieved by switching the frequency to $11.2 \mathrm{kHz}$. The short bubbles are strongly excited and mainly responsible for the propulsion as shown in Figure 2.25 (b). Note that the motion is linear, not rotational. Since the three short bubbles are placed off the center of the device, they are supposed to generate both counterclockwise rotation and linear motion. 
However, due to the additional friction by the attached load, the generated torque is almost cancelled out. As a result, the almost linear motion is generated. When a two-frequency superposed signal (5.8 $\mathrm{kHz}$ and $11.2 \mathrm{kHz} 280 \mathrm{~V}_{p p}$ ) is applied, the device is propelled in the direction of the resultant of two major comparable forces generated by the short and long bubbles and other frictional forces, as shown in Figure 2.25 (c).
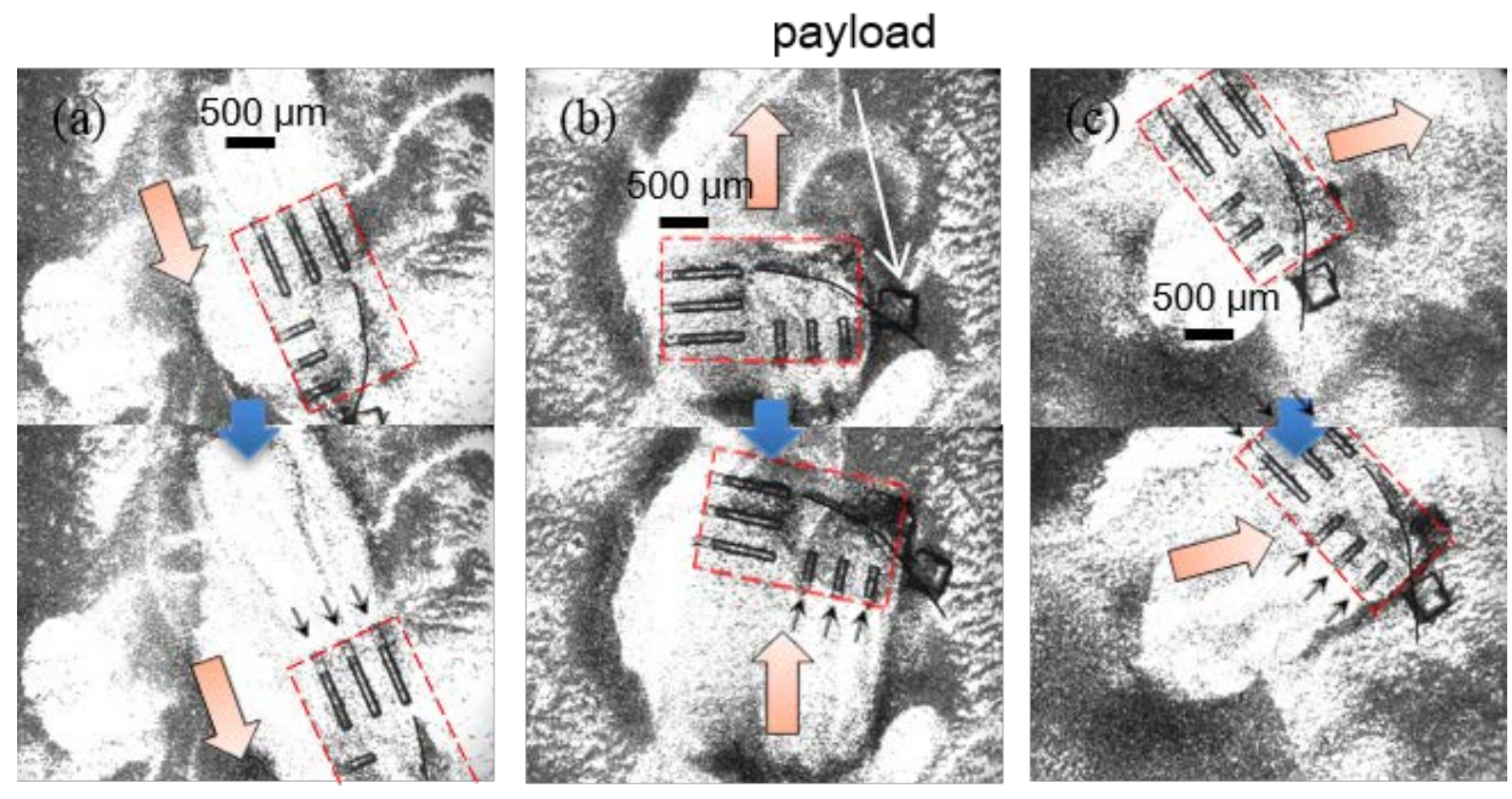

Figure 2.25. 2-D motions (a) the long bubbles push, (b) the short bubbles push and (c) all the bubbles push under superposed acoustic field of the two frequencies (particles were seeded for flow visualization).

The similar 2-D propulsion is carried out in a T-junction channel (perpendicularly formed by two $2 \mathrm{~mm}$ wide channels) to simulate navigation in a blood vessel junction. The acoustic signal of $6.0 \mathrm{kHz}\left(370 V_{p p}\right)$ is applied first where the long bubbles push the device downward entering the T-junction as shown in Figure 2.26 (a) and (b). Then, the acoustic frequency is switched to 
$11.0 \mathrm{kHz}\left(210 V_{p p}\right)$ such that the short bubbles are excited and generate a clockwise rotation in Figure 2.26 (c) and (d). As a result, the device is well aligned in the horizontal channel. Finally, the $6.0 \mathrm{kHz}\left(370 V_{p p}\right)$ signal is applied again and the long bubbles generate a dominant propulsion force. The device moves left along the horizontal channel and leaves the T-junction Figure 2.26 (e) and (f).

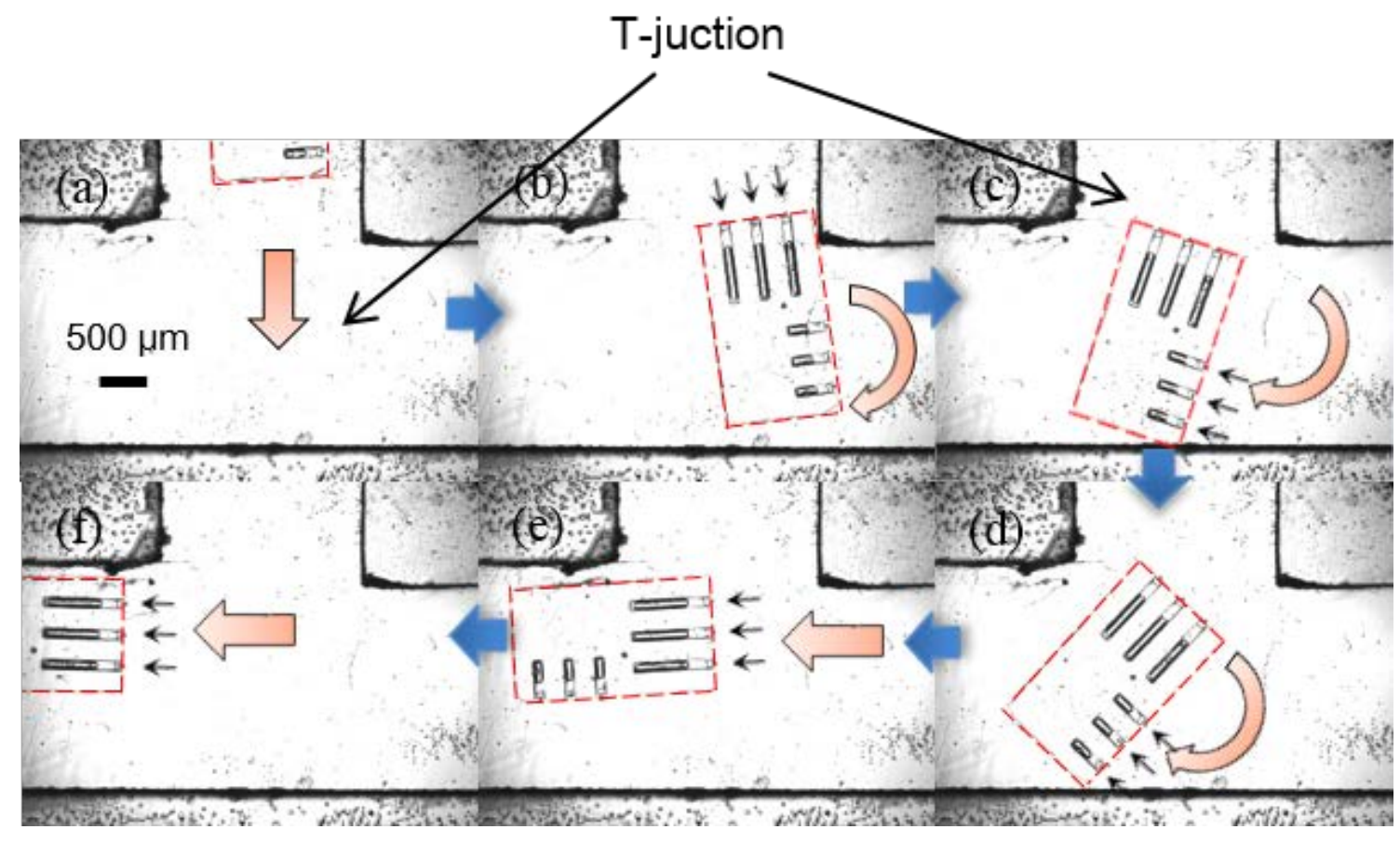

Figure 2.26. Steering capability in a T-junction of channel by $820 \mu \mathrm{m}$ and $270 \mu \mathrm{m}$ bubbles without tracking particles (a), (b) moving in (c), (d) counter-clockwise rotation and (e), (f) moving out.

A right turning and straight swimming is also demonstrated in the same T-junction by turning over the microswimmer upside down. This time, the $2 \mu \mathrm{m}$ tracking particles are added into the Tjunction to show the microstreaming generated by acoustic bubble oscillation. The input acoustic signal is exactly the same as previous testing which begins with $6.0 \mathrm{kHz}\left(370 V_{p p}\right)$ to enter the T- 
junction. Then, $11.0 \mathrm{kHz}\left(210 V_{p p}\right)$ is used to generate a counter-clockwise rotation. At last, the $6.0 \mathrm{kHz}\left(370 V_{p p}\right)$ is applied to swim out this T-junction area. The result shows good propulsion direction control ability.

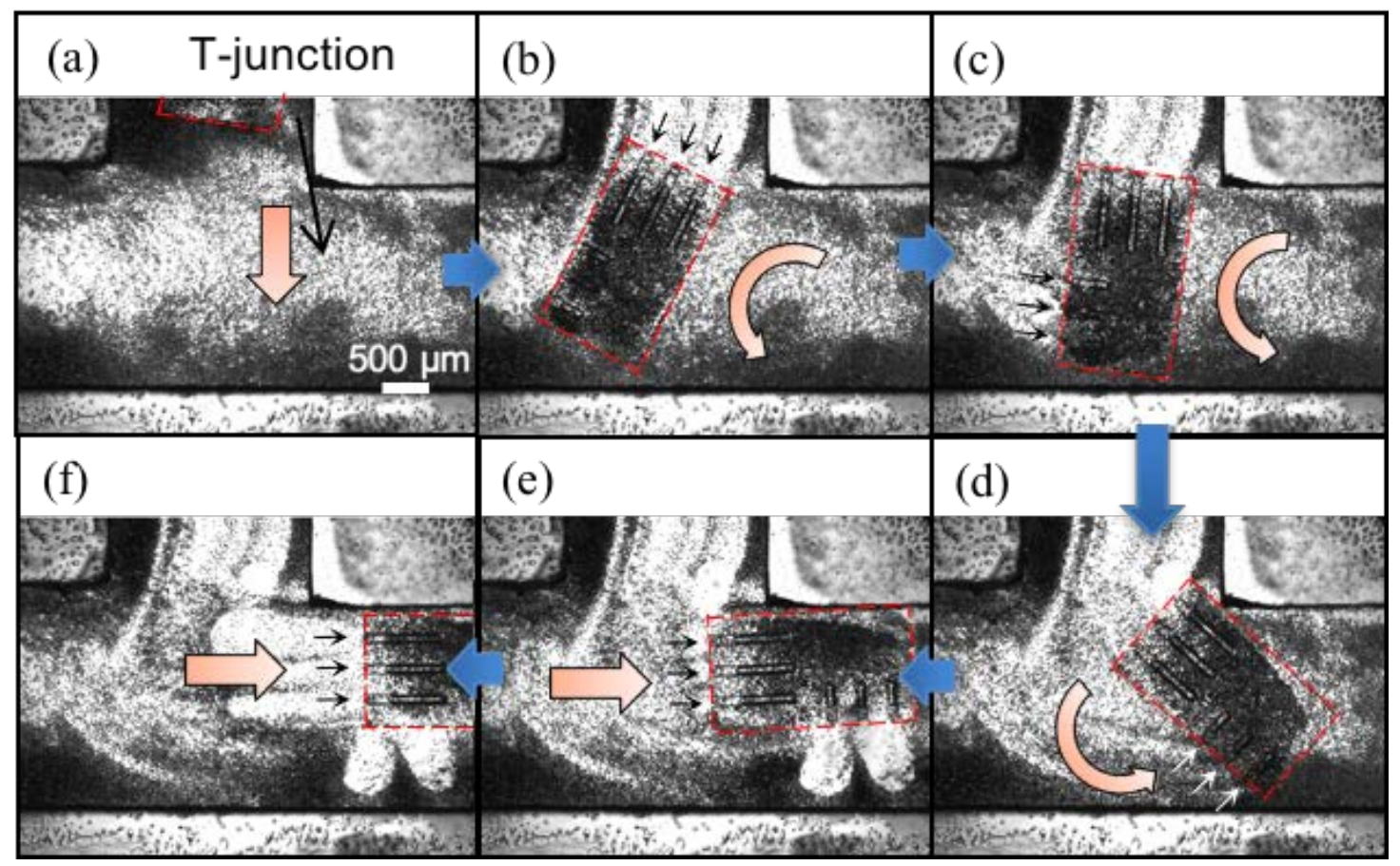

Figure 2.27. Steering capability in a T-junction of channel by $820 \mu m$ and $270 \mu m$ bubbles with tracking particles (a), (b) moving in (c), (d) clockwise rotation and (e), (f) moving out.

On the other hand, the tracking particles show the dominant force in each step of this motion. In the first step, from Figure 2.27 (a) to (b), the longer bubbles are responsible for the translation motion, therefore, most of the particles are ejected from the opening of the long channel in the axial direction. As a result, the empty region is formed behind the device near the long channel. On the contrary, almost no clear empty region is formed near the short channel in Figure 2.27 (a) and (b). In the second step, from Figure 2.27 (c) and (d) shorter bubbles are dominant for the 
rotation, and empty regions are forming near the opening of the short channel. There is no new clear empty region formed near the opening of long channel. In the third step, force generated by short bubbles becomes smaller and long bubbles work again from Figure 2.27 (e) to (f). The two empty regions formed by short bubbles microstreaming stay there and new empty regions formed by longer bubbles come out.

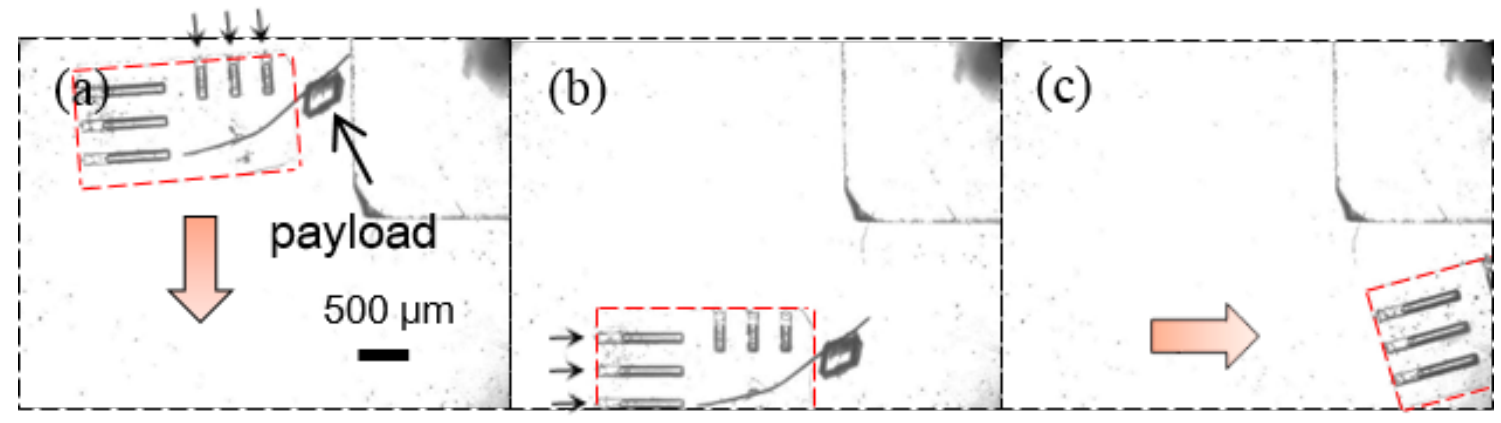

Figure 2.28. Steering capability in a T-junction of channel by $820 \mu m$ and $270 \mu m$ bubbles with load with (a) moving in, (b) switching frequency and (c) moving out.

A similar operation is made in the T-junction but carrying a payload as shown in Figure 2.28 (a) to (c). The device carries the payload moving downward first by the short bubbles and then moving to the right by the long bubbles. Again note that the motion generated by the short bubbles is linear, not rotational, possibly because the rotation torque is balanced by the payload.

In conclusion, the concept for underwater propulsion by micron sized acoustic bubbles is further developed based on previous section 2.3. The bubbles' size, shape and arrangement are defined by the hydrophobic parylene microchannels made through microfabrication. This type of microswimmer is propelled by the oscillation and generated microstreaming flow. In order to maximize bubble oscillation amplitude, the laser Doppler vibrometer (LDV) is used to get the 
testing water tank acoustic behavior in the frequency range from $1 \mathrm{kHz}$ to $12 \mathrm{kHz}$ with different water depths of 1,2 and $3 \mathrm{~cm}$. The result shows the two acoustic peaks at $6 \mathrm{kHz}$ and $11 \mathrm{kHz}$ with water depth of $3 \mathrm{~cm}$. Based on this measurement result, two different lengths of bubbles are used in order to change the propulsion directions and steer the micro device on a two-dimensional plane. The long and short bubbles could resonate and generate strongest microstreaming at 5.8 $\mathrm{kHz}$ and $11 \mathrm{kHz}$ separately. Consequently, when the openings of long bubbles are aligned opposite those of short bubbles the bi-directional linear propulsion is achieved by simply changing the frequency between 5.8 and $11 \mathrm{kHz}$. The bubbles of these lengths are installed in a rectangular device to generate a torque. Similarly, by switching the frequency, the direction of rotation can be easily reversed between clockwise and counterclockwise. For two-dimensional steering, the short bubbles are aligned perpendicular to the long bubbles. Using this scheme, we successfully demonstrate that the device navigates through a T-junction in a channel with/without carrying a payload. During the navigation, the frequency is only the control input to select and resonate targeted bubbles. All these operations are performed by a single piezoelectric actuator. A potential application of this device may be microswimmers that navigate inside the human body to perform various missions such as bio-sensing, drug delivery, and micro surgery. 


\subsection{BIODEGRADABLE DEVICE}

In the previous sections, it is shown that a micro device based on a parylene structure could realize both one- and two-dimensional motions, including one-dimensional motion and twodimensional steering. It also has the ability to carry a payload. However, parylene is still not a biodegradable material, which means it cannot be broken down into the base components of carbon, hydrogen and oxygen by microorganisms. In other words, if it is used in vivo, the device will stay intact inside of the body after finishing its function. Therefore, to realize the desired bio-application, such as navigation through blood vessels and drug delivery research will be focused mainly on biodegradable device fabrication in this section.

\subsection{BIODEGRADABLE DEVICE FABRICATION}

A biomaterial has to be biocompatible which means that they perform their function with an appropriate host response ${ }^{81}$. However, in order to complete the in vivo job, such as drug delivery, the material has to be degradable inside body.

To make the material appropriate to be used clinically in vivo, the material must have the following properties, (1) not evoke a sustained inflammatory response; (2) possess a degradation time coinciding with their function; (3) have appropriate mechanical properties for their intended use; (4) produce non-toxic degradation products that can be readily reabsorbed or excreted; and 
(5) include appropriate permeability and process ability for designed application ${ }^{82}$. In other words, besides the suitable mechanical properties and biocompatibility, the material also requires degradation within clinically reasonable time periods ${ }^{83}$.

In all of the previous underwater propulsion experiments, the micro devices are fabricated from parylene which is a biocompatible material. However, if this microswimmer will be used in vivo in the future, the device itself has to be biodegradable in the blood environment. According to the different purposes, the device could be divided into two categories, long-term and shortterm use. In this paper, long-term means several months or even several years while short-term means several hours or several days. In the following sections, both long-term and short-term microswimmer design, fabrication, and experiments will be demonstrated.

\subsubsection{Long-term Biodegradable Device}

To realize long-term biodegradation, polymer is always one of the best choices. First of all, the density of polymer is not far from water, such as parylene. As a result, it could minimize the friction between the micro device and the swimming channel. Secondly, polymer is transparent, which makes it easier to observe bubble oscillation inside the device. Thirdly, polymer has a relatively low biodegradation rate which makes the device able to survive for a relatively long time underwater.

Table 3 shows the properties of several commonly used different kinds of biodegradable polymers $^{84,85}$, including the prices (from Sigma-Aldrich Co.). 
Table 3. Property and price for biodegradable polymers.

\begin{tabular}{|c|c|c|c|}
\hline Polymer Name & Degradation Time & Melting Point $\left({ }^{\circ} \mathrm{C}\right)$ & Price $(\$ / g)$ \\
\hline Polyglycolide (PGA) & 6-12 months & $225-230$ & $\sim 330$ \\
\hline \multirow{3}{*}{ Polylactide (PLA) } & $\begin{array}{l}\text { poly(L-lactic acid) } \\
\text { (PLLA) } \\
\text { High molecular weight }>5 \\
\text { years }\end{array}$ & 175 & \multirow{3}{*}{$\sim 200$} \\
\hline & $\begin{array}{l}\operatorname{poly(D-lactic~acid)} \\
\text { (PDLA) < } 6 \text { months }\end{array}$ & $264-350$ & \\
\hline & $\begin{array}{l}\text { poly(D,L-lactic acid) } \\
\text { (PDLLA) < } 6 \text { months }\end{array}$ & amorphous & \\
\hline \multirow{3}{*}{ Poly(lactide-co-glycolide) } & $\begin{array}{l}\text { 50:50 PLGA: } \\
\text { 1-2 months }\end{array}$ & amorphous & \multirow{3}{*}{$\sim 300-400$} \\
\hline & $\begin{array}{l}\text { 75:25 PLGA: } \\
\text { 4-5 months }\end{array}$ & amorphous & \\
\hline & $\begin{array}{l}\text { 85:15 PLGA: } \\
\text { 5-6 months }\end{array}$ & amorphous & \\
\hline Polycaprolactone (PCL) & 2-3 years & $58-63$ & $<10$ \\
\hline
\end{tabular}


From Table 3, it is clear that polycaprolactone (PCL) is one of the best biodegradable polymers, which could be used to fabricate the microswimmer for longer-term use. Because it was already proven that a parylene microswimmer could be successfully propelled under water in previous sections 2.3 and 2.4, the properties of PCL and parylene are compared in Table $4^{86}$ to estimate the possibility to be used for device fabrication.

Table 4. Physical properties of PCL and parylene C.

\begin{tabular}{|c|c|c|}
\hline & PCL & parylene C \\
\hline Density $\left(\mathrm{g} / \mathrm{cm}^{3}\right)$ & 1.1 & 1.289 \\
\hline Sound Speed $(\mathrm{m} / \mathrm{s})$ & 3,300 & 2,200 \\
\hline Young's Modulus $(\mathrm{GPa})$ & 1.2 & 3.2 \\
\hline Contact Angle $\left(^{\circ}\right)$ & 80 & 120 \\
\hline
\end{tabular}

The similar densities of PCL and parylene C means the frictions to bottom of the tank are not too different when the device is submerged in water. Similar products of density and sound speed means similar acoustic impedance between PCL and parylene C. Smaller Young's Modulus means the strength of microchannel might be reduced, however, it could be improved by increasing the thickness of the microchannel wall if necessary. The contact angle of PCL is 
smaller than parylene $\mathrm{C}$, but it is still not quite hydrophilic. It makes it possible to trap the air bubble inside of the PCL microchannel.

Steedman et al. illustrated a successful PCL coating process after a normal PDMS modeling process based on SU-8 photoresist. The PCL is dissolved in 2,2,2-trifluoroethanol at $65{ }^{\circ} \mathrm{C}$ for 3 hours with the concentration of $0.1 \mathrm{~g} / \mathrm{mL}$. The thickness of PCL film could reach about $5 \mu \mathrm{m}$ with a spin speed of $1,500 \mathrm{rpm}^{87}$. In addition, Simon et al. showed the PCL film fabrication process by spin coating the PCL dissolved tetrachloroethane solution on glass substrate ${ }^{88}$. By controlling the concentration of PCL solution (from 0.2 to $1.6 \mathrm{~g} / \mathrm{mL}$ ) and the spin speed (from 1000 to $6000 \mathrm{rpm}$ ), the thickness of PCL film could vary from less than $0.5 \mu \mathrm{m}$ to $6 \mu \mathrm{m}$. Nagstrup demonstrated a successful PCL film fabrication by spin coating a solution consisting of PCL, acetone and Dichloromethane (DCM) to achieve a film thickness from 40 to $105 \mu m^{89,90}$. In Luo et al.'s research, a PCL spacer is fabricated on a PLA layer structure by spray coating it with a thin layer (less than $1 \mu \mathrm{m}$ ) of PCL in a DCM solution ${ }^{91}$. All of these research works illustrate the possibility of making the PCL microchannels via a spin coating process.

If the exact size of a PCL microswimmer needs to be defined, the photolithography process can be utilized as the previous way to make a parylene swimmer. A feasible process could be (1) Use the photoresist to define the microchannel shape in a polydimethylsiloxane (PDMS) substrate followed by another PCL spin coating to make the side and top walls of the microswimmer. (2) Fabricate the bottom wall of the microswimmer by spin coating it to another flat PDMS substrate. (3) Adhere the top, side PCL walls to the PCL bottom of the microswimmer with the two PDMS substrates together by hot bonding them under the melting point of PCL ${ }^{92}$ (3) Separate the bottom of the PCL microstructures from the silicon substrate that will give a flat surface profile. (4) Use sputter to coat a thin aluminum layer and spin coat 
with photoresist. (5) Use photolithography to define the photoresist as a mask for dry etching of aluminum. (6) Use RIE process to dry etch aluminium to make the open end of PCL microchannels and cut them down from the surroundings. (7) Peel the microchannel structure off from the top PDMS substrate. The whole process is shown in Figure 3.1.

However, because of the complexity of this process, especially the difficulty of hot-bonding the top and bottom PCL layers due to the lack of proper equipment, we are simply using dipping method to fabricate the long-term biodegradable PCL device to demonstrate the idea: PCL is dissolved in an organic solution as the ingredient of $8 \mathrm{~g}$ of PCL, $40 \mathrm{~mL}$ of acetone, and $20 \mathrm{~mL}$ of DCM. The solution is heated by stirring hot plate (Corning ${ }^{\circledR}$, Model PC-420D, Corning Incorporated) at $75{ }^{\circ} \mathrm{C}$ which is about $10{ }^{\circ} \mathrm{C}$ above the melting point of PCL with a stirring speed of $100 \mathrm{rpm}$. This solution is heated and stirred for 3 hours to make sure PCL is totally dissolved and uniformly mixed. After the preparation of the PCL solution, a piece of optical fiber with a diameter of about $400 \mu \mathrm{m}$ is dipped into the solution quickly and taken out. As a result, the outside of the optical fiber is coated with a thin layer of the PCL solution. Notice that the PCL solution will dry very fast at room temperature, possibly due to the evaporation of acetone. After just several minutes, the coating solution will be solidified into PCL a layer on the outside of the optical fiber. Then, the PCL coating is held by a tweezer and the optical fiber is pulled out from the surrounding coating. In this way, a cylindrical PCL microchannel is fabricated. The PCL microchannel could be made into one end open or both ends open, depending on the PCL solution is coated to the tip of the optical fiber or in the middle. A sample of the channels has a diameter of $400 \mu \mathrm{m}$ and length of $1800 \mu \mathrm{m}$. This PCL microswimmer is used to test the propulsion ability. 


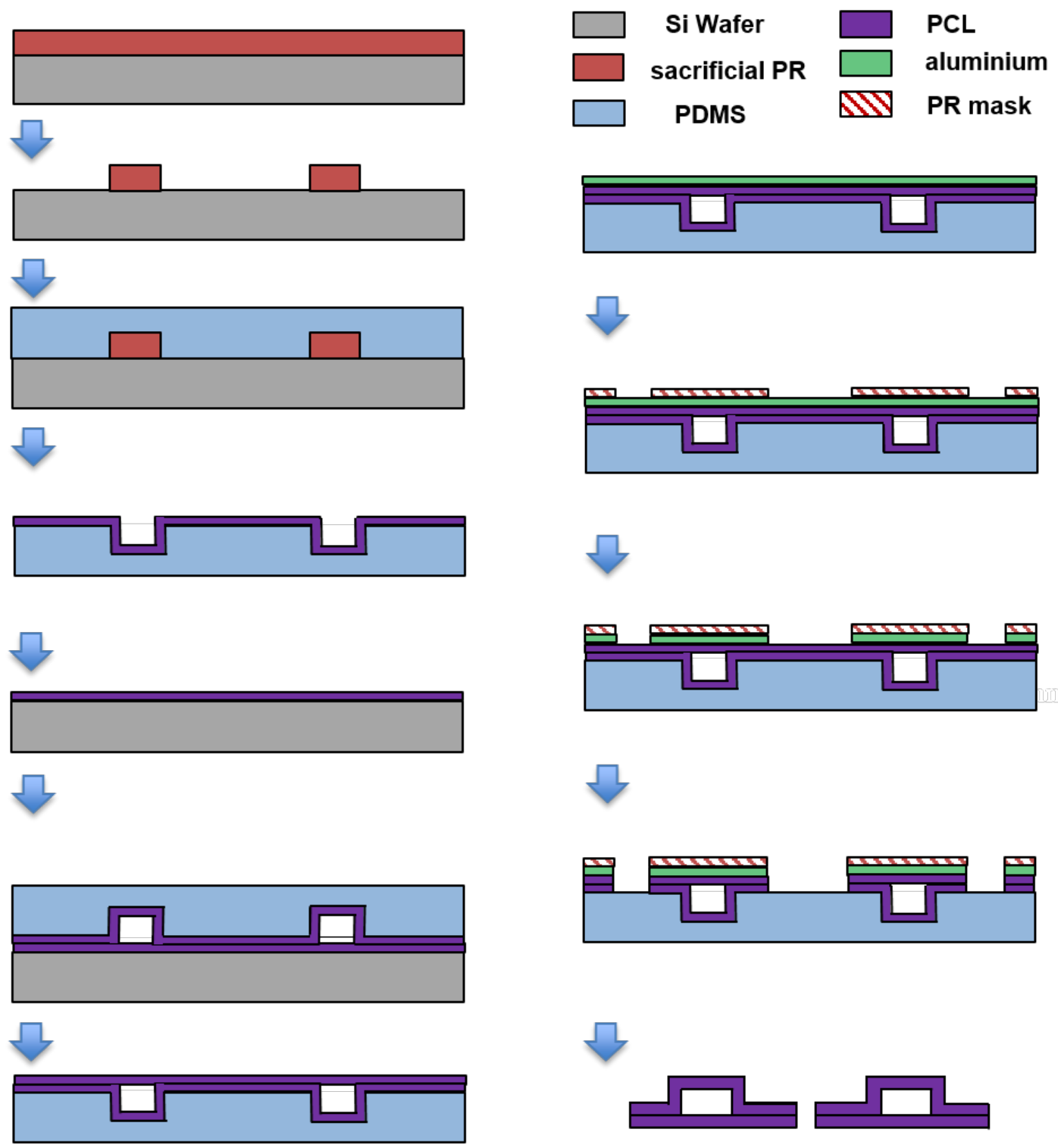

Figure 3.1. Fabrication process of one-end open PCL microchannel. 
However, because of the complexity of this process, especially the difficulty of hot-bonding the top and bottom PCL layers due to the lack of proper equipment, we are simply using dipping method to fabricate the long-term biodegradable PCL device to demonstrate the idea: PCL is dissolved in an organic solution as the ingredient of $8 \mathrm{~g}$ of PCL, $40 \mathrm{~mL}$ of acetone, and $20 \mathrm{~mL}$ of DCM. The solution is heated by stirring hot plate (Corning ${ }^{\circledR}$, Model PC-420D, Corning Incorporated) at $75{ }^{\circ} \mathrm{C}$ which is about $10{ }^{\circ} \mathrm{C}$ above the melting point of PCL with a stirring speed of $100 \mathrm{rpm}$. This solution is heated and stirred for 3 hours to make sure PCL is totally dissolved and uniformly mixed. After the preparation of the PCL solution, a piece of optical fiber with a diameter of about $400 \mu \mathrm{m}$ is dipped into the solution quickly and taken out. As a result, the outside of the optical fiber is coated with a thin layer of the PCL solution. Notice that the PCL solution will dry very fast at room temperature, possibly due to the evaporation of acetone. After just several minutes, the coating solution will be solidified into PCL a layer on the outside of the optical fiber. Then, the PCL coating is held by a tweezer and the optical fiber is pulled out from the surrounding coating. In this way, a cylindrical PCL microchannel is fabricated. The PCL microchannel could be made into one end open or both ends open, depending on the PCL solution is coated to the tip of the optical fiber or in the middle. A sample of the channels has a diameter of $400 \mu \mathrm{m}$ and length of $1800 \mu \mathrm{m}$. This PCL microswimmer is used to test the propulsion ability.

To carry out the experiment, the sample is put into a miniature cylindrical glass pipe (2 $\mathrm{mm}$ diameter) which is anchored at the bottom of the acrylic water tank. This water tank is exactly the same as used in previous sections 2.3 and 2.4. Then both the tank and the glass pipe are filled with deionized water and the water depth is kept at $3 \mathrm{~cm}$. When the PCL microchannel is submerged in water, the bubble is successfully trapped inside. However, in this PCL 
microchannel sample, both of the two ends' open are shape, therefore two menisci are formed in the microswimmer and when the acoustic wave is input, both of them could get in phase oscillation at the same time. An acoustic signal is generated by the piezoelectric actuator and applied to the testing water tank. The input acoustic frequency is swept, as stated in the previous experiments to find out the operation frequency that could generate larger bubble oscillation amplitude, and therefore test the underwater propulsion ability.

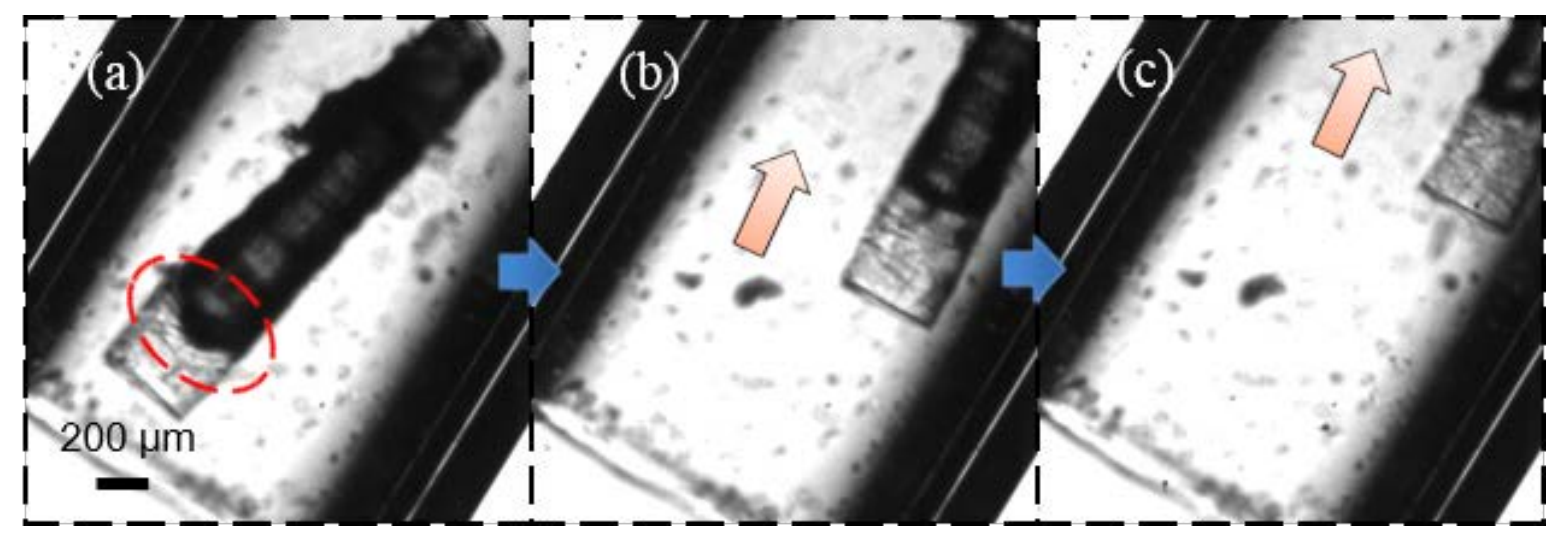

Figure 3.2. PCL microswimmer propulsion in the glass pipe.

This microswimmer gets a very fast propulsion speed which is $25.7 \mathrm{~mm} / \mathrm{s}$ with the input signal of $4.75 \mathrm{kHz}$ and $415 V_{p p}$ as shown in Figure 3.2. The microswimmer hit with the inner wall of the glass pipe while swimming, but the device is still propelled in the desired direction, which is shown by the red arrow. It should be noted that even though both of the two menisci are oscillating under the same acoustic field, and thus two microstreamings are generated at both ends of the microswimmer, the device only swims in one direction, which is consistent for testing several times. The main reason is that the oscillation amplitudes of the two menisci, thus the generated microstreamings, are quite different. The meniscus in the red dashed circle gets a 
much higher oscillation amplitude than the other one. This phenomenon could be attributed to the different diameters at the two ends of the microswimmers (red dashed circle end $400 \mu \mathrm{m}$; the other end $220 \mu \mathrm{m}$ ) and different surface roughness inside which will make different resistances to meniscus oscillation. As a result, the device is always pushed back by the meniscus that has stronger oscillation amplitude thus larger propulsion force.

On the other hand, this microswimmer is always propelled in the same direction even under different experiment conditions including $4.75 \mathrm{kHz} 415 V_{p p}, 3.40 \mathrm{kHz} 295 V_{p p}$ and $9.20 \mathrm{kHz} 235$ $V_{p p}$ (the last two propulsion results are not shown here). In other words, the meniscus in the red dashed circled consistently generates lager oscillation amplitude. This phenomenon is different from the previous bi-directional propulsion experiments in which two menisci of the two microbubbles have different oscillation amplitudes at their corresponding frequencies. The reason is that the microbubble trapped inside of the PCL microchannel is a whole body. If the acoustic frequency is close to the resonance, the whole microbubble is getting stronger expansion and shrinking. Therefore, both of the two menisci oscillation amplitude will be increased, contrary to when acoustic frequency is shifted far away from the resonance. The two menisci oscillation amplitudes will have the same increasing or decreasing trend when the acoustic frequency is changed. As a result, propulsion force generated by one end is always stronger than the other one and the propulsion direction is fixed.

According to the experiment, it could also be seen that the PCL walls are not as strong as parylene walls in previous experiments 2.3 and 2.4, as a result, they also became deformed with the oscillation of the microbubble. 


\subsubsection{Short-term Biodegradable Device}

For short-term application, metals are better because of their much faster in vivo degradation rate. Magnesium, with a density about $1.74 \mathrm{~g} / \mathrm{cm}^{3}$, is one of the exceptional lightweight biodegradable metals. Compared with other metals, magnesium does not have severe toxicity under the daily allowance and it is also essential for humans as shown in Table $5^{93-96}$. Magnesium has a corrosion rate of about $1 \mu \mathrm{m} /$ day in the simulated body fluid (SBF) ${ }^{97}$. Therefore, the corrosion rate is always too fast for both magnesium and magnesium alloy to be used as a bone implants or metal stents, and a lot of research is focused on improvement of corrosion resistance ${ }^{93,98}$. However, its fast degradation rate makes it an appropriate material to be used as a short-term drug delivery micro device. All of the above reasons make magnesium the first choice to be used in the device fabrication for short-term use.

In our experiment, the magnesium device is designed in a microtube shape with a wall thickness of hundreds of nanometers and a tube diameter of hundreds of microns. The fabrication process cannot be done by the previous fabrication method for parylene in section 2.3.2.1, therefore we will develop a new method based on the rollup process.

The rollup process is widely used to fabricate the micron/nano tubes. The traditional rollup method includes methods that are 'general' and 'specialized' procedure ${ }^{99}$. Both of them are based on the deposition process, selective etching of the sacrificial layer, and the elasticity of the tube material. Besides this common ground, the 'general' method does not have any external control of the rollup process and it is mainly decided by the thickness of the deposited layer. This process is basically a procedure of wrapping up and folding back. Therefore, it is easy to cause a large overlapped area and difficult to control the shape of the tube. On the other hand, the 'specialized' method based on inherently built-in strain is more precisely controlled and thus 
widely investigated ${ }^{100}$. For example, Deneke et al. showed the rollup process to fabricate the In(Ga)As/GaAs nanotubes by lattice mismatch induced built-in strain and investigated the effect of bilayer thickness and temperature ${ }^{101}$. Later, Songmuang et al. developed this technique to make pure Si and InGaAs nanotubes. It required a huge strain gradient from a specific sacrificial layer by lattice mismatch ${ }^{102}$. Then, the technique is further developed for different single material and material combinations on top of photoresist as the sacrificial layer. However, it needs an accurate control of environmental parameters such as deposition rate and temperature ${ }^{103}$. A lot of researchers use this technology to build the device for applications, such as the microfluidic tubes ${ }^{104}$, the optical resonator ${ }^{105}$, cell culture scaffolds ${ }^{106}$, catalytic jet engine $^{107}$, magnetic microdrillers ${ }^{108}$ and even complex structured metal/insulator micromesh ${ }^{109}$. However, it can be seen that this 'specialized' method requires complex temperature or deposition rate control setups or capability of multiple depositions of different materials. Therefore, in this paper, we developed a new simple method to make the microtube. Unlike the previous 'general' method, the size of the tube is not only decided by the material itself, such as the thickness of the film, but also controlled by the external applied mechanical strain. In contrast to the 'specialized' method, this process does not need a complex control of the deposition parameters, such as temperature or deposition rate. It does not need specific material combinations to generate the huge built-in strain either. We applied this method to different materials including magnesium, aluminium and parylene and showed successful rollups. Furthermore, we use the fabricated magnesium microtube to realize the underwater propulsion by acoustic bubble that is trapped inside of the microtube. The biodegradation of the microswimmer is also tested. The result shows the possibility of developing a biodegradable in vivo microswimmer to realize bio-sensing, drug delivery, and micro surgery in the future. 
Table 5. The summary of the pathophysiology and toxicology of Mg and some alloying elements

\begin{tabular}{|c|c|c|c|c|}
\hline Element & Name & $\begin{array}{l}\text { Blood Serum } \\
\text { Level }\end{array}$ & Toxicology & Daily Allowance \\
\hline \multirow{4}{*}{ Essential Element } & Mg & $0.9 \mathrm{mmol} / \mathrm{L}$ & $\begin{array}{l}\text { almost no evidence indicates } \\
\text { toxicity of magnesium }\end{array}$ & $0.7 \mathrm{~g}$ \\
\hline & $\mathrm{Ca}$ & $1.3 \mathrm{mmol} / \mathrm{L}$ & $\begin{array}{l}\text { calcium metabolism disorder; } \\
\text { kidney stones }\end{array}$ & $0.8 \mathrm{~g}$ \\
\hline & $\mathrm{Zn}$ & $46 \mu \mathrm{mol} / \mathrm{L}$ & $\begin{array}{l}\text { neurotoxic and hinder bone } \\
\text { development at higher } \\
\text { concentration }\end{array}$ & $15 \mathrm{mg}$ \\
\hline & Mn & $1 \mu \mathrm{mol} / \mathrm{L}$ & $\begin{array}{l}\text { excessive Mn results } \\
\text { in neurotoxic }\end{array}$ & $4 \mathrm{mg}$ \\
\hline $\begin{array}{c}\text { Potential Essential } \\
\text { Element }\end{array}$ & $\mathrm{Si}$ & - & $\begin{array}{l}\text { excessive } \mathrm{SiO} 2 \text { causes } \\
\text { lung diseases }\end{array}$ & - \\
\hline \multirow{4}{*}{ Other Element } & $\mathrm{Li}$ & $2-4 \mathrm{ng} / \mathrm{g}$ & $\begin{array}{l}\text { reduced kidney function and } \\
\text { central nervous system disorders }\end{array}$ & $0.2-0.6 \mathrm{mg}$ \\
\hline & $\mathrm{Al}$ & $2.1-4.8 \mu \mathrm{g}$ & $\begin{array}{l}\text { neurotoxic and accumulation } \\
\text { in bone }\end{array}$ & $\begin{array}{l}\text { total amount in } \\
\text { human }<300 \mathrm{mg}\end{array}$ \\
\hline & $\mathrm{Zr}$ & Total $<250 \mathrm{mg}$ & $\begin{array}{l}\text { high concentration in liver } \\
\text { and gall bladder }\end{array}$ & $3.5 \mathrm{mg}$ \\
\hline & Y\&RE & $<47 \mu g$ & $\begin{array}{l}\text { accumulation in bone } \\
\text { and liver }\end{array}$ & - \\
\hline
\end{tabular}




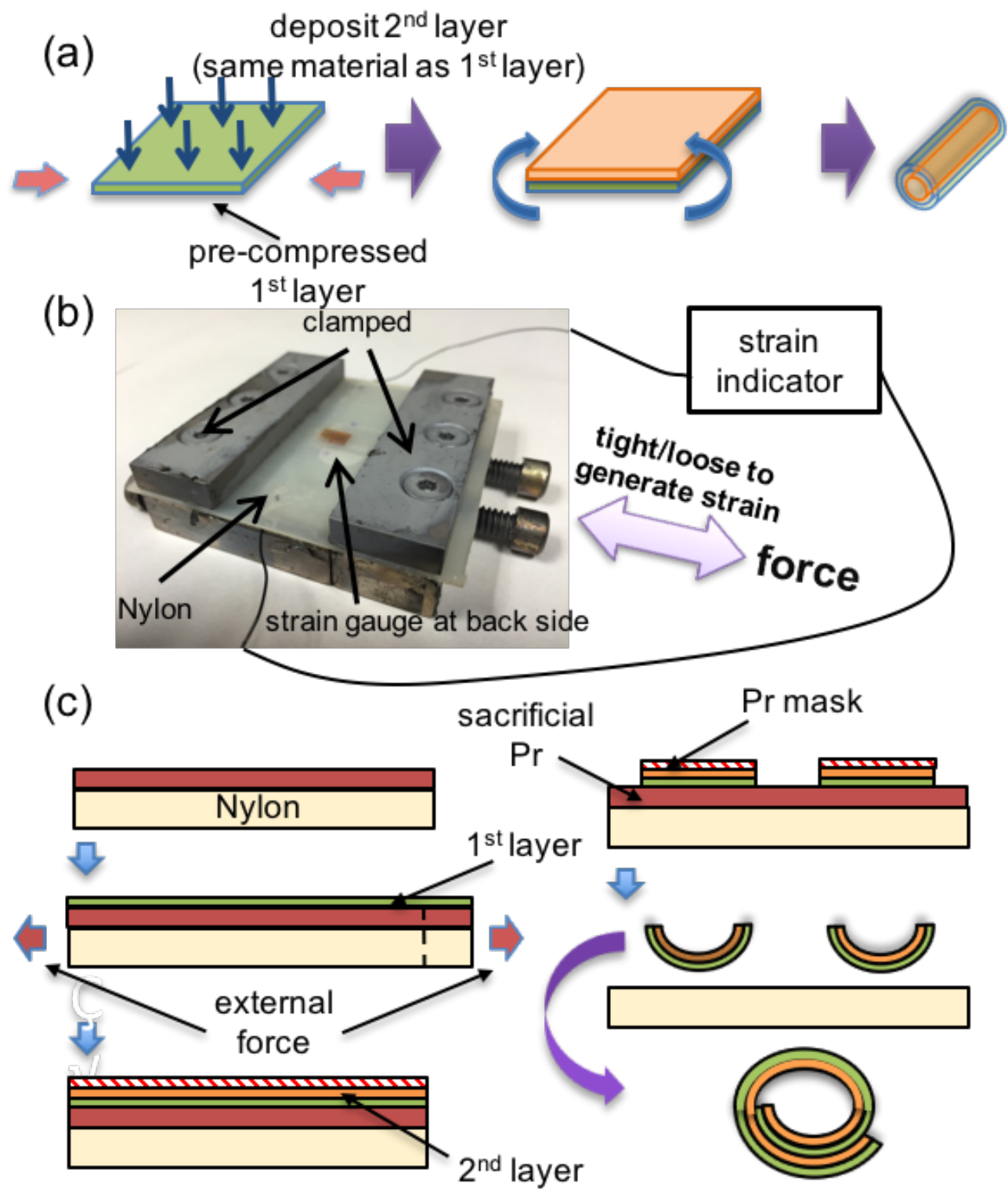

Figure 3.3. Microtube (a) fabrication concept, (b) strain generation between two layers by mechanical stretcher and (c) fabrication process flow. 
As mentioned in other researchers' work, the residual stress between different layers of the material could lead to a pure bending moment of a thin plate/film ${ }^{110,111}$ which will make the film roll up into a cylindrical shape. Previously, most of the stress between each layer of the material is caused by the deposition stress or different thermal contraction. However, in this paper, the stress mismatch will be built by the strain gradient in the direction of thickness by the mechanical method: the concept is shown in Figure 3.3 (a). The first layer is pre-compressed mechanically while the second layer is not, therefore the stress mismatch or strain gradient is formed.

In order to get the stress mismatch, a custom-made mechanical stretcher is used, as shown in Figure 3.3 (b). There are two stainless steel clamping parts that could tightly hold each edge of a flexible substrate. Grooves are made on the contact surfaces of the clamping parts that help generate a relatively uniform strain distribution. Otherwise the stress will be focused around the three screws that tighten the upper and bottom parts of the clamper therefore plastic deformation will be generated. Two driving screws will control the distance between the two clamping parts. When the driving screws are tightened, strain will be generated on the flexible substrate and recorded by a strain gauge attached to the back of the substrate. On the contrary, when the two screws are released, strain on the substrate will be reduced. In our experiment, Nylon6/6 is chosen as the flexible substrate. The reason is that its chemical stability is compatible with the photolithography process. Secondly, it has excellent stretch ability and large elastic deformation range.

The fabrication process is shown in Figure 3.3 (c). In the first step, a $1 \mu \mathrm{m}$ photoresist sacrificial layer (AZ ${ }^{\circledR} \mathrm{P} 4210$ Photoresist, AZ Electronic Materials) is spin coated on the polished Nylon 6/6 substrate with a spin speed of $5000 \mathrm{rpm}, 500 \mathrm{rpm}$ acceleration and total spin time of 
45 s (AZ ${ }^{\circledR}$ P 4000 Series Spin Speed Curve, AZ Electronic Materials, AZ ${ }^{\circledR} \mathrm{P} 4210$ Photoresist, AZ Electronic Materials). $90{ }^{\circ} \mathrm{C}$ soft baking is done in an oven for about $20 \mathrm{~min}$ instead of the 30 min provided by the data package. The reason is that it was found that longer time (30 $\mathrm{min}$ ) makes the photoresist layer easy to generate cracks when strain is applied, possibly because the solvent is totally evaporated. At the same time, it was found that $20 \mathrm{~min}$ is already long enough to solidify the photoresist. Then, the Nylon substrate is loaded onto the stretcher. Two driving screws in the stretchers are tightened which could generate the tension depending on what is needed. In this experiment, the tension is generated up to $5000 \mu \varepsilon$. Greater than this will break the photoresist sacrificial layer or cause plastic deformation on the Nylon 6/6 substrate. Then the sample, together with stretcher, is coated with the first layer of the microtube material by either the sputter (Innovative, System Engineering, INC.) for 160/220 nm magnesium (Purity 99.9\%, Angstrom Sciences, INC.), or $160 \mathrm{~nm}$ aluminium (Purity 99.999\%, Angstrom Sciences, INC.), or the CVD process (LABCOATER ${ }^{\circledR} 2$ Parylene Deposition Unit, Model PDS 2010, Special Coating System, INC.) for $160 \mathrm{~nm}$ parylene. Then the stain of the substrate coated with the first is removed by releasing the two driving screws. Notice that the strain change during this release is also recorded by the strain gauge. This release strain is always not the same as previous stretch strains. A possible reason is that there is still some plastic deformation on the Nylon substrate caused by uneven stress distribution although the grooves are added to the stretcher. Another reason is that the sputtered aluminum layer would have an effect on the Nylon substrate that stops the Nylon from going back to the exact original shape. After releasing the strain, the sample with stretcher is coated with the second layer of the microtube material by using exactly the same method and getting the same thickness. Through this process, the stress mismatch between the first and second material layers is generated and maintained. The first layer tries to 
extend and elongate to the original shape while the second layer tries to retract. As a result, when the two-layers structure is released from the substrate, it will roll up. After the second microtube material layer is sputtered, the Nylon substrate is unloaded from the stretcher and the second photoresist layer is spin coated with a thickness of $2 \mu \mathrm{m}$, at a spin speed of $3000 \mathrm{rpm}$ for $45 \mathrm{~s}$ (AZ ${ }^{\circledR}$ P 4000 Series Spin Speed Curve, AZ Electronic Materials, AZ ${ }^{\circledR}$ P 4210 Photoresist, AZ Electronic Materials). This layer is used as a mask to define the shape of two-layer microtube material during the following etching process. After exposure, the mask photoresist layer is patterned by the photoresist developer $\left(\mathrm{AZ}{ }^{\circledR} \mathrm{P}\right.$ 400K Developer Diluted 1:3, AZ Electronic Materials). Depending on the microtube material, either wet etching $\left(\mathrm{AZ}^{\circledR} \mathrm{P}\right.$ 400K Developer Diluted 1:4, AZ Electronic Materials for aluminium, $\mathrm{FeCl}_{3}$ copper etchant solution, $\mathrm{MG}$ Chemicals for magnesium) or dry etching (reactive ion etching by Phantom Ш LT, Trion Technology for parylene) is used to pattern the shape of the material. In our experiments, all of the microtube materials are patterned into circles $2 \mathrm{~mm}$ in diameter. Finally, the sample is put into an acetone solution, and the acetone could remove both the sacrificial photoresist layer and the mask photoresist layer. Consequently, the two-layer material structure is rolled up due to the mechanically built-in stress. Therefore, the microtubes are fabricated with a wall thickness of several hundreds of nanometers, a diameter of several hundreds of microns, and a length about 2 $\mathrm{mm}$.

\subsubsection{FABRICATION RESULTS}

Figure 3.4 (a) shows the pattern of magnesium just before acetone etching which is a circle with a diameter of $2 \mathrm{~mm}$. When it is put into acetone, the roll up happens. Notice that the rollup axes are almost perpendicular to stretching direction. As shown in Figure 3.4 (b), the pre-strain direction is in $x$ direction therefore the tube rolls up the same direction and the microtube axe is 
in $y$ direction. Control experiments were also carried out without this pre-strain process. The result shows that the circle pattern will either stay flat without rollup or curve up a little bit but in a random direction as shown in Figure 3.4 (c). It proves that the rollup phenomenon is caused by the mechanical stress mismatch between two layers of the material instead of the 'general' rollup method as mentioned previously. 

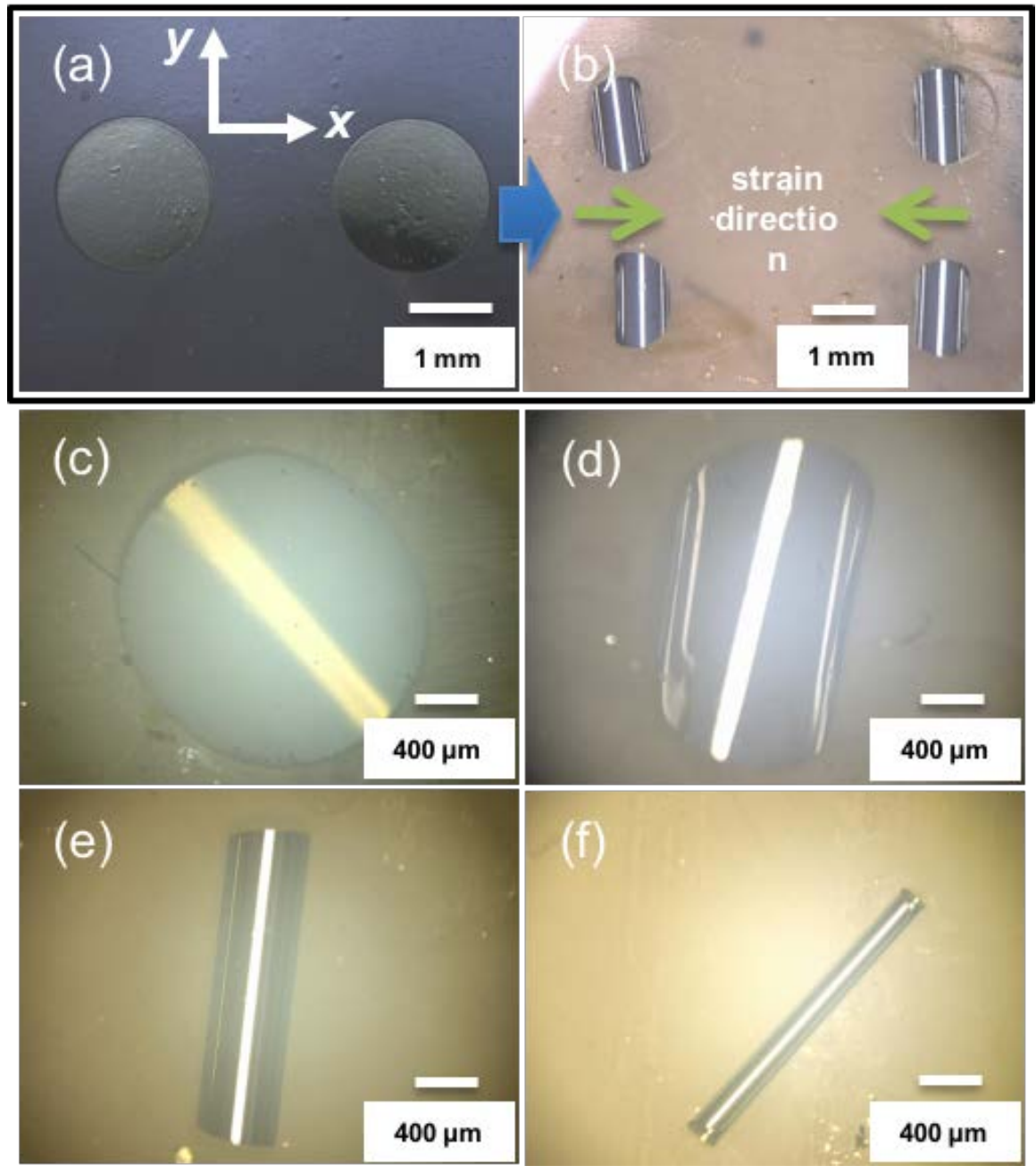

Figure 3.4. Magnesium film rollup (a) before acetone etching of sacrificial Pr, (b) during acetone etching, (c) without pre-strain in the $1^{\text {st }}$ layer, (d) $1200 \mu \varepsilon$ pre-strain in the $1^{\text {st }}$ layer, (e) $2000 \mu \varepsilon$ pre-strain in the $1^{\text {st }}$ layer and (f) $3800 \mu \varepsilon$ pre-strain in the $1^{\text {st }}$ layer. 
According to the theory for the pure bending film ${ }^{111}$, strain gradient is one of the most important parameters to decide the diameter of the microtube. Therefore, the different pre-strains are tested on the magnesium film. Notice that due to the plastic deformation generated during first material layer stretching process, the strain in stretching is always larger than the one during releasing. What is more, the strain during releasing actually determines the stress mismatch between the first and second layers. Therefore, the strain during releasing is recorded as the strain parameter. Figure 3.4 (d) to (e) shows the radius of the microtube is getting smaller with the increase of applied pre-strain. The same method is also fabricated for the aluminium (two layers structure, $130 \mathrm{~nm}$ for each layer) and parylene (two layers structure, $160 \mathrm{~nm}$ for each layer) tube and the results are shown in Figure 3.5. The rollup behaviors are pretty similar with the magnesium tube. For the aluminium tube, $1100 \mu \varepsilon$ pre-strain could make the microtube radius larger. When the pre-strain is increased to $1900 \mu \varepsilon$, the tube diameter will get smaller. In all the cases, the axes of microtubes are perpendicular to the strain direction. The same phenomenon happens for parylene film rollups with pre-strain of $1800 \mu \varepsilon$ and $3200 \mu \varepsilon$.

Notice that if the pre-strain is too large, for example $4000 \mu \varepsilon$ for the $130 \mathrm{~nm}$ aluminium layer in our test, the first stretched layer and the sacrificial photoresist layer will be easily detached during the pre-strain releasing process. As a result, the aluminium layer will pop up in a straight line and form a bumper shape as shown in Figure 3.6. The height of the pop up is measured at about $6 \mu \mathrm{m}$. All of the pops up are in the same direction that is perpendicular to the pre-strain direction and in the similar size. It also confirms that the rollups are caused by the mechanical stretching process instead of the mechanism mentioned in others' work ${ }^{99}$. 


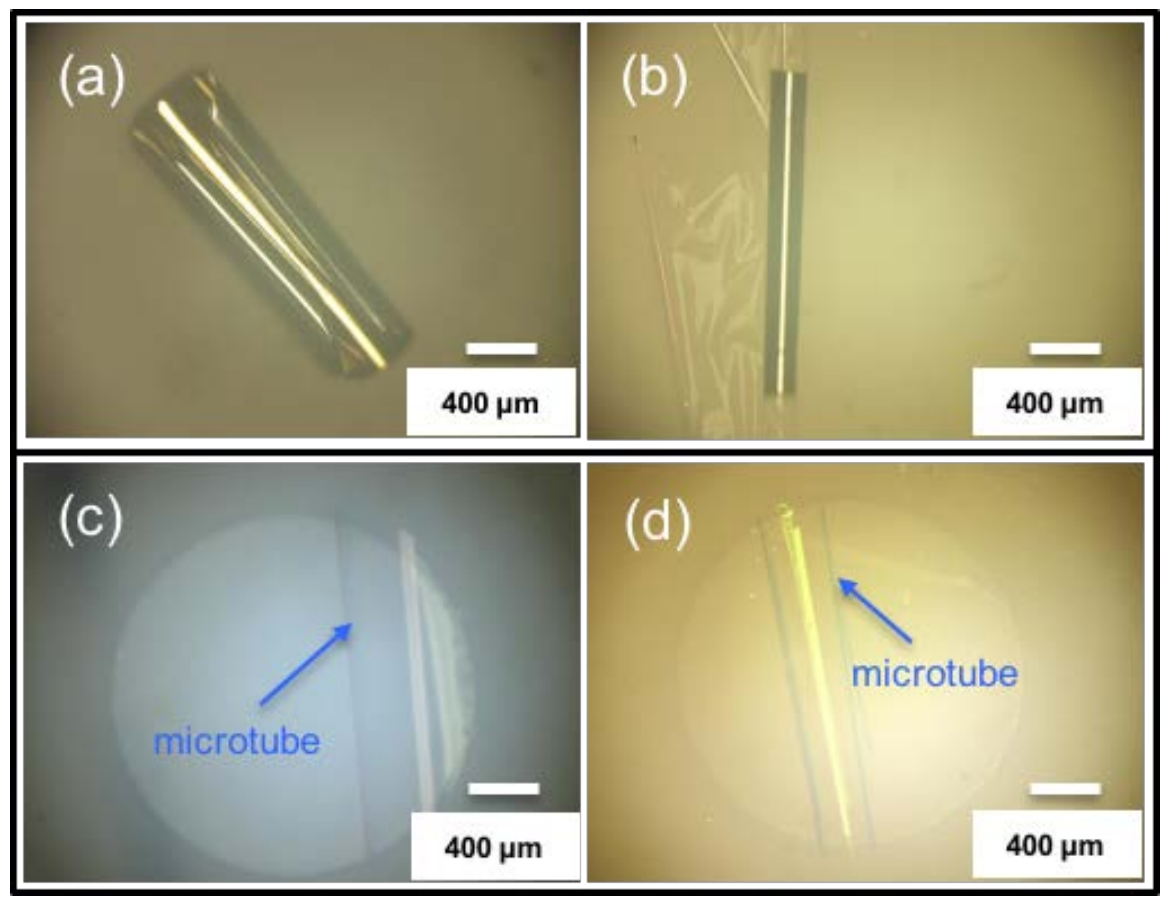

Figure 3.5. Aluminium film rollup (a) $1100 \mu \varepsilon$ pre-strain and (b) $1900 \mu \varepsilon$ pre-strain in the 1 st layer. Parylene film rollup (c) $1800 \mu \varepsilon$ pre-strain and (d) $3200 \mu \varepsilon$ pre-strain in the 1st layer.

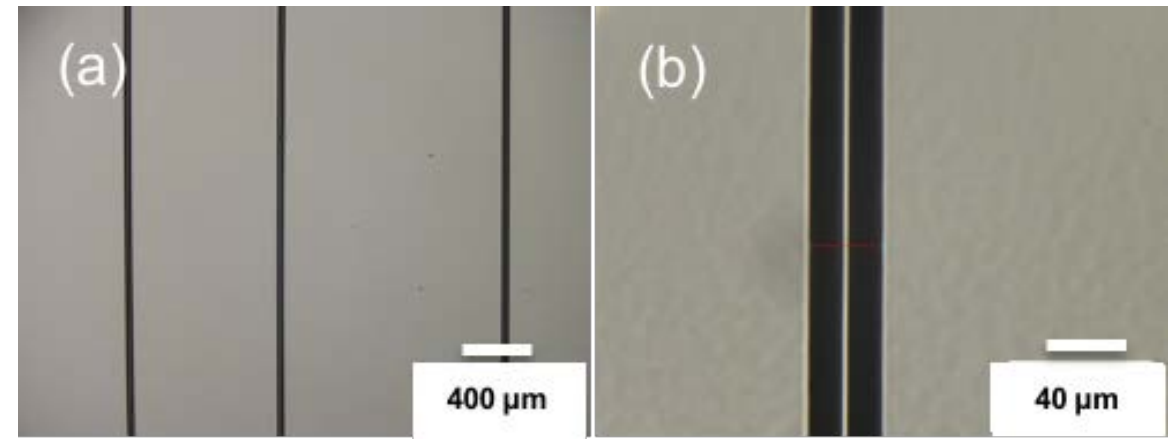

Figure 3.6. $130 \mathrm{~nm}$ thick aluminium layer detached at $4000 \mu \varepsilon$ (a) overall view of the pops up straight lines pattern and (b) an enlarged view. 
In order to find the accurate relation between magnesium radius and the pre-strain, the images of twenty different microtubes made in the same fabrication process are captured for each strain condition. Then the radius is calculated in the following method as shown in Figure 3.7. For those which could roll up for more than one lap (always for larger pre-strain samples), the radius is directly measured through the captured image, while for those that cannot roll up for a intact tube (always for smaller pre-strain samples) the radius is calculated from the captured image by the curvature, then all the radii are averaged. Note that due to the uneven distribution of the prestrain which might be caused at different position on the same substrate, the microtube radius may vary as shown by the error bar. The result shows that when the pre-strain is $1200 \mu \varepsilon$, the average radius of the tube is about $598 \mu \mathrm{m}$ while when the pre-strain is increased to $2000 \mu \varepsilon$, the radius is reduced to $269 \mu \mathrm{m}$. At the same time, as the pre-strain increases, the radius variation of the twenty microtubes is also reduced. In other words, larger pre-strain could make rollup results more consistent.

According to the theoretical formula ${ }^{111}$, the radius of the microtube could be expressed as

$$
R_{0}=\frac{t}{(1+v) \Delta \varepsilon}
$$

where $R_{0}$ is the radius of the microtube, $t$ is the thickness of the film, $v$ is the Poisson's ratio and $\Delta \varepsilon$ is the strain gradient of the film. However, in our experiment, the recorded pre-strain may not be exactly the strain gradient of the film, therefore we built a linear expression between the prestrain reading and the strain gradient of the film, which is

$$
\Delta \varepsilon=a \cdot \varepsilon+b
$$

where $a$ and $b$ are the curve-fitting coefficient. Put Equation (13) into Equation (12),

$$
R_{0}=\frac{t}{(1+v)(a \cdot \varepsilon+b)}
$$


use Equation (14) and the previous experimental result, coefficient $a$ and $b$ can be calculated. As a result, the radius of the magnesium microtube is expressed as,

$$
R_{0}=\frac{t}{(1+v)(a \cdot \varepsilon+b)}=\frac{t}{(1+v)\left(0.875 \varepsilon-0.48 \times 10^{-3}\right)}
$$

To further reduce the radius of the magnesium microtube, based on the Equation (15), the thickness of sputtered magnesium film is decreased to $160 \mathrm{~nm}$ and the pre-strain is increased to $3800 \mu \varepsilon$ as shown in Figure 3.4 (f). The experiment result shows that the radius of microtube is $88 \pm 7 \mu m$ which is quite close to the calculation prediction based on Equation (15), $87 \mu m$. This kind of magnesium tube is tested for underwater propulsion later in this section.

The relations between pre-strain and microtube diameters for all other materials are also calculated. For aluminium film, $1100 \mu \varepsilon$ will make the microtube average radius about $309 \mu m$ and $1900 \mu \varepsilon$ will make the average radius about $169 \mu \varepsilon$. For parylene film, $1800 \mu \varepsilon$ will make the average radius about $380 \mu m$ and $3200 \mu \varepsilon$ will make the average radius about $171 \mu \varepsilon$. Therefore, the curve fitting for aluminium and parylene are expressed respectively, as

$$
\begin{aligned}
& R_{0}=\frac{t}{(1+v)(a \cdot \varepsilon+b)}=\frac{t}{(1+v)\left(0.65 \varepsilon-0.095 \times 10^{-3}\right)} \\
& R_{0}=\frac{t}{(1+v)(a \cdot \varepsilon+b)}=\frac{t}{(1+v)\left(0.54 \varepsilon-0.372 \times 10^{-3}\right)}
\end{aligned}
$$

All of the curve fittings are shown in Figure 3.7, 


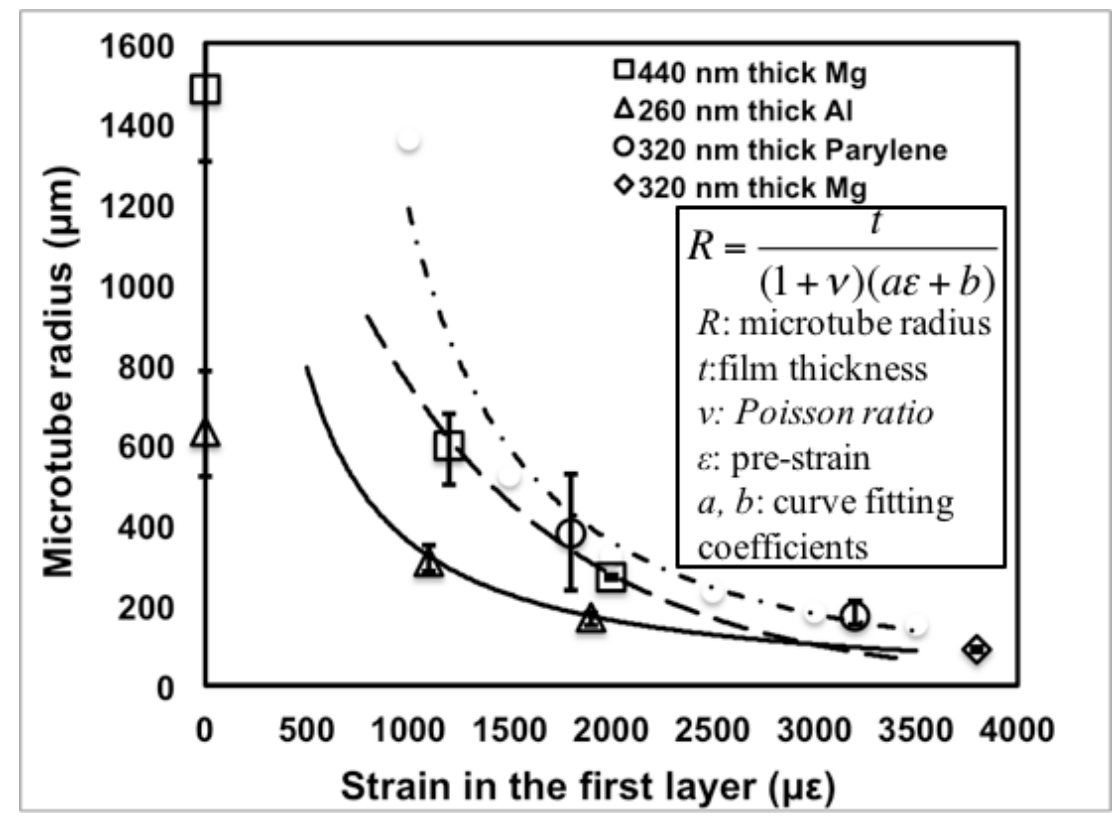

Figure 3.7. Microtube radius vs. pre-strain for magnesium, aluminium and parylene film with curve-fits by Eq. (14).

\subsubsection{ACOUSTIC BUBBLE PROPULSION AND BIODEGRADATION RESULT}

The aluminium and magnesium microtubes are strong enough to hold the cylindrical shape without collapse when they are naturally dried in air as shown in Figure 3.8 (a). When the aluminium microtubes are submerged in water, air bubbles will be trapped inside the tube automatically. The water level is kept at $3 \mathrm{~cm}$ in an acrylic water tank $11 \times 11 \times 5 \mathrm{~cm}^{3}$. Then, the acoustic wave is generated by a piezoelectric actuator attached to the side wall of the water tank. When the acoustic wave is generated at about $11 \mathrm{kHz}$, the trapped microbubble is shrinking and expanding periodically and microstreaming is generated. Soda lime glass microspheres (Duke Scientific Corporation) with $2 \mu \mathrm{m}$ in diameter are seeded into water to show the microstreaming phenomenon. As seen from Figure 3.8 (b), even though both ends of the aluminium microtubes 
were open, the bubble generated streaming at both ends therefore the microparticles are pushing away from two ends and two empty regions are formed which makes propulsion possible.

In order to demonstrate the ability of underwater propulsion, microstreaming generated at only one end is preferred. Otherwise two forces at each open end of the microtube will be in opposite directions and cancelled out. Therefore, one of the magnesium microtubes is picked up and one end is sealed by glue. $1 \%$ Teflon solution (Dupont Polymers) is coated inside of the tube by capillary force to make the inside magnesium surface hydrophobic, therefore bubbles will be easily trapped inside the magnesium tube when submerged in water. This microtube is put into water and an acoustic wave is applied. The result shows that the propulsion force is generated at only one end at $11 \mathrm{kHz}$ which is close to the resonance of the acoustic system, and the bubble oscillation amplitude is magnified therefore the force is strong enough to push the microtube as shown in Figure 3.8 (c) and (d). The time internal between these two figures are about $20 \mathrm{~ms}$. 


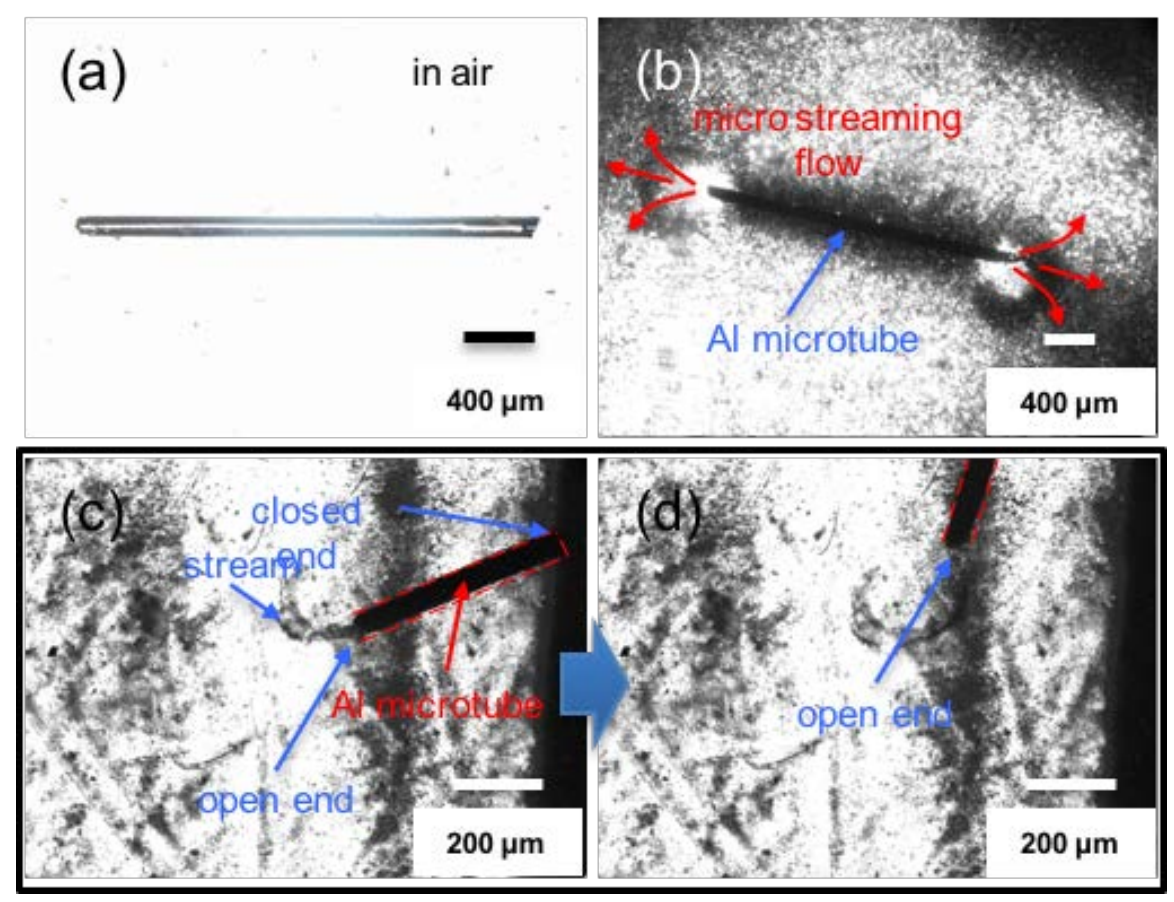

Figure 3.8. (a) Aluminium microtube dried in air, (b) trapped bubble oscillation in water (particle seeded for flow visualization) and magnesium microtube (c) start of propelling motion, (d) end of propelling motion by acoustic bubble oscillation $\left(11.0 \mathrm{kHz}, 175 V_{p p}\right)$.

The biodegradability is also tested for the magnesium microtube. Unlike other materials, such as zinc or calcium, magnesium is not only a daily essential element, but it has almost no evidence of toxicity. What is more, its daily allowance is about $0.7 \mathrm{~g}$, which is much higher than the weight of our microswimmer (about $1.75 \mathrm{mg}$ ). Therefore, we tested the magnesium microtube biodegradability in tap water to confirm its potential to be used for in vivo swimmer. The result is shown from Figure 3.9 (a) to (b). When the magnesium microtube is just put into tap water, the structure is intact. However, after about one day, most of the magnesium is dissolved and only a transparent layer is left. The transparent layer is speculated to be magnesium oxide spontaneously formed during deposition that is a reaction product of magnesium and photoresist, and it needs more study. 


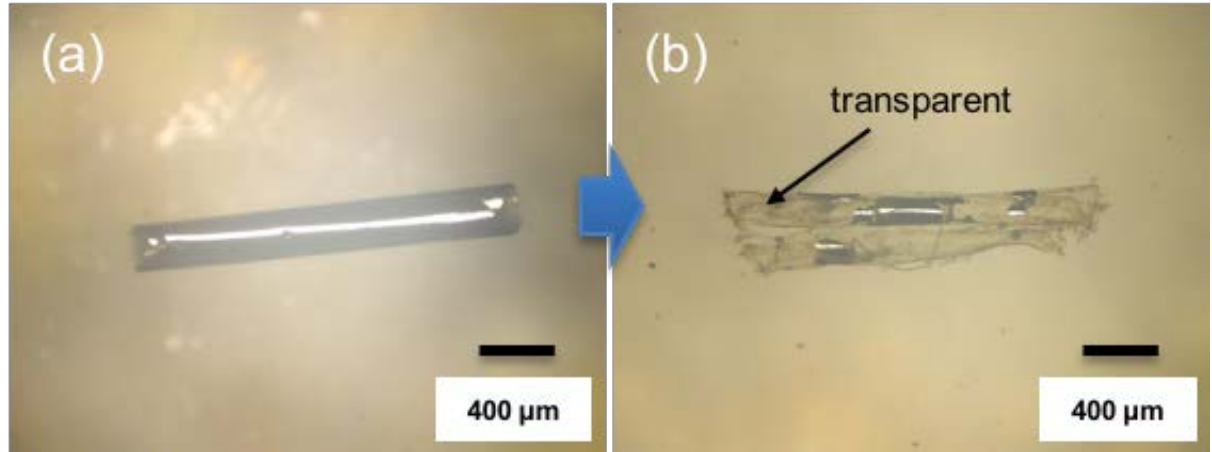

Figure 3.9. Magnesium microtube degraded in tap water at (a) initial time and (b) after 1 day.

In this section, we developed a new method to roll up the material with a thickness of hundreds of nanometers in range, therefore fabricate the microtubes. The key idea is to build stress difference between two layers of the material by a mechanical stretcher. The first layer of the material is pre-stretched while the second layer is not. As a result, when the two layers are combined and removed from the substrate, they will roll up to form a microtube (curvature). This method works for different kinds of materials. We presented the fabrication results for magnesium, aluminium and parylene. The method does not require a specific combination of different materials to generate the huge built-in stress. At the same time, it does not require a complex control of the material deposition rate or the deposition temperature. The microtube diameter is related to the built-in strain and the thickness of the material. As the pre-strain of the first layer is increased and the layer thickness is decreased, the diameter of the microtube could be decreased. The fabricated magnesium and aluminium microtubes are strong enough and keep a cylindrical shape after dried in air. When the two-ends-open microtubes are put into water, bubbles could be trapped inside of the tube and the microtube could overcome surface tension without collapsing. When an acoustic field is applied to this micro device, the magnesium and 
aluminium microtube wall is strong enough to hold the bubble oscillation inside. Microstreamings could be generated as long as the bubble oscillation is strong enough which always happens around the resonance of the acoustic system. Fluid visualization by seeded microsphere particles shows that the microstreaming happens at both of the two ends. In order to propel the micro device, one end of the magnesium microtube is sealed and successful propulsion behavior is demonstrated. At last, the biodegradability of the magnesium microtube is tested. After about one day, most of the magnesium will be dissolved in tap water. The experiments show the prospect of this type of device used in vivo to complete multiple missions such as drug delivery or navigation in the future. 


\subsection{CONCLUSION REMARKS}

This thesis reports a new type of micron size swimmer that is propelled by the microstreaming generated by bubble oscillation in an acoustic field. The operation concept is proven by computational fluid dynamics (CFD) simulation. Then, device propulsion ability and fluid visualization is demonstrated for one- and two-dimensional motion. At last the biodegradable device is also fabricated.

The simulation results from ANSYS Fluent ${ }^{\circledR}$ show that when the fluid discharges and intakes from a one-end open channel, the fluid patterns are different especially at large Reynolds $(R e)$ numbers, therefore, this difference can generate a propulsion force and push the microswimmer back in theory. What is more, bubbles can be oscillated in an acoustic field generated by a piezoelectric actuator with a large amplitude at the acoustic frequency close to the resonance of the acoustic system. As a result, discharge and intake of fluid could be formed periodically. Based on this concept, microswimmer are fabricated by photolithography from parylene structures. The microswimmer is a one-end open microchannel with the equivalent hydraulic diameter of $80 \mu \mathrm{m}$. Even though the size of the device is small, the operation frequency for it is always higher than several $\mathrm{kHz}$, which makes the Reynolds number large enough to generate propulsion force. The phenomenon is confirmed by fluid visualization. When the input voltage to the piezoelectric actuator is high enough at the resonance, the microswimmer could be propelled. One-dimensional linear motion, rotation and payload-carrying ability are demonstrated. 
Because this propulsion force is sensitive to the resonance frequency, different sizes of bubbles are fabricated by trapping into different size of microchannels. As a result, by switching acoustic frequencies, the short and long bubbles could be activated selectively. Consequently, by arranging short and long bubbles into different directions on the same microswimmer, the propulsion motion is further developed into bi-directional motion and two-dimensional steering motion.

In order to make the microswimmer available to complete in vivo missions, such as drug delivery or navigation through blood pipes, biodegradable materials are used. The long-term biodegradable microswimmer is made from PCL by dipping method and propulsion is demonstrated. On the other hand, magnesium is used to fabricate the short-term microswimmer. The key idea is to build stress mismatch between two layers of the same material by a mechanical stretcher. Because of this stress mismatch, the materials, including magnesium, aluminium, or parylene can roll up to form a microtube automatically. The underwater propulsion ability and biodegradable ability of magnesium tubes are demonstrated at last. 


\section{BIBLIOGRAPHY}

1. L. Rubinstein, A Practical Nanorobot for Treatment of Various Medical Problems, presented in part at the Proceedings of the 8th Foresight Conference on Molecular Nanotechnology, Bethesda, MD, USA, 2-5 November 2000.

2. G. Vince and C. Wilson, The rise of the miniature medical robots, New Scientist, 2009, 204, 50-53.

3. M. Sitti, Voyage of the microbots, Nature, 2009, 458, 1121-1122.

4. T. Fukuda, A. Kawamoto and F. M. Arai, H. , Mechanism and Swimming Experiment of Micro Mobile Robot in Water, presented in part at the Proceedings of the 1994 IEEE International Conference on Micro Electro Mechanical Systems, Oiso, Japan, 25-28 January 1994.

5. Z. Wang, Y. Wang, J. Li and G. Hang, A Micro Biomimetic Manta Ray Robot Fish Actuated by SMA, presented in part at the Proceedings of the 2009 IEEE International Conference on Robotics and Biomimetics, Guilin, China, 19-23 December 2009.

6. Z. Wang, G. Hang, J. Li, Y. Wang and K. Xiao, A micro-robot fish with embedded SMA wire actuated flexible biomimetic fin, Sens. Actuators A, 2008, 144, 354-360.

7. L. Shi, S. Guo and K. Asaka, A Novel Jellyfish-Like Biomimetic Microrobot, presented in part at the Proceedings of the 2010 IEEEI/ICME International Conference on Complex Medical Engineering, Gold Coast, Australia, 13-15 July 2010.

8. S. Guo, T. Fukuda and K. Asaka, A new type of fish-like underwater microrobot, IEEE/ASME Trans. Mechatron., 2003, 8, 136-141.

9. S. Guo and Y. Ge, Underwater Swimming Micro Robot Using IPMC Actuator, presented in part at the Proceedings of the 2006 IEEE International Conference on Mechatronics and Automation, Luoyang, China, 25-28 June 2006;.

10. N. Kamamichi, M. Yamakita, K. Asaka and Z.-W. Luo, A Snake-Like Swimming Robot Using IPMC Actuator/Sensor, presented in part at the Proceedings of the 2006 IEEE International Conference on Robotics and Automation, Orlando, FL, USA, 15-19 May 2006. 
11. B. Kim, D.-H. Kim, J. Jung and J.-O. Park, A biomimetic undulatory tadpole robot using ionic polymer-metal composite actuators, Smart Mater. Struct., 2005, 14, 1579-1585.

12. A. Ming, S. Park, Y. Nagata and M. Shimojo, Development of Underwater Robots Using Piezoelectric Fiber Composite, presented in part at the Proceedings of the 2009 IEEE International Conference on Robotics and Automation, Kobe, Japan, 12-17 May, 2009.

13. X. Deng and S. Avadhanula, Biomimetic Micro Underwater Vehicle with Oscillating Fin Propulsion: System Design and Force Measurement, presented in part at the Proceedings of the 2005 IEEE International Conference on Robotics and Automation, Barcelona, Spain, 18-22 April 2005.

14. J. Wang and K. M. Manesh, Motion control at the nanoscale, Small, 2009, 6, 338-345.

15. E. Lauga and T. R. Powers, The hydrodynamics of swimming microorganisms, Rep. Prog. Phys., 2009, 72, 096601.

16. C. Brennen and H. Winet, Fluid mechanics of propulsion by cilia and flagella, Ann. Rev. Fluid Mech., 1977, 9, 339-398.

17. R. J. Dijkink, v. d. D. Dennen, J.P., C. D. Ohl and A. Prosperetti, The "acoustic scallop": A bubble-powered actuator, J. Micromech. Microeng., 2006, 16, 1653-1659.

18. J. Feng and S. K. Cho, Micro Propulsion in Liquid by Oscillating Bubbles, presented in part at the Proceedings of the 2013 IEEE 26th International Conference on Micro Electro Mechanical Systems, Taipei, Taiwan, 20-24 January 2013.

19. L. Zhang, J. J. Abbott, L. Dong, K. E. Peyer, B. E. Kratochvil, H. Zhang, C. Bergeles and B. J. Nelson, Characterizing the Swimming Properties of Artificial Bacterial Flagella, Nano Lett., 2009, 9, 3663-3667.

20. A. Ghosh and P. Fischer, Controlled propulsion of artificial magnetic nanostructured propellers, Nano Lett., 2009, 9, 2243-2245.

21. P. Tierno, R. Golestanian, I. Pagonabarraga and F. Sagués, Magnetically Actuated Colloidal Microswimmers, J. Phys. Chem. B, 2008, 112, 16525-16528.

22. S. Martel, C. C. Tremblay, S. Ngakeng and G. Langlois, Controlled manipulation and actuation of micro-objects with magnetotactic bacteria, Appl. Phys. Lett., 2006, 89, 233904.

23. B. Behkam and M. Sitti, Bacterial flagella-based propulsion and on/off motion control of microscale objects, Appl. Phys. Lett., 2007, 90, 023902.

24. B. J. Williams, S. V. Anand, J. Rajagopalan and M. T. A. Saif, A self-propelled biohybrid swimmer at low Reynolds number, Nat. Commun., 2014, 5, 3081. 
25. J. R. Howse, R. A. L. Jones, A. J. Ryan, T. Gough, R. Vafabakhsh and R. Golestanian, Self-motile colloidal particles: From directed propulsion to random walk, Phys. Rev. Lett., 2007, 99, 048102.

26. J. G. Gibbs and Y.-P. Zhao, Autonomously motile catalytic nanomotors by bubble propulsion, Appl. Phys. Lett., 2009, 94, 163104.

27. J. Vicario, R. Eelkema, W. R. Browne, A. Meetsma, R. M. L. Crois and B. L. Feringa, Catalytic molecular motors: fuelling autonomous movement by a surface bound synthetic manganese catalase, Chem. Commun., 2005, 3936-3938.

28. A. A. Solovev, Y. Mei, E. B. Ureña, G. Huang and O. G. Schmidt, Catalytic microtubular jet engines self-propelled by accumulated gas bubbles, Small, 2009, 5, 1688-1692.

29. W. Gao, A. Uygun and J. Wang, Hydrogen-bubble-propelled zinc-based microrockets in strongly acidic media, J. Am. Chem. Soc., 2012, 134, 897-900.

30. T. R. Kline, W. F. Paxton, T. E. Mallouk and A. Sen, Catalytic Nanomotors: RemoteControlled Autonomous Movement of Striped Metallic Nanorods, Angew. Chem. Int. Ed., 2005, 44, 744-746.

31. S. Sundararajan, P. E. Lammert, A. W. Zudans, V. H. Crespi and A. Sen, Catalytic Motors for Transport of Colloidal Cargo, Nano Lett., 2008, 8, 1272-1276.

32. J. Burdick, R. Laocharoensuk, P. M. Wheat, J. D. Posner and J. Wang, Synthetic Nanomotors in Microchannel Networks: Directional Microchip Motion and Controlled Manipulation of Cargo, J. Am. Chem. Soc., 2008, 130, 8164-8165.

33. I. Buttinoni, G. Volpe, F. Kümmel, G. Volpe and C. Bechinger, Active Brownian motion tunable by light, J. Phys.: Condens. Matter, 2012, 24, 284129.

34. E. M. Purcell, Life at low Reynolds number, Am. J. Phys., 1977, 45, 3-11.

35. J. J. Abbott, K. E. Peyer, M. C. Lagomarsino, L. Zhang, L. Dong, I. K. Kaliakatsos and B. J. Nelson, How Should Microrobots Swim?, The International Journal of Robotics Research, 2009, 28, 1434-1447.

36. L. E. Becker, S. A. Koehler and H. A. Stone, On self-propulsion of micro-machines at low Reynolds number: Purcell's three-link swimmer, J. Fluid Mech., 2003, 490, 15-35.

37. A. Najafi and R. Golestanian, Simple swimmer at low Reynolds number: Three linked spheres, Phys. Rev. E, 2004, 69, 062901.

38. M. Leoni, J. Kotar, B. Bassetti, P. Cicuta and M. C. Lagomarsino, A basic swimmer at low Reynolds number, Soft Matter, 2009, 5, 472-476. 
39. M. S. Plesset and A. Prosperetti, Bubble Dynamics and Cavitation, Ann. Rev. Fluid Mech., 1977, 9, 145-185.

40. D. Ahmed, X. Mao, J. Shi, B. K. Juluri and T. J. Huang, A millisecond micromixer via single-bubble-based acoustic streaming, Lab Chip, 2009, 9, 2738-2741.

41. J.-H. Tsai and L. Lin, Active microfluidic mixer and gas bubble filter driven by thermal bubble micropump, Sens. Actuators, A, 2002, 97-98, 665-671.

42. K. Ryu, J. Zueger, S. K. Chung and S. K. Cho, Underwater propulsion using ACelectrowetting-actuated oscillating bubbles for swimming robots, presented in part at the Proceedings of the 2010 IEEE International Conference on Micro Electro Mechanical Systems (MEMS), Wanchai, Hong Kong, 24-28 January 2010.

43. Y. Zhao and S. K. Cho, Micro air bubble manipulation by electrowetting on dielectric (EWOD): transporting, splitting, merging and eliminating of bubbles, Lab Chip., 2007, 7, 273-280.

44. L. Qiao and C. Luo, Propulsion of a microsubmarine using a thermally oscillatory approach, J. Micromech. Microeng., 2013, 23, 105011.

45. G. T. Gillies, R. C. Ritter, W. C. Broaddus, M. S. Grady, M. A. H. III and R. G. McNeil, Magnetic manipulation instrumentation for medical physics research, Rev. Sci. Instrum., 1994, 65, 533-562.

46. K. Ishiyama, K. I. Arai, M. Sendoh and A. Yamazaki, Spiral-type Micro-machine for Medical Applications, presented in part at the Proceedings of the 2000 International Symposium on Micromechatronics and Human Science, Nagoya, Japan, 22-25 October 2000.

47. R. Dreyfus, J. Baudry, M. L. Roper, M. Fermigier, H. A. Stone and J. Bibette, Microscopic artificial swimmers, Nature, 2005, 437, 862-865.

48. R. G. Cox, Motion of long slender bodies in a viscous fluid. Part I. General theory, $J$. Fluid Mech., 1970, 44, 791-810.

49. E. M. Purcell, The efficiency of propulsion by a rotating flagellum, Proc. Natl. Acad. Sci. USA, 1997, 94, 11307-11311.

50. S. Chattopadhyay, R. Moldovan, C. Yeung and X. L. Wu, Swimming efficiency of bacterium Escherichia coli, Proc. Natl. Acad. Sci. USA, 2006, 103, 13712-13717.

51. L. Zhang, J. J. Abbott, L. Dong, B. E. Kratochvil, D. Bell and B. J. Nelson, Artificial bacterial flagella: Fabrication and magnetic control, Appl. Phys. Lett., 2009, 94, 064107. 
52. D. J. Bell, S. Leutenegger, K. M. Hammar, L. X. Dong and B. J. Nelson, Flagella-Like Propulsion for Microrobots Using a Nanocoil and a Rotating Electromagnetic Field, presented in part at the Proceedings of the 2007 IEEE International Conference on Robotics and Automation, Roma, Italy, 10-14 April 2007.

53. L. Zhang, E. Ruh and D. Grützmacher, Anomalous coiling of $\mathrm{SiGe} / \mathrm{Si}$ and $\mathrm{SiGe} / \mathrm{Si} / \mathrm{Cr}$ helical nanobelts, Nano Lett., 2006, 6, 1311-1317.

54. F. Z. Temel and S. Yesilyurt, Magnetically Actuated Micro Swimming of Bio-Inspired Robots in Mini Channels, presented in part at the Proceedings of the 2011 IEEE Intemational Conference on Mechatronics, Istanbul, Turkey, 13-15 April 2011.

55. W. Gao, S. Sattayasamitsathit, K. M. Manesh, D. Weihs and J. Wang, Magnetically powered flexible metal nanowire motors, J. Am. Chem. Soc., 2010, 132, 14403-14405.

56. S. Floyd, C. Pawashe and M. Sitti, An Untethered Magnetically Actuated Micro-Robot Capable of Motion on Arbitrary Surfaces, presented in part at the Proceedings of the 2008 IEEE International Conference on Robotics and Automation, Pasadena, CA, USA, 19-23 May 2008.

57. H. Lee, A. M. Purdon, V. Chu and R. M. Westervelt, Controlled assembly of magnetic nanoparticles from magnetotactic bacteria using microelectromagnets arrays, Nano Lett., 2004, 4, 995-998.

58. Y. Hiratsuka, M. Miyata, T. Tada and T. Q. P. Uyeda, A microrotary motor powered by bacteria, PNAS, 2006, 103, 13618-13623.

59. M. C. Tarhan, R. Yokokawa, C. Bottier, D. Collard and H. Fujita, A nanoneedle/microtubule composite gliding on a kinesin-coated surface for target molecule transport, Lab Chip., 2010, 10, 86-91.

60. K. Fujimoto, M. Kitamura, M. Yokokawa, I. Kanno, H. Kotera and R. Yokokawa, Colocalization of quantum dots by reactive molecules carried by motor proteins on polarized microtubule arrays, ACS Nano, 2013, 7, 447-455.

61. P. Hanggi and F. Marchesoni, Artificial Brownian motors: Controlling transport on the nanoscale, Rev. Mod. Phys., 2009, 81, 387-442.

62. G. Volpe, I. Buttinoni, D. Vogt, H.-J. Kümmerer and C. Bechinger, Microswimmers in patterned environments, Soft Matter, 2011, 7, 8810-8815.

63. R. Golestanian, T. B. Liverpool and A. Ajdari, Designing phoretic micro- and nanoswimmers, New J. Phys., 2007, 9, 126.

64. H.-R. Jiang, N. Yoshinaga and M. Sano, Active motion of a Janus particle by selfthermophoresis in a defocused laser beam, Phys. Rev. Lett., 2010, 105, 268302. 
65. G. Loget and A. Kuhn, Propulsion of microobjects by dynamic bipolar self-regeneration, J. Am. Chem. Soc., 2010, 132, 15918-15919.

66. Y. Sumino, N. Magome, T. Hamada and K. Yoshikawa, Self-running droplet: Emergence of regular motion from nonequilibrium noise, Phys. Rev. Lett., 2005, 94, 068301.

67. UK Pat., 20081, 1892.

68. A. Jenkins, An elementary treatment of the reverse sprinkler, Am. J. Phys., 2004, 72, 1276-1282.

69. A. Jenkins, Sprinkler head revisited: Momentum, forces, and flows in Machian propulsion, Eur. J. Phys., 2011, 32, 1213-1226.

70. X. M. Chen and A. Prosperetti, Thermal processes in the oscillations of gas bubbles in tubes, J. Acoust. Soc. Am., 1998, 104, 1389-1398.

71. X. Geng, H. Yuan, H. N. Og̃uz and A. Prosperetti, The oscillation of gas bubbles in tubes: Experimental results, J. Acoust. Soc. Am., 1999, 106, 674-681.

72. E. M. Purcell, Life at Low Reynolds Number, American Journal of Physics, 1977, 45, 311.

73. H. N. Og̃uz and A. Prosperetti, The natural frequency of oscillation of gas bubbles in tubes, J. Acoust. Soc. Am., 103, 3301-3308.

74. J. Feng and S. K. Cho, Micro propulsion in liquid by oscillating bubbles, presented in part at the Proceedings of 2013 IEEE 26th International Conference on Micro Electro Mechanical Systems (MEMS), Taipei, 20-24 Jan. 2013.

75. T. Y. Chang, V. G. Yadav, S. D. Leo, A. Mohedas, B. Rajalingam, C.-L. Chen, S. Selvarasah, M. R. Dokmeci and A. Khademhosseini, Cell and protein compatibility of parylene-C surfaces, Langmuir, 2007, 23, 11718-11725.

76. M. P. Norton and D. G. Karczub, Fundamentals of noise and vibration analysis for engineers, Cambridge University Press, New York, USA, 2003.

77. W. L. Nyborg, Acoustic streaming near a boundary, J. Acoust. Soc. Am., 1958, 30, 329339.

78. J. Feng and S. K. Cho, Two-dimensionally steering microswimmer propelled by oscillating bubbles, presented in part at the Proceedings of 2014 IEEE 27th International Conference on Micro Electro Mechanical Systems (MEMS), San Francisco, CA, USA, 26-30 Jan. 2014. 
79. J. Feng and S. K. Cho, Two-dimensionally steering microswimmer propelled by oscillating bubbles, presented in part at the Proceedings of the 2014 IEEE 27th International Conference on Micro Electro Mechanical Systems, San Francisco, US, 2630 January 2014.

80. D. Ahmed, M. Lu, A. Nourhani, P. E. Lammert, Z. Stratton, H. S. Muddana, V. H. Crespi and T. J. Huang, Selectively manipulable acoustic-powered microswimmers, Sci. Rep., 2015, 5, 9741-9748.

81. D. Williams, The Williams Dictionary of Biomaterials, Liverpool University Press, Liverpool, 1999.

82. L. AW, Interfacial bioengineering to enhance surface biocompatibility, Med Device Technol., 2002, 13, 18-21.

83. T. Freier, C. Kunze, C. Nischan, S. Kramer, K. Sternberg, M. sa $\beta$, U. Hopt and K.-P. Schmitz, In vitro and in vivo degradation studies for development of a biodegradable patchbased on poly(3-hydroxybutyrate), Biomaterials, 2002, 23, 2649-2657.

84. B. D. Ulery, L. S. Nair and C. T. Laurencin, Biomedical Applications of Biodegradable Polymers, J Polym Sci B Polym Phys., 2011, 49, 832-864.

85. J. C. Middleton, A. J. Tipton, Synthetic Biodegradable Polymers as Medical Devices, Biomaterials, 2000, 21, 2335-2346.

86. P. C. Beard, A. M. Hurrell and T. N. Mills, Characterization of a Polymer Film Optical Fiber Hydrophone for Use in the Range 1 to $20 \mathrm{MHz}$ : AComparison with PVDF Needle and Membrane Hydrophones, Trans. Ultrason., Ferroelect., Freq. Contr., 2000, 47, 256264.

87. M. R. Steedman, S. L. Tao, H. Klassen and T. A. Desai, Enhanced differentiation of retinal progenitor cells using microfabricated topographical cues, Biomed Microdevices, 2010, 12, 363-369.

88. D. Simon, A. Holland and R. Shanks, Poly(caprolactone) Thin Film Preparation, Morphology, and Surface Texture, J. Appl. Polym. Sci., 2007, 103, 1287-1294.

89. J. Nagstrup, Micro fabrication of biodegradable polymer drug delivery devices, 2013.

90. J. Nagstrup, S. Keller, K. Almdal and A. Boisen, 3D microstructuring of biodegradable polymers, Microelectronic Engineering, 2011, 88, 2342-2344.

91. M. Luo, A. W. Martinez, C. Song, F. Herrault and M. G. Allen, A Microfabricated Wireless RF Pressure Sensor Made Completely of Biodegradable Materials, $J$. Microelectromech. Syst., 2014, 23, 4-13. 
92. C. M. Boutry, R. Kiran, F. Umbrecht and C. Hierold, Processing and quantitative analysis of biodegradable polymers (PLLA and PCL) thermal bonding, J. Micromech. Microeng., 2010, 20, 085006.

93. X.-N. Gu and Y.-F. Zheng, A review on magnesium alloys as biodegradable materials, Front. Mater. Sci. China, 2010, 4, 111-115.

94. F. Witte, N. Hort, C. Vogt, S. Cohen, K. U. Kainer, R. Willumeit and F. Feyerabend, Degradable biomaterials based on magnesium corrosion, Curr. Opin. Solid State Mater. Sci., 2008, 12, 63-72.

95. R. J. P. Williams, Metal Ions in Biological System, Biological Reviews, 2008, 28, 381412.

96. H. G. Seiler, H. Sigel and A. Sigel, Handbook on Toxicity of Inorganic Compounds, Marcel Dekker Inc., New York, 1988.

97. V. D, K. J, S. J and N. P, Mechanical and corrosion properties of newly developed biodegradable Zn-based alloys for bone fixation, Acta Biomaterialia, 2011, 7, 3515-3522.

98. L. Xu, G. Yu, E. Zhang, F. Pan and K. Yang, In vivo corrosion behavior of Mg-Mn-Zn alloy for bone implant application, J Biomed Mater Res A., 2007, 83, 703-711.

99. O. G. Schmidt and K. Eberl, Thin solid films roll up into nanotubes, Nature, 2001, 410, 168.

100. O. G. Schmidt, N. Schmarje, C. Deneke, C. Müller and N.-Y. Jin-Phillipp, ThreeDimensional Nano-objects Evolving from a Two-Dimensional Layer Technology, Adv. Mater., 2001, 13, 756-759.

101. C. Deneke, C. Müller, N. Y. Jin-Phillipp and O. G. Schmidt, Diameter scalability of rolled-up In(Ga)As/GaAs nanotubes, Semicond. Sci. Technol., 2002, 17, 1278-1281.

102. R. Songmuang, C. Deneke and O. G. Schmidt, Rolled-up micro- and nanotubes from single-material thin films, Appl. Phys. Lett., 2006, 89, 223109.

103. Y. Mei, G. Huang, A. A. Solovev, E. B. Ureña, I. Mönch, F. Ding, T. Reindl, R. K. Y. $\mathrm{Fu}$, P. K. Chu and O. G. Schmidt, Versatile Approach for Integrative and Functionalized Tubes by Strain Engineering of Nanomembranes on Polymers, Adv. Mater., 2008, 20, 4085-4090.

104. D. J. Thurmer, C. Deneke, Y. Mei and O. G. Schmidt, Process integration of microtubes for fluidic applications, Appl. Phys. Lett., 2006, 89, 223507.

105. R. Songmuang, A. Rastelli, S. Mendach and O. G. Schmidt, SiOx/Si radial superlattices and microtube optical ring resonators, Appl. Phys. Lett., 2007, 90, 091905. 
106. G. Huang, Y. Mei, D. J. Thurmer, E. Coric and O. G. Schmidt, Rolled-up transparent microtubes as two-dimensionally confined culture scaffolds of individual yeast cells, $L a b$ Chip, 2009, 9, 263-268.

107. A. A. Solovev, Y. Mei, E. B. Ureña, G. Huang and O. G. Schmidt, Small, 2009, 5, 16881692.

108. W. Xi, A. A. Solovev, A. N. Ananth, D. H. Gracias, S. Sanchez and O. G. Schmidt, Rolled-up magnetic microdrillers: towards remotely controlled minimally invasive surgery, Nanoscale, 2013, 5, 1294-1297.

109. K.-N. Lee, Y.-T. Seo, M.-H. Lee, S.-W. Jung, Y.-K. Kim, J.-M. Kim and W. K. Seong, Stress-induced self-rolled metal/insulator bifilm microtube with micromesh walls, $J$. Micromech. Microeng., 2013, 23, 015003.

110. Y. C. Tsui and T. W. Clyne, An analytical model for predicting residual stresses in progressively deposited coatings Part 1: Planar geometry, Thin Solid Films, 1997, 306, 23-33.

111. P. Cendula, S. Kiravittaya, I. Mönch, J. Schumann and O. G. Schmidt, Directional roll-up of nanomembranes mediated by wrinkling, Nano Lett., 2011, 11, 236-240. 